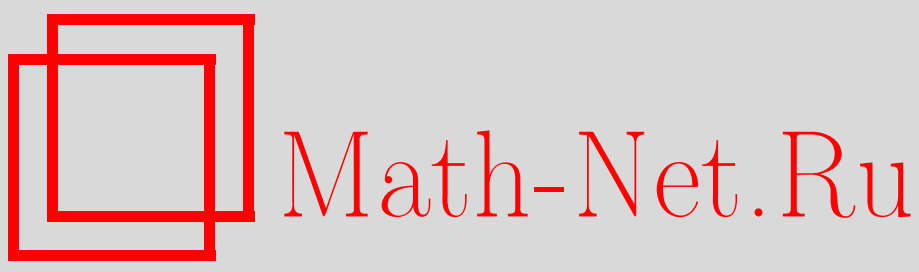

В. 3. Гринес, Е. В. Жужома, Структурно устойчивые диффеоморфизмы с базисными множествами коразмерности один, Изв. РАН. Сер. матем., 2002, том 66, выпуск 2, 3-66

DOI: https://doi.org/10.4213/im378

Использование Общероссийского математического портала Math-Net.Ru подразумевает, что вы прочитали и согласны с пользовательским соглашением http: //www.mathnet.ru/rus/agreement

Параметры загрузки:

IP : 54.174 .149 .18

26 апреля 2023 г., 16:43:08 
УДК $517.9+513.83$

\author{
В.3. Гринес, Е.В. Жужома
}

\title{
Структурно устойчивые диффеоморфизмы с базисными множествами коразмерности один
}

\begin{abstract}
Исследуется топологическая и гомотопическая структура замкнутых $n$-мерных $(n \geqslant 3)$ многообразий, на которых существуют структурно устойчивые диффеоморфизмы с ориентируемыми растягивающимися аттракторами и сжимающимися репеллерами коразмерности один. Полученные результаты применяются для топологической классификации таких диффеоморфизмов на $n$-мерном торе $T^{n}, n \geqslant 3$.

Библиограффия: 69 наименований.
\end{abstract}

\section{Введение}

Структурно устойчивые диффеоморфизмы составляют важный класс динамических систем, и на любом замкнутом многообразии они образуют непустое открытое множество в пространстве диффеоморфизмов. Поэтому представляет интерес задача исследования многообразий, допускаюших структурно устойчивые диффеоморфизмы с дополнительными ограничениями, и задача классификации диффеоморфизмов выделенного класса. Известно, что структурно устойчивые диффеоморфизмы удовлетворяют аксиоме $A$ Смейла [66] (т.е. являются $A$-диффеоморфизмами) и строгому условию трансверсальности [51]. Один из самых замечательных классов структурно устойчивых диффеоморфизмов составляют диффеоморфизмы Аносова [1]. Существует несколько работ, посвяшенных изучению связи между топологией несушего многообразия и динамическими свойствами диффеоморфизмов Аносова коразмерности один. В [39], [55] доказано, что любой диффеоморфизм Аносова коразмерности один на замкнутом многообразии сопряжен гиперболическому автоморфизму коразмерности один тора $T^{n}$. (Напомним, что алгебраический автоморфизм $A: T^{n} \rightarrow T^{n} n$-мерного тора $T^{n}, n \geqslant 3$, называется гиперболическим автоморфизмом коразмерности один, если матрица, определяющая $A$, имеет единственное собственное число, по абсолютной величине либо меньшее, либо большее единицы, а остальные собственные числа лежат соответственно либо вне единичной окружности комплексной плоскости, либо внутри.)

Непосредственным развитием диффеоморфизмов Аносова коразмерности один являются структурно устойчивые диффеоморфизмы с базисными множествами коразмерности один (коразмерность один означает, что топологическая размерность базисного множества на единицу меньше размерности объемлющего многообразия). Поэтому естественно для данных диффеоморфизмов исследовать топологическую структуру несушего многообразия. Если к диффеоморфизму Аносова коразмерности один применить “хирургическую операцию”, то получим структурно

Авторы благодарят РФФИ (гранты № 99-01-00230 и № 02-01-00098) и INTAS (грант 97-1843) за финансовую поддержку.

(C) в.З. Гринес, Е.В. ЖУЖомА, 2002 
устойчивый $A$-диффеоморфизм с нетривиальным базисным множеством коразмерности один, локально гомеоморфным произведению гиперплоскости коразмерности один на канторово множество [66]. В зависимости от исходного диффеоморфизма Аносова, полученное нетривиальное базисное множество $\Omega$ является либо растягиваюшимся аттрактором, либо сжимающимся репеллером. При этом достижимая из $T^{n}-\Omega$ граница любой компоненты множества $T^{n}-\Omega$ состоит из двух инвариантных многообразий коразмерности один, так называемой 2-связки. Следуя [66], будем называть полученный $A$-диффеоморфизм $D A$-диффеоморфизмом.

Согласно [22, теорема 1] произвольное базисное множество коразмерности один является либо аттрактором, либо репеллером. Если оно не образует вложенное компактное подмногообразие коразмерности один, то остается открытым (насколько известно авторам) вопрос, имеются ли другие базисные множества топологической размерности $n-1$, отличные от растягивающихся аттракторов или сжимаюшихся репеллеров (на компактных $n$-мерных многообразиях при $n \geqslant 3$ ). Согласно [22] и [69] растягиваюшиеся аттракторы и сжимающиеся репеллеры коразмерности один локально устроены как произведение канторова множества на гиперплоскость коразмерности один. Априорная возможность существования базисных множеств, которые имеют другую локальную структуру, отмечалась в $[66$, с. 155 , примечание]. В [41] построен структурно устойчивый диффеоморфизм пятимерной сферы с одномерным базисным множеством, которое локально не гомеоморфно произведению канторова множества и интервала. Отметим также примеры базисных множеств, рассмотренных в [47] и [38]. В последней работе на трехмерном замкнутом многообразии построен пример ориентируемого растягиваюшегося аттрактора коразмерности один, который не является расслоением ни над каким многообразием с канторовым множеством в качестве слоя.

В настоящей статье мы рассматриваем базисные множества коразмерности один, которые являются растягиваюшимися аттракторами или сжимающимися репеллерами. Имеется еще одно ограничение, которое мы накладываем на базисное множество, - условие ориентируемости. Грубо говоря, для аттрактора коразмерности один это условие означает, что любое одномерное устойчивое инвариантное многообразие пересекает только "в одну сторону" неустойчивые инвариантные многообразия, из которых состоит базисное множество (точное определение см. в $\S 1)$. Упомянутый вьше $D A$-диффеоморфизм имеет ориентируемое базисное множество коразмерности один, которое является либо растягиваюшимся аттрактором, либо сжимаюшимся репеллером, и такой структурно устойчивый диффеоморфизм может быть построен на торе $T^{n}$ любой размерности $n \geqslant 2$.

Одним из основных результатов статьи в изучении структуры несущего многообразия является теорема 5.1 , которая утверждает, что если $f$ - структурно устойчивый диффеоморфизм замкнутого $n$-мерного многообразия $M^{n}, n \geqslant 3$, и $\Omega$ - ориентируемый растягиваюшийся аттрактор коразмерности один диффеоморфизма $f$, то $M^{n}$ гомотопически эквивалентен $n$-мерному тору $T^{n}$. При этом если $n \neq 4$, то $M^{n}$ гомеоморфен $T^{n}$. Более того, $\Omega$ является единственным нетривиальным базисньг множеством диффеоморфизма $f$ и неблуждающее множество $f$ содержит, по крайней мере, одну источниковую периодическую точку.

Отметим, что теорема 5.1 не справедлива, вообще говоря, для размерности $n=2$. Однако если дополнительно потребовать, чтобы $\Omega$ содержало только связки степени 2 и $M^{2}-\Omega$ состояло из односвязных компонент, то эта теорема будет справедлива и в случае $n=2$. 
Из теоремы 5.1 непосредственно следует, что если $f$ - структурно устойчивый диффеоморфизм $n$-мерного замкнутого многообразия $M^{n}, n \geqslant 3$, неблуждающее множество которого содержит ориентируемый растягивающийся аттрактор $\Omega$ коразмерности один, то спектральное разложение диффеоморфизма $f$ состоит из $\Omega$, конечного (возможно, пустого) множества периодических орбит индекса $n-1$ и конечного непустого множества периодических орбит индекса $n$.

После этого результата естественно возникает задача топологической классификации структурно устойчивых диффеоморфизмов с ориентируемыми базисными множествами коразмерности один на $n$-мерном торе $T^{n}, n \geqslant 3$. Отметим, что процесс классификации можно условно разделить на три этапа. Сперва строится полный топологический инвариант, определяющий классы топологической сопряженности, затем выделяются допустимые инварианты и, наконец, для данного допустимого инварианта строится стандартньй представитель (т.е. диффеоморфизм с данным инвариантом).

В настоящей статье для структурно устойчивого диффеоморфизма $f$ тора $T^{n}$, $n \geqslant 3$, с ориентируемым растягивающимся аттрактором коразмерности один строится полный инвариант топологической сопряженности, который представляет собой конечный набор периодических орбит некоторого гиперболического автоморфизма $A(f)$ коразмерности один, при этом каждой периодической орбите приписано некоторое натуральное число (см. подробности в $\S 6$ ). Этот инвариант мы называем допустимой $d$-сигнатурой. Любой конечный набор периодических орбит с приписанным каждой из этих орбит произвольным натуральным числом образует допустимую $d$-сигнатуру $\left\{O_{j}, d_{j}\right\}_{j=1}^{r}$. Основные результаты статьи по классификации составляют теоремы 6.2 и 6.3. Теорема 6.2 устанавливает необходимые и достаточные условия топологической сопряженности двух диффеоморфизмов из рассматриваемого класса, а теорема 6.3 доказывает существование представителя класса топологической сопряженности для каждого набора топологических инвариантов.

Отметим некоторые результаты, относящиеся к рассматриваемой тематике, и прокомментируем полученные. Без предположения о структурной устойчивости Плыкин [25], [26] доказал, что устойчивое многообразие ориентируемого растягивающегося $C$-плотного аттрактора коразмерности один имеет гомотопический тип тора $T^{n}$, из которого выколото конечное число точек (см. также [17], где получены близкие результаты). В частности, такой аттрактор имеет окрестность указанного вида. Первое утверждение теоремы 5.1 говорит о том, что при наличии структурной устойчивости ориентируемый растягивающийся аттрактор коразмерности один является $C$-плотным, и гомотопическую эквивалентность тору $T^{n}$ можно доказать для всего объемлюшего многообразия. Заметим, что без предположения о структурной устойчивости первое утверждение теоремы 5.1 неверно. Аналогично [64] (см. также [40] для $n=3$ ) можно построить структурно неустойчивый $A$-диффеоморфизм $f$ многообразия, гомеоморфного связной сумме двух торов $T^{n}, n \geqslant 3$, с неблуждаюшим множеством, состоящим из одного ориентируемого растягивающегося аттрактора коразмерности один и одного ориентируемого сжимаюшегося репеллера коразмерности один. Отметим, что первое утверждение теоремы 5.1 для размерности $n=\operatorname{dim} M=2$ неверно даже в случае структурной устойчивости [10].

Что касается второго утверждения теоремы 5.1 о единственности нетривиального базисного множества, то из него следует, что если $A$-диффеоморфизм $f$ имеет, 
по крайней мере, два нетривиальных базисных множества, одно из которых - либо ориентируемый растягивающийся аттрактор, либо ориентируемый сжимающийся репеллер коразмерности один, то диффеоморфизм $f$ структурно неустойчив.

Упомянутый пример Робинсона-Вильямса [64] показывает, что второе утверждение теоремы 5.1 также неверно без предположения о структурной устойчивости. То же самое замечание относится и к третьему утверждению теоремы 5.1 о сушествовании тривиальных аттракторов или репеллеров (последние часто называются источниковой или стоковой периодической орбитой). Отметим несколько результатов для размерности $n=\operatorname{dim} M=2$, относящиеся к третьему утверждению теоремы 5.1. Для двумерной сферы и двумерного тора это утверждение справедливо и в случае, когда диффеоморфизм $f$ не является структурно устойчивым, а лиш удовлетворяет аксиоме $A$ Смейла, а базисное множество может быть неориентируемым. В случае сферы это непосредственно следует из результата Плыкина [23], доказавшего, что если на многообразии $M^{2}$ сушествует гомотопная нулю простая замкнутая кривая, составленная из отрезков устойчивого и неустойчивого многообразий какой-либо точки, принадлежащей одномерному аттрактору (репеллеру), то в диске, ограниченном этой кривой, необходимо имеется источниковая (стоковая) периодическая точка диффеоморфизма $f$. Для двумерного тора аналогичное утверждение следует из [9, теорема 2.1] и из того, что неблуждающее множество $A$-диффеоморфизма двумерного тора может содержать не более одного просторно расположенного одномерного базисного множества [23]. Аналогичные утверждения имеют место для проективной плоскости и бутылки Клейна [24]. В работе [10] доказано, что третье утверждение справедливо на замкнутых ориентируемых поверхностях любого рода.

Перейдем к вопросам топологической классификации. Возможность топологической классификации структурно устойчивых диффеоморфизмов с ориентируемыми базисными множествами коразмерности один основана на установленной в настоящей работе структуре неблуждаюшего множества таких диффеоморфизмов (следствие 5.1) и классификации $A$-диффеоморфизмов тора $T^{n}, n \geqslant 3$, с ориентируемыми растягивающимися аттракторами коразмерности один, полученной авторами в [11], [12].

Остановимся подробнее на результатах по классификации $A$-диффеоморфизмов. Если неблуждаюшее множество $A$-диффеоморфизма содержит нетривиальные базисные множества коразмерности один, то при решении задачи топологической классификации таких диффеоморфизмов естественно вначале возникает задача топологической сопряженности ограничений диффеоморфизмов на базисные множества, которая формулируется следующим образом. Пусть $\Omega, \Omega^{\prime}$ - базисные множества коразмерности один $A$-диффеоморфизмов $f, f^{\prime}$ соответственно. Требуется найти необходимые и достаточные условия существования гомеоморфизма $g: M \rightarrow M$ такого, что

$$
g(\Omega)=\Omega^{\prime} \quad \text { и }\left.f^{\prime}\right|_{\Omega}=\left.g \circ f \circ g^{-1}\right|_{\Omega^{\prime}} .
$$

Первые результаты в решении этой задачи были получены первым автором статьи для размерности $n=\operatorname{dim} M=2$ для ориентируемых одномерных аттракторов [7]-[9], и затем были обобщены Плыкиным на случай просторно расположенных аттракторов [26] (нетривиальное базисное множество $\Omega$ называется просторно расположенным, если не сушествует гомотопной нулю петли, образованной парой отрезков устойчивого и неустойчивого многообразия какой-либо точки из $\Omega$ ) 
и для размерности $n=\operatorname{dim} M \geqslant 3$ на случай аттракторов со связками степени не больше двух [25], [26]. Поставленная задача для размерности $n=\operatorname{dim} M=2$ была полностью решена Гринесом и Калаем для произвольных аттракторов на ориентируемой поверхности рода $p \geqslant 0$ [13]-[15]. Отметим также работу Бонатти и Ланжевена [34], где получена классификация произвольных структурно устойчивых диффеоморфизмов ориентируемых компактных поверхностей рода $p \geqslant 0$.

Имеется цикл работ Жирова [16], посвященных комбинаторному описанию и конечной алгоритмической классификации одномерных аттракторов на поверхностях.

Структура статьи следуюшая. В $\S 1$ даются основные определения. В $\S 2$ исследуется топологическая структура многообразия в предположении, что характеристические сферы ориентируемого растягивающегося аттрактора коразмерности один ограничивают в многообразии шар (в дальнейшем показывается, что это условие выполняется, если диффеоморфизм структурно устойчивый). В 33 исследуется топологическая структура универсальной накрывающей и доказывается теорема 3.1 , которая является ключевой в доказательстве первого утверждения теоремы 5.1. В $\S 4$ доказывается теорема 4.2, которая является ключевой в доказательстве остальных утверждений теоремы 5.1. В некотором смысле ее можно рассматривать как одно достаточное условие структурной неустойчивости. Теорема 5.1 доказывается в $\S 5$. Классификационные теоремы 6.2 и 6.3 доказываются в $\oint 6$.

Авторы благодарят Д. В. Аносова, С. Х. Арансона, М. И. Малкина, В. С. Медведева, Р. В. Плькина, Х. Бонатти, Р. Ланжевена, П. Швейцера, Р. Вильямса за плодотворные обсуждения.

\section{§1. Основные определения и вспомогательные предложения}

Напомним некоторые понятия и факты, касаюшиеся диффеоморфизмов, удовлетворяюших аксиоме $A$ Смейла [66]. Хорошим источником являются книги [48], [19], а также обзорные статьи [4], [5], [27] и [66]. Везде далее под диффеоморфизмом понимается $C^{\infty}$-диффеоморфизм.

1.1. $A$-диффеоморфизмы. Пусть $f$-диффеоморфизм замкнутого $n$-мерного, $n \geqslant 2$, многообразия $M=M^{n}$, снабженного некоторой римановой метрикой $d$. Множество $\Lambda \subset M$, инвариантное относительно $f$, называется гиперболическим, если ограничение $T_{\Lambda} M$ касательного расслоения $T M$ многообразия $M$ на $\Lambda$ можно представить в виде суммы Уитни $E_{\Lambda}^{s} \oplus E_{\Lambda}^{u} d f$-инвариантных подрасслоений $E_{\Lambda}^{s}$, $E_{\Lambda}^{u}, \operatorname{dim} E_{x}^{s}+\operatorname{dim} E_{x}^{u}=n, x \in \Lambda$, и существуют константы $C_{s}>0, C_{u}>0$, $0<\lambda<1$ такие, что

$$
\begin{aligned}
\left\|d f^{n}(v)\right\| \leqslant C_{s} \lambda^{n}\|v\|, & v \in E_{\Lambda}^{s}, \quad n>0 \\
\left\|d f^{-n}(v)\right\| \leqslant C_{u} \lambda^{n}\|v\|, & v \in E_{\Lambda}^{u}, \quad n>0 .
\end{aligned}
$$

Точка $x \in M$ назьвается неблуждающей, если для любой ее окрестности $U(x)$ и любого натурального числа $N$ найдется $n_{0} \in \mathbb{Z},\left|n_{0}\right| \geqslant N$, такое, что $f^{n_{0}}(x) \in U(x)$. Множество неблуждающих точек диффеоморфизма $f$ будем обозначать через $N W(f)$. Диффеоморфизм $f$ удовлетворяет аксиоме $A$ (или, что то же самое, является $A$-диффеоморфизмом), если множество $N W(f)$ гиперболическое и периодические точки всюду плотны в $N W(f)$. 
1.2. Устойчивые и неустойчивые многообразия. Гиперболическая структура на неблуждающем множестве порождает сушествование так называемых устойчивых и неустойчивых многообразий. Их существование и свойства исследовались во многих работах (см., например, [1], [19], [44]-[66]), начиная с работ Адамара [42] и Перрона [57], [58]. Поэтому соответствующие результаты иногда называют теоремой Адамара-Перрона.

Пусть $\Lambda$ - компактное гиперболическое множество диффеоморфизма $f: M \rightarrow$ $M$. Тогда для любого $x \in \Lambda$ сушествует инъективная $C^{\infty}$-иммерсия $J_{x}^{s}: \mathbb{R}^{s} \rightarrow M$, образ которой $J_{x}^{s}\left(\mathbb{R}^{s}\right) \stackrel{\text { def }}{=} W^{s}(x)$ называется устойчивым многообразием точ$\kappa u x$, такая, что вьполняются следуюшие свойства:

1) точки $x, y \in M$ принадлежат одному многообразию $W^{s}(x)$ тогда и только тогда, когда $d\left(f^{n}(x), f^{n}(y)\right) \rightarrow 0$ при $n \rightarrow+\infty$;

2) $f\left(W^{s}(x)\right)=W^{s}(f(x)), T_{x} W^{s}(x)=E_{\Lambda}^{s}$;

3) если $x, y \in \Lambda$, то либо $W^{s}(x)=W^{s}(y)$, либо $W^{s}(x) \cap W^{s}(y)=\varnothing$;

4) если точки $x, y \in \Lambda$ близки на $M$, то $W^{s}(x), W^{s}(y)$ близки на компактных множествах (это свойство обычно называют теоремой о непрерывной зависимости устойчивых многообразий от начальных условий).

Неустойчивое многообразие $W^{u}(x)$ точки $x \in \Lambda$ определяется как устойчивое многообразие относительно диффеоморфизма $f^{-1}$. Неустойчивые многообразия обладают аналогичными свойствами. Учитывая свойство 2), устойчивые и неустойчивые многообразия называют инвариантнымми многообразиями. Отметим, что с точки зрения дифференциальной топологии инвариантные многообразия не являются "настояшими" подмногообразиями. Однако они являются иммерсированными подмногообразиями (см., например, [63]).

Обозначим через $W_{\varepsilon}^{s(u)}(x) \varepsilon$-окрестность точки $x$ в многообразии $W^{s(u)}(x)$ (в его внутренней топологии). Следующее утверждение, доказанное Смейлом [66], часто называют теоремой о локальной структуре произведения: пусть диффеоморфизм $f$ удовлетворяет аксиоме $A$; тогда существует $\varepsilon>0$ такое, что для любых $\varepsilon$-близких точек $x_{1}, x_{2} \in N W(f)$ пересечение $W_{\varepsilon}^{s}\left(x_{1}\right) \cap W_{\varepsilon}^{u}\left(x_{2}\right)$ состоит из единственной точки, принадлежащей $N W(f)$.

1.3. Спектральное разложение. Смейл [66] доказал следуюшее утверждение, известное как теорема о спектральном разложении: пусть диффеоморфизм $f$ компактного многообразия $M$ удовлетворяет аксиоме $A$; тогда множество неблуждающих точек $N W(f)$ представляется в виде конечного объединения попарно непересекающихся замкнутых инвариантных множеств $\Omega_{1}, \ldots, \Omega_{k}$, называемых базисными множествами, каждое из которых содержит всюду плотную орбиту. При этом многообразие $M$ можно представить в виде

$$
M=\bigcup_{i=1}^{k} W^{s}\left(\Omega_{i}\right)=\bigcup_{i=1}^{k} W^{u}\left(\Omega_{i}\right), \quad W^{s(u)}\left(\Omega_{i}\right)=\bigcup_{x \in \Omega_{i}} W^{s(u)}(x) .
$$

Базисное множество называется нетривиальным. если оно не является периодической орбитой (в частности, не является неподвижной точкой).

В силу транзитивности $f$ на каждом базисном множестве $\Omega_{i}$ слои расслоений $E_{\Omega_{i}}^{s}, E_{\Omega_{i}}^{u}$ имеют постоянную размерность во всех точках $x \in \Omega_{i}$. Индексом (Морса) базисного множества $\Omega_{i}$ называется размерность $\operatorname{dim} E_{x}^{u}$, где $x$ - любая точка из $\Omega_{i}$. 
Базисное множество $\Omega$ называется аттрактором, если существует окрестность $U$ этого множества такая, что $\bigcap_{j \geqslant 0} f^{j}(U)=\Omega$. Множество называется $p e-$ пеллером, если оно является аттрактором для $f^{-1}$. Ясно, что многие результаты об аттракторах можно перефразировать применительно к репеллерам, и наоборот.

Из [2] и [35] вытекает следуюшее уточнение структуры базисного множества. Каждое базисное множество $\Omega_{i}$ представляется в виде конечного объединения непересекающихся компактов $\Omega_{i 1}, \ldots, \Omega_{i h}$, которые под действием $f$ циклически переходят друг в друга. Более того, устойчивое и неустойчивое многообразие любой точки $x \in \Omega_{i j}$ содержит множество, плотное в $\Omega_{i j}$. Каждое $\Omega_{i j}$ называется $C$-плотной компонентой базисного множества $\Omega_{i}$. Базисное множество называется $C$-nлотныцм, если оно имеет ровно одну $C$-плотную компоненту, с которой оно совпадает.

1.4. Структурно устойчивые диффеоморфизмы. Пусть $\operatorname{Diff}^{1}(M)$ - пространство $C^{1}$-диффеоморфизмов многообразия $M$, наделенное равномерной $C^{1}$-топологией. Два диффеоморфизма $f, g \in \operatorname{Diff}^{1}(M)$ называются (топологически) coпряжсенны.ми, если сушествует гомеоморфизм $\varphi: M \rightarrow M$ такой, что $\varphi \circ f=g \circ \varphi$. Диффеоморфизм $f \in \operatorname{Diff}^{-1}(M)$ называется структурно устойчивым, если существует его окрестность $U(f) \subset \operatorname{Diff}^{1}(M)$ такая, что любой диффеоморфизм $g \in U(f)$ сопряжен $f$. Если потребовать, чтобы сопрягаюший гомеоморфизм был близок к тождественному в $C^{0}$-топологии, то получим определение грубого диффеоморфизма (см. обзор [3], где обсуждаются различные определения и соответствующие результаты).

При формулировке условий структурной устойчивости большую роль играет условие, которое называют сильным условием трансверсальности. Пусть $W_{1}, W_{2} \subset M$ - два иммерсированных подмногообразия, имеющие непустое пересечение. По определению $W_{1}, W_{2}$ пересекаются трансверсально, если для любой точки $x \in W_{1} \cap W_{2}$ касательное пространство $T_{x} M$ порождается касательными подпространствами $T_{x} W_{1}$ и $T_{x} W_{2}$. В частности, если $W_{1}, W_{2}$ пересекаются трансверсально, то $\operatorname{dim} T_{x} W_{1}+\operatorname{dim} T_{x} W_{2} \geqslant \operatorname{dim} T_{x} M$.

Говорят, что $A$-диффеоморфизм удовлетворяет сильному условию трансверсальности, если для любых точек $x, y \in N W(f)$ многообразия $W^{s}(x), W^{u}(y)$ имеют только трансверсальные пересечения.

Известно [51], [62], что диффеоморфизм структурно устойчив тогда и только тогда, когда он является $A$-диффеоморфизмом и удовлетворяет сильному условию трансверсальности. Необходимость доказал Мане [51], достаточность - Робин$\mathrm{coH}[62]$.

1.5. Базисные множества коразмерности один. Под размерностью базисного множества мы понимаем топологическую размерность. Аттрактор $\Omega$ называется растягивающимся, если размерность $\Omega$ совпадает с размерностью неустойчивого многообразия любой его точки. Базисное множество диффеоморфизма $f$ называется сжимающимся репеллером, если оно является растягивающимся аттрактором диффеоморфизма $f^{-1}$.

Если $\operatorname{dim} \Omega=n-1$, то $\Omega$ называется базисным множеством коразмерносmи один. Согласно [22, теорема 1] базисное множество $\Omega$ коразмерности один является либо аттрактором, либо репеллером. В этом случае неустойчивое (если $\Omega$ - аттрактор), либо устойчивое (если $\Omega$ - репеллер) многообразие любой точки $x \in \Omega$ принадлежит $\Omega$. Согласно [22, теорема 2] растягиваюшийся аттрак- 
тор или сжимающийся репеллер коразмерности один имеет локальную структуру прямого произведения $(n-1)$-мерного евклидова пространства и канторова множества. В этом случае $\Omega$ состоит либо из неустойчивых (если $\Omega$ - аттрактор), либо из устойчивых (если $\Omega$ - репеллер) $(n-1)$-мерных многообразий своих точек. Обратное, очевидно, также справедливо, т.е. если базисное множество $\Omega$ состоит либо из неустойчивых (если $\Omega$ - аттрактор), либо из устойчивых (если $\Omega$ - репеллер) $(n-1)$-мерных многообразий своих точек и имеет вышеуказанную локальную структуру прямого произведения, то $\Omega$ есть либо растягивающийся аттрактор, либо сжимающийся репеллер коразмерности один. Отметим, что до настоящего времени (насколько нам известно) не доказана достаточно правдоподобная гипотеза о том, что аттрактор (соответственно, репеллер) коразмерности один, не образуюший компактного подмногообразия, является растягиваюшимся аттрактором (соответственно, сжимаюшимся репеллером).

Каждое инвариантное многообразие $W^{s}(x), W^{u}(x)$ есть образ евклидового пространства относительно гладкой иммерсии (т.е. $W^{s}(x), W^{u}(x)$ являются иммерсированными подмногообразиями). Поэтому открытые диски $W_{\alpha}^{s}(x), W_{\beta}^{u}(x)$ являются ориентируемыми и нормально ориентируемыми иммерсированными подмногообразиями дополнительной размерности, $\operatorname{dim} W_{\alpha}^{s}(x)+\operatorname{dim} W_{\beta}^{u}(x)=n$, для любых $\alpha>0, \beta>0$. Следовательно, корректно определение ориентируемости (по Гринесу) базисного множества. Будем говорить, что базисное множество $\Omega$ ориентируемо, если для любой точки $x \in \Omega$ и любых фиксированных чисел $\alpha>0$, $\beta>0$ индекс пересечения $W_{\alpha}^{s}(x) \cap W_{\beta}^{u}(x)$ во всех точках пересечения один и тот же $(+1$, либо -1$)[7],[8]$. В противном случае базисное множество $\Omega$ называется неориентируемым.

Отметим, что в настоящей статье и, в частности, в приведенном определении ориентируемости базисного множества, несущее многообразие $M^{n}$ может быть как ориентируемым, так и неориентируемым (об индексе пересечения подмногообразий в неориентируемом многообразии см. [32, с. 175]).

1.6. Граничные периодические точки. Пусть $\Omega$ - базисное множество индекса (Морса) $n-1 A$-диффеоморфизма $f: M \rightarrow M$. Следовательно,

$$
\operatorname{dim} E_{\Omega}^{s} \stackrel{\text { def }}{=} \operatorname{dim} E_{x}^{s}=1 \text { для любой точки } \quad x \in \Omega .
$$

Обозначим через $(x, y)^{s}$ (соответственно, $[x, y]^{s}$ ) открытую (соответственно, замкнутую) дугу на одномерном устойчивом многообразии $W^{s}(z)$ с конщевыми точками $x, y \in W^{s}(z)$, где $z \in \Omega$.

ПРЕДЛОЖЕНИЕ 1.1. Пусть $\Omega$ - базисное множество индекса $n-1 A$-диффеоморфизма $f: M^{n} \rightarrow M^{n}, \quad n \geqslant 2$. Предположим, что $x \in \Omega$ - непериодическая точка. Тогда обе компоненты множества $W^{s}(x)-x$ пересекают $W^{u}(\Omega)$. Более того, если $W^{u}(x)$ не содержит периодических точек, то обе компоненты множества $W_{\delta}^{s}(x)-x$ пересекают $W^{u}(\Omega)$ для любого числа $\delta>0$.

ДокАЗАТЕльство. Пусть $f^{n_{i}}(x) \rightarrow x_{*}$ - сходящаяся последовательность при $n_{i} \rightarrow-\infty$. Так как $\Omega$ - замкнутое и инвариантное множество, то $x_{*} \in \Omega$. Вначале предположим, что $W^{u}(x)$ не содержит периодических точек. Тогда, перейдя, если необходимо, к подпоследовательности, можем считать, что $W^{u}\left(f^{n_{i}}(x)\right)=$ 


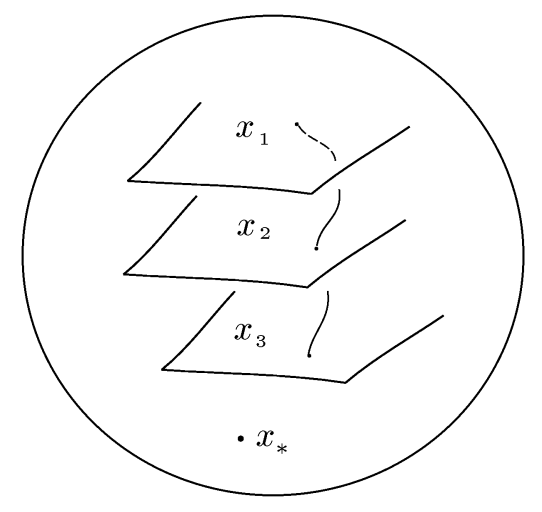

Рис. 1. Окрестность точки $x_{*}$

$f^{n_{i}}\left(W^{u}(x)\right)$ попарно не пересекаются. Из локальной структуры произведения следует, что $W_{\varepsilon}^{s}\left(x_{2}\right) \cap W_{\varepsilon}^{u}\left(x_{j}\right), j=1,3$, есть одна точка множества $\Omega$ из некоторой окрестности точки $x_{*}$, где $x_{i}=f^{n_{i}}(x)$ (см. рис. 1). Не уменьшая обшности, можем считать, что точки $W_{\varepsilon}^{s}\left(x_{2}\right) \cap W_{\varepsilon}^{u}\left(x_{1}\right), W_{\varepsilon}^{s}\left(x_{2}\right) \cap W_{\varepsilon}^{u}\left(x_{3}\right)$ принадлежат различным компонентам множества $W_{\varepsilon}^{s}\left(x_{2}\right)-x_{2}$. Следовательно, обе компоненты множества $W^{s}(x)-x$ пересекают $\Omega$. Более того, для некоторой подпоследовательности $f^{n_{j}}(x) \rightarrow x_{*}$ обе компоненты множества $W_{\varepsilon}^{s}\left(f^{n_{j}}(x)\right)-f^{n_{j}}(x)$ пересекают $W^{u}(\Omega)$. Так как

$$
\lim _{n_{j} \rightarrow-\infty} \operatorname{diam} f^{-n_{j}}\left(W_{\varepsilon}^{s}\left(f^{n_{j}}(x)\right)\right) \rightarrow 0,
$$

то обе компоненты множества $W_{\delta}^{s}(x)-x$ пересекают $W^{u}(\Omega)$ для любого $\delta>0$.

Предположим теперь, что $W^{u}(x)$ содержит периодическую точку $x_{0} \in \Omega$. Для простоты будем считать $x_{0}$ неподвижной точкой. Так как ограничение $\left.f\right|_{W^{u}\left(x_{0}\right)}$ растягивает инвариантное многообразие $W^{u}\left(x_{0}\right)$, то существует последовательность $n_{i} \rightarrow+\infty$ такая, что расстояние между $f^{n_{i}}(x)$ и $f^{n_{j}}(x)$ в метрике многообразия $W^{u}(x)$ больше, чем $\varepsilon$, при $i \neq j$. Перейдя, если необходимо, к подпоследовательности, можно считать, что $f^{n_{i}}(x)$ сходится к некоторой точке $x_{*} \in \Omega$. Дальнейшее рассуждение полностью аналогично рассмотренному выше.

ПРЕДЛОЖЕНИЕ 1.2. Пусть $\Omega$ - растягивающийся аттрактор коразмерности один (ориентируемый или неориентируемый) A-диффеоморфизма $f$ : $M^{n} \rightarrow M^{n}, \quad n \geqslant 2$. Предположим, что $x \in \Omega$-непериодическая точка. Тогда обе компоненты множества $W^{s}(x)-x$ пересекают $\Omega$. Более того, если $W^{u}(x)$ не содержит периодических точек, то обе компоненты множества $W_{\delta}^{s}(x)-x$ пересекают $\Omega$ для любого числа $\delta>0$.

ДокАЗАТЕЛЬСТво вытекает из предложения 1.1, поскольку для растягивающегося аттрактора $\Omega$ выполняется равенство $W^{u}(\Omega)=\Omega$.

Везде далее в этом параграфе $\Omega$ - растягивающийся аттрактор коразмерности один. Следуя [7], [8], будем называть точку $p \in \Omega$ граничной, если одна из компонент множества $W^{s}(p)-p$ не пересекается с $\Omega$. Так как $\Omega \neq M$, то граничные точки существуют [7], [8], [23], [55]. Из работ [7], [8], [23], [26], [55] вытекают следующие свойства граничных точек, которые для ссылок мы сформулируем в виде леммы. 
ЛЕмма 1.1. Пусть $\Omega$-растягивающийся аттрактор коразмерности один. Tогда:

1) в $\Omega$ имеется только конечное ненулевое число граничных точек и все они периодические;

2) для каждой граничной точки $p \in \Omega$ имеется только одна компонента множества $W^{s}(p)-p$, которая не пересекается $c \Omega$, эту компоненту мы будем обозначать через $W_{\varnothing}^{s}(p)$;

3) если $p \in \Omega$ - граничная точка, то для любой точки $x \in W^{u}(p)-p$ существует единственная дуга $(x, y)^{s} \stackrel{\text { def }}{=}(x, y)_{\varnothing}^{s}$ такая, ито $(x, y)^{s} \cap \Omega=\varnothing$ $u \in \in$.

В следующих предложениях детализируется структура устойчивого многообразия растягивающегося аттрактора коразмерности один.

ПРЕДЛОЖЕНИЕ 1.3. Пусть $\Omega-C$-плотный растягивающийся аттрактор коразмерности один (ориентируемый или неориентируемый) $A$-диффеоморфизма $f: M^{n} \rightarrow M^{n}, \quad n \geqslant 2$. Предположим, что для некоторой точки $x \in \Omega$ одна из компонент множества $W^{s}(x)-x$, которую мы обозначим через $W^{s+}(x)$, содержит последовательность точек $x_{k} \in W^{s+}(x) \cap \Omega$, не ограниченную в топологии кривой $W^{s+}(x)$. Тогда пересечение $W^{s+}(x) \cap \Omega$ всюду плотно в $\Omega$. В частности, если $W^{s}(x)$ не содерэст граничных периодических точек, то пересечения $W^{s+}(x) \cap \Omega$ и $W^{s-}(x) \cap \Omega$ всюду плотны в $\Omega$, где $W^{s \pm}(x)$ - компоненты множества $W^{s}(x)-x$.

ДокАЗАТЕЛЬство. Перейдя, если необходимо, к подпоследовательности, можно считать, что последовательность точек $x_{k} \in W^{s+}(x) \cap \Omega$ сходится. Так как множество $\Omega$ замкнуто, то $x_{k} \rightarrow z \in \Omega$ при $k \rightarrow \infty$ для некоторой точки $z$. Сперва предположим, что $z \in \Omega-W^{s}(x)$. Возьмем любую точку $y \in \Omega$ и любую $\eta$-окрестность $U_{\eta}(y)$ точки $y, \eta>0$. Так как $\Omega-C$-плотное, то существует дуга $\left[z, z_{\eta}\right]^{s}$ с $z_{\eta} \in U_{\eta}(y)$ (см. рис. 2). В силу теоремы о непрерывной зависимости устойчивых инвариантных многообразий от начальных условий существует $\delta>0$ такое, что каждое устойчивое многообразие $W^{s}\left(x_{k}\right)$, проходящее через $\delta$-окрестность $U_{\delta}(z)$ точки $z$, пересекает $U_{\eta}(y)$, и имеется точка $y_{k}$ с $\left[x_{k}, y_{k}\right]^{s} \subset U_{\delta}\left(\left[z, z_{\eta}\right]^{s}\right)$, где $U_{\delta}\left(\left[z, z_{\eta}\right]^{s}\right)-\delta$-окрестность дуги $\left[z, z_{\eta}\right]^{s}$. Возьмем $\delta>0$ такое, что $x \notin U_{\delta}\left(\left[z, z_{\eta}\right]^{s}\right)$. Тогда $x \notin\left[x_{k}, y_{k}\right]^{s}$ и, следовательно, $W^{s+}(x) \cap$ $U_{\eta}(y) \neq \varnothing$. Поэтому $W^{s+}(x) \cap \Omega$ плотно в $\Omega$.

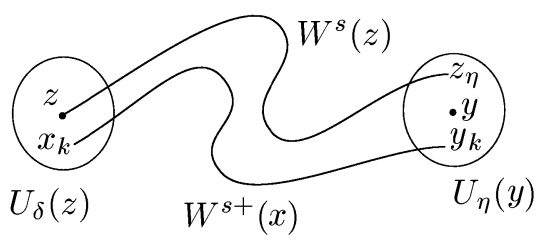

Рис. 2. Дуга $\left[z, z_{\eta}\right]^{s}$

Осталось рассмотреть случай $z \in \Omega \cap W^{s}(x)$. В силу теоремы о непрерьвной зависимости инвариантных многообразий от начальных условий и локальной структуры произведения отсюда вытекает, что $W^{s+}(x) \cap \Omega$ плотно в $W^{s}(x) \cap \Omega$. 
В силу $C$-плотности $\Omega$ множество $W^{s}(x) \cap \Omega$ плотно в $\Omega$. Поэтому $W^{s+}(x) \cap \Omega$ также плотно в $\Omega$.

Если $W^{s}(x)$ не содержит граничных периодических точек, то каждая компонента $W^{s \pm}(x)$ множества $W^{s}(x)-x$ содержит последовательность точек $x_{k} \in$ $W^{s \pm}(x) \cap \Omega$, не ограниченную в топологии кривой $W^{s \pm}(x)$. Из доказанного вьше следует, что $W^{s+}(x) \cap \Omega$ и $W^{s-}(x) \cap \Omega$ всюду плотны в $\Omega$.

1.7. Связки базисного множества коразмерности один. Пусть $G \subset M-$ открытое множество с границей $\partial G$ в смысле теоретико-множественной топологии, $\partial G=\cos G-\operatorname{int} G$. Подмножество $\delta G \subset \partial G$ называется достижимой из $G$ границей, если для любой точки $x \in \delta G$ найдется открытая дуга, полностью лежашая в $G$ и такая, что $x$ является одной из ее конщевых точек. Иногда будем называть $\delta G$ достижимой изнутри границей области $G$.

Пусть $\Omega$ - растягиваюшийся аттрактор коразмерности один $A$-диффеоморфизма $f: M \rightarrow M$. В работах [7], [8], [23], [26] была исследована достижимая изнутри гранища открытого множества $M-\Omega: \delta(M-\Omega)$ есть объединение конечного числа неустойчивых многообразий всех граничных периодических точек аттрактора $\Omega$. Эта достижимая гранища разбивается на попарно непересекающиеся связки, которые определяются следуюшим образом: попарно различные неустойчивые многообразия $W^{u}\left(p_{1}\right), \ldots, W^{u}\left(p_{k}\right)$ образуют $k$-связку, если существуют точки $x_{i} \in W^{u}\left(p_{i}\right)$ и дуги $\left(x_{i}, y_{i}\right)_{\varnothing}^{s}, y_{i} \in W^{u}\left(p_{i+1}\right), 1 \leqslant i \leqslant k+1$, где мы считаем $W^{u}\left(p_{k+1}\right)=W^{u}\left(p_{1}\right)$ (таким образом, $y_{k} \in W^{u}\left(p_{1}\right)$ ), при этом число $k$ максимально возможное (другими словами, не сушествует $(k+1)$-связки, которая содержит данную связку). Точки $p_{1}, \ldots, p_{k}$ называются ассоциированными (см. рис. 3 ).

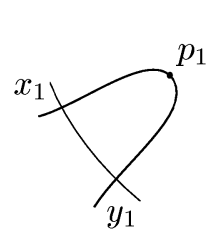

$a$

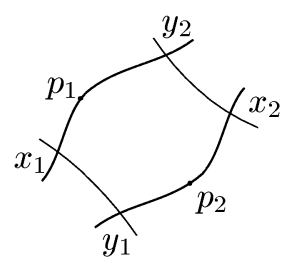

$b$

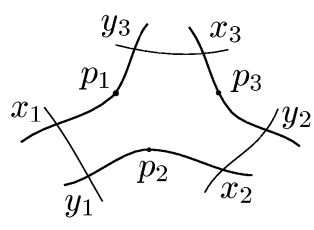

$c$

Рис. 3. $a$-1-связка, $b-2$-связка, $c-3$-связка

Плькин [26] доказал, что в размерности $n=\operatorname{dim} M \geqslant 3$ возможны только 1- и 2-связки. Нетрудно видеть, что у ориентируемого растягивающегося аттрактора не может быть 1-связок. Таким образом, граничные периодические точки растягиваюшегося ориентируемого аттрактора $\Omega$ распадаются на пары ассоциированных точек, которые мы будем обозначать через $\left\{p_{i}, q_{i}\right\}$, иногда опуская индекс.

ПРЕДЛОЖЕНИЕ 1.4. Пусть $\Omega$ - растягивающийся аттрактор (ориентируемый или неориентируемый) $A$-диффеоморфизма $f: M^{n} \rightarrow M^{n}, n \geqslant 2$. Если точка z принадлежит $W^{s}(\Omega)-\Omega$, то либо $z \in(x, y)_{\varnothing}^{s}$, где $x \in W^{u}(p) u$ $y \in W^{u}(q)$ для некоторых ассочиированных граничных точек $p, q \in \Omega$ (возможно, $p=q)$, либо $z \in W_{\varnothing}^{s}(p)$ для некоторой периодической граничной точки $p \in \Omega$. 
ДокаЗАТЕЛЬСТВо. Согласно [45] $z \in W^{s}\left(x_{0}\right)$ для некоторой точки $x_{0} \in \Omega$. Так как $\Omega$ замкнуто, то либо $z \in(x, y)^{s}$ с $x, y \in \Omega$ и $(x, y)^{s} \cap \Omega=\varnothing$, либо $z$ принадлежит компоненте множества $W^{s}(p)-p, p \in \Omega$, которая не пересекается с $\Omega$. В последнем случае в силу предложения 1.2 и леммы $1.1 p$ - граничная периодическая точка и $z \in W_{\varnothing}^{s}(p)$. Если $z \in(x, y)^{s}$, то в силу предложения 1.2 и леммы 1.1 $x \in W^{u}(p)$ и $y \in W^{u}(q)$ для некоторых ассоциированных граничных точек $p, q \in \Omega$.

ПРЕДЛОЖЕНИЕ 1.5. Пусть $\Omega$ - растязивающийся аттрактор (ориентируемый или неориентируемый) $A$-диффеоморфизма $f: M^{n} \rightarrow M^{n}, n \geqslant 2$. Тогда для любой точки $x \in \Omega$ достижимые из $W^{s}(x)-\left(\Omega \cap W^{s}(x)\right)$ точки канторова множества $W^{s}(x) \cap \Omega$ принадлежат неустойчивым инвариантным многообразиям (коразмерности один) граничных периодических точек.

ДокАЗАТЕЛЬСТВо вытекает из леммы 1.1 и предложения 1.4.

1.8. Характеристические сферы и связывающие цилиндры. Пусть $\Omega$ - ориентируемьй растягивающийся аттрактор коразмерности один $A$-диффеоморфизма $f: M \rightarrow M$, и пусть $B_{p q}-2$-связка аттрактора $\Omega$, состояшая из двух неустойчивых многообразий $W^{u}(p)$ и $W^{u}(q)$ ассоциированных граничных периодических точек $p$ и $q$ соответственно. В силу леммы 1.1 для каждой точки $x \in$ $W^{u}(p)-p$ имеется единственная точка $y \in W^{u}(q)-q$ такая, что $(x, y)^{s}=(x, y)_{\varnothing}^{s}$, и, обратно, для каждой точки $x \in W^{u}(q)-q$ имеется единственная точка $y \in$ $W^{u}(p)-p$ c $(x, y)^{s}=(x, y)_{\varnothing}^{s}$. Определим отображение

$$
\varphi_{p q} \stackrel{\text { def }}{=} \varphi:\left(W^{u}(p)-p\right) \cup\left(W^{u}(q)-q\right) \rightarrow\left(W^{u}(p)-p\right) \cup\left(W^{u}(q)-q\right),
$$

положив $\varphi(x)=y$, где $(x, y)^{s}=(x, y)_{\varnothing}^{s}$. Тогда

$$
\varphi\left(W^{u}(p)-p\right)=W^{u}(q)-q \text { и } \varphi\left(W^{u}(q)-q\right)=W^{u}(p)-p,
$$

т.е. отображение $\varphi$ переводит проколотые неустойчивые многообразия 2-связки друг в друга и является инволюцией. В силу теоремы о непрерывной зависимости инвариантных многообразий на компактных множествах отображение $\varphi$ является гомеоморфизмом. Из инвариантности семейства устойчивых многообразий точек базисного множества $\Omega$ вытекает соотношение

$$
\left.f^{m n} \circ \varphi\right|_{\left(W^{u}(p)-p\right) \cup\left(W^{u}(q)-q\right)}=\left.\varphi \circ f^{m n}\right|_{\left(W^{u}(p)-p\right) \cup\left(W^{u}(q)-q\right)},
$$

где $n \in \mathbb{Z}$ и $m=m(p, q)$ - период точек $p, q$.

Стандартная $k$-мерная сфера $\Sigma^{k}$ задается равенствами

$$
x_{1}^{2}+\cdots+x_{k+1}^{2}=1, \quad x_{k+2}=\cdots=0 ;
$$

$k$-мерной сферой называется множество, гомеоморфиное $\Sigma^{k} ; k$-мерная сфера $S^{k} \subset$ $M^{k}$ называется цилиндрически вложенной в $(k+1)$-мерное многообразие $M^{k}$, если существует гомеоморфное отображение $h$ произведения $\Sigma^{k} \times[-1,+1]$ в $M^{k}$ такое, что $h\left(\Sigma^{k} \times 0\right)=S^{k}$.

Так как ограничение $\left.f^{m}\right|_{W^{u}(p)}$ имеет ровно одну гиперболическую отталкивающую неподвижную точку $p$, то сушествует замкнутый $(n-1)$-мерньй шар $D_{p} \subset W^{u}(p)$, ограниченный гладко вложенной в $W^{u}(p)(n-2)$-мерной сфферой $S_{p}^{n-2}=\partial D_{p}$, такой, что $p \in \operatorname{int}\left(D_{p}\right)=D_{p}-\partial D_{p}$ и $D_{p} \subset \operatorname{int}\left(f^{m}\left(D_{p}\right)\right)$. 
Тогда множество $C_{p q}=\bigcup_{x \in \partial D_{p}}(x, \varphi(x))_{\varnothing}^{s}$ гомеоморфно открытому $(n-1)$-мерному цилиндру $\Sigma^{n-2} \times(0,1)$. Действительно, пусть $\nu: S_{p}^{n-2} \rightarrow \Sigma^{n-2}-$ произвольный гомеоморфизм. Обозначим через $\lambda_{x}(z)$ длину отрезка $(x, z)^{s} \subset(x, y)_{\varnothing}^{s}$. В силу непрерывной зависимости инвариантных многообразий от начальных условий на компактных множествах функция $\lambda_{x}(z)$ непрерывно зависит от переменных $x$ и $z$. Из компактности $S_{p}^{n-2}$ и определения связки следует, что $\lambda_{x}(\varphi(x)) \geqslant \alpha>0$ при всех $x \in S_{p}^{n-2}$. Определим отображение $\nu_{p q}: C_{p q} \rightarrow \Sigma^{n-2} \times(0,1)$, положив

$$
\nu_{p q}(z)=\left(\nu(x), \frac{\lambda_{x}(z)}{\lambda_{x}(\varphi(x))}\right), \quad z \in(x, \varphi(x))_{\varnothing}^{s} .
$$

Так как устойчивые многообразия попарно не пересекаются, то $\nu_{p q}$ является взаимно однозначным отображением. Из непрерывности $\lambda_{x}(z)$ и того, что $(n-2)$-мерные сферы $S_{p}^{n-2}$ и $S_{q}^{n-2}=\varphi\left(S_{p}^{n-2}\right)$ находятся друг от друга на ненулевом расстоянии, вытекает, что $\nu_{p q}$ - гомеоморфизм. Множество $C_{p q}$ мы будем называть связывающим иилиндром.

Так как $\varphi$ - гомеоморфизм, то $S_{q}^{n-2}=\varphi\left(S_{p}^{n-2}\right)$ является цилиндрически вложенной в $W^{u}(q)(n-2)$-мерной сферой. Согласно обобшенной теореме Шенфлиса $\left[18\right.$, гл. 5] $S_{q}^{n-2}$ ограничивает в $W^{u}(q)(n-1)$-мерный шар, который мы обозначим через $D_{q}$. Из равенства (1.1) вытекает, что $q \in \operatorname{int}\left(D_{q}\right)$. Множество $S_{p q}=D_{p} \cup D_{q} \cup C_{p q}$ гомеоморфно $(n-1)$-мерной сфере, которую мы будем называть характеристической сферой, соответствующей связке $B_{p q}=W^{u}(p) \cup$ $W^{u}(q)$. В силу теоремы о структуре произведения и непрерьвной зависимости инвариантных многообразий от начальных условий, $S_{p q}$ является цилиндрически вложенной в $M$ сфиерой. Отметим, что характеристическая сфеера не определяется однозначно связкой.

1.9. Группа голономии. Для полноты изложения приведем здесь определение тривиальности группы голономии. Будем следовать работе [20], где имеется наиболее геометрическое, на наш взгляд, определение.

Пусть $L \in \mathscr{L}$ - слой ламинации $\mathscr{L}$. Для простоты будем рассматривать случай ламинации $\mathscr{L}$ коразмерности один. Возьмем произвольную точку $x_{0} \in L$ и петлю $\gamma \subset L$ с начальной точкой $x_{0}$, представляющей элемент $[\gamma]$ фундаментальной группы $\pi_{1}\left(L, x_{0}\right)$. Не уменьшая обшности, можно считать, что $\gamma$ является гладкой кривой. Восстановим в каждой точке $x \in \gamma$ нормаль $l_{x}$ к слою $L$. Это семейство нормалей можно построить таким образом, чтобы оно образовывало кольцо $A=\bigcup_{x \in \gamma} l_{x}$, трансверсальное слоям ламинации $\mathscr{L}$. Поэтому $A$ пересекается со слоями из $\mathscr{L}$ вдоль кривых (в частности, вдоль кривой $\gamma$ ). Если все достаточно близкие к $\gamma$ кривые замкнуты, то будем говорить, что $\gamma$ является непредельныцм циклом. Из непрерывной зависимости слоев от начальных условий на компактных множествах вытекает, что свойство кривой $\gamma$ быть непредельным циклом не зависит от выбора представителя элемента $[\gamma]$ и не меняется при свободной гомотопии $\gamma$, при которой начальная точка $x_{0}$ движется по любому пути на $L$ (см. $[20$, лемма 2.1]).

Таким образом, свойство замкнутой кривой на $L$ быть непредельным циклом есть инвариант свободной гомотопии, что влечет корректность следующего определения. Элемент $[\gamma] \in \pi_{1}(L)$ является непредельным ииклом, если любой его представитель есть непредельный цикл. Будем говорить, что $L \in \mathscr{L}$ имеет тривиальную группу голономии, если все элементы его фундаментальной группы $\pi_{1}(L)$ являются непредельными циклами. 


\section{§2. Характеристические сферы и связывающие цилиндры}

Везде в этом параграфе $\Omega$ - ориентируемьй растягивающийся аттрактор коразмерности один $A$-диффеоморфизма $f: M \rightarrow M n$-мерного замкнутого многообразия $M=M^{n}, n \geqslant 3$. Как уже отмечалось ранее, достижимая из $M-\Omega$ граница аттрактора $\Omega$ состоит из конечного числа неустойчивых $(n-1)$-мерных многообразий граничных периодических точек. Для каждой пары ассоциированных граничных периодических точек построим из дуг одномерных устойчивых многообразий связывающий $(n-1)$-мерный цилиндр и соответствующую $(n-1)$-мерную сфферу. Как будет показано в следующем параграфе, свойство этих сфер ограничивать в многообразии $n$-мерный шар сушественно влияет на гомотопический и топологический тип многообразия.

2.1. Построение характеристических сфер. Пусть $B_{p q}-2$-связка ориентируемого растягивающегося аттрактора $\Omega$, состоящая из двух неустойчивых многообразий $W^{u}(p)$ и $W^{u}(q)$ ассоциированных граничных периодических точек $p$ и $q$ соответственно, и $m=m(p, q)$ - период точек $p, q$. Так как ограничение $\left.f^{m}\right|_{W^{u}(p)}$ имеет ровно одну гиперболическую отталкивающую неподвижную точку $p$, то сушествует замкнутый $(n-1)$-мерный шар $D_{p} \subset W^{u}(p)$, ограниченный гладко вложенной в $W^{u}(p)(n-2)$-мерной сферой $S_{p}^{n-2}=\partial D_{p}$, такой, что

i) $p \in \operatorname{int}\left(D_{p}\right)=D_{p}-\partial D_{p}, D_{p} \subset \operatorname{int}\left(f^{m}\left(D_{p}\right)\right)$.

В частности, $S_{p}^{n-2} \cap f^{m}\left(S_{p}^{n-2}\right)=\varnothing$ и, следовательно, $S_{p}^{n-2}$ лежит внутри сферы $f^{m}\left(S_{p}^{n-2}\right)$. Так как обе сферы $S_{p}^{n-2}$ и $f^{m}\left(S_{p}^{n-2}\right) C^{1}$-вложены, то они удовлетворяют еще одному условию:

ii) $(n-2)$-мерные сферы $S_{p}^{n-2}$ и $f^{m}\left(S_{p}^{n-2}\right)$ ограничивают в неустойчивом многообразии $W^{u}(p)(n-1)$-мерное кольцо $A_{p}$, гомеоморфное $\Sigma^{n-2} \times[0,1]$ (см. рис. 4$)$.

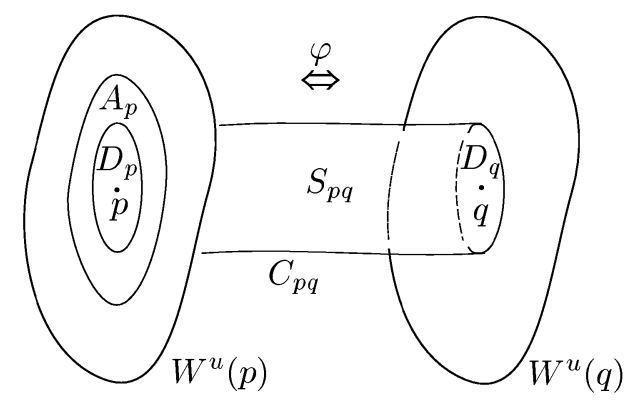

Рис. 4. Характеристическая сфера $S_{p q}=C_{p q} \cup D_{p} \cup D_{q}$

ЛЕмма 2.1. Отображсене $\varphi_{p q}$ продолжается до гомеоморфизма

$$
\varphi_{p q}: W^{u}(p) \cup W^{u}(q) \rightarrow W^{u}(p) \cup W^{u}(q),
$$

если положить $\varphi_{p q}(p)=q u \varphi_{p q}(q)=p$.

ДокАЗАТЕльство. Так как $\left.\varphi_{p q}\right|_{W^{u}}(p)-p$ - гомеоморфизм, то $\varphi_{p q}\left(S_{p}^{n-2}\right)-$ цилиндрически вложенная в $W^{u}(q)(n-2)$-сфера, которую мы обозначим через $S_{q}^{n-2}$. Из обобщенной теоремы Шенфлиса [36] (см. также [18, гл. 5]) следует, что $S_{q}^{n-2}$ ограничивает в $W^{u}(q)$ замкнутьй $(n-1)$-шар $D_{q} \subset W^{u}(q)$. 
Так как $q$ - единственная отталкиваюшая точка ограничения $\left.f^{m}\right|_{W^{u}(q)-q}$, то сушествует $j_{0} \in \mathbb{N}$ такое, что $D_{q} \subset f^{j_{0} m}\left(D_{q}\right)$. Из (1.1) получаем

$$
\varphi_{p q} \circ f^{m j_{0}}\left(S_{p}^{n-2}\right)=f^{m j_{0}} \circ \varphi_{p q}\left(S_{p}^{n-2}\right)=f^{j_{0} m}\left(S_{q}^{n-2}\right) \subset W^{u}(q)-D_{q} .
$$

Заметим, что

$$
D_{p} \subset \operatorname{int}\left(f^{m}\left(D_{p}\right)\right) \subset \operatorname{int}\left(f^{m j_{0}}\left(D_{p}\right)\right), \quad f^{m j_{0}}\left(S_{p}^{n-2}\right) \subset W^{u}(p)-D_{p} .
$$

Следовательно, $\varphi_{p q}\left(D_{p}-p\right)=D_{q}-q$. Отсюда получаем, что

$$
\varphi_{p q} \circ f^{j m}\left(D_{p}-p\right)=f^{j m} \circ \varphi_{p q}\left(D_{p}-p\right)=f^{j m}\left(D_{q}-q\right) .
$$

Аналогично, покажем, что

$$
\varphi_{p q} \circ f^{j m}\left(D_{q}-q\right)=f^{j m} \circ \varphi_{p q}\left(D_{q}-q\right)=f^{j m}\left(D_{p}-p\right) .
$$

Отсюда вытекает требуемьй результат, поскольку

$$
\bigcap_{j \leqslant 0} f^{j m}\left(D_{p}\right)=p, \quad \bigcap_{j \leqslant 0} f^{j m}\left(D_{q}\right)=q .
$$

СЛЕДСТВИЕ 2.1. Выполняются следующие условия:

1) $q \in \operatorname{int}\left(D_{q}\right), \quad D_{q} \subset \operatorname{int}\left(f^{m}\left(D_{q}\right)\right)$;

2) $(n-2)$-сферь $S_{q}^{n-2}=\varphi_{p q}\left(S_{p}^{n-2}\right)$ и $f^{m}\left(S_{q}^{n-2}\right)$ ограничивают замкнутое $(n-1)$-кольцо $A_{q}=\varphi_{p q}\left(A_{p}\right)$, гомеоморфное $\Sigma^{n-2} \times[0,1]$.

Более того, выполняется следующее соотношение:

$$
\left.f^{m j} \circ \varphi_{p q}\right|_{W^{u}(p) \cup W^{u}(q)}=\left.\varphi_{p q} \circ f^{m j}\right|_{W^{u}(p) \cup W^{u}(q)}
$$

для любого $j \in \mathbb{Z}$.

Предположим, что точки $x, z$ лежат на одном устойчивом многообразии некоторой точки из $\Omega$. Обозначим через $\lambda(x, z)$ длину дуги $(x, z)^{s}$. Следующую лемму получим из наличия локальной структуры произведения и теоремы о непрерывной зависимости инвариантных многообразий от начальных условий.

Лемма 2.2. Функиия $\lambda(x, z)$ непрерывно зависит от переменных $x, z$.

ДоказАтельство. Существует $j_{0} \in \mathbb{N}$ такое, что дуга $f^{j_{0}}(x, z)^{s}=\left(f^{j_{0}}(x)\right.$, $\left.f^{j_{0}}(y)\right)^{s}$ лежит в окрестности множества $\Omega$ с локальной структурой произведения [45]. Следовательно, имеется трубчатая окрестность $U\left((x, z)^{s}\right)$ дуги $(x, z)^{s}$, гомеоморфная прямому произведению $\mathbb{B}^{n-1}$ и $(0,1)$, причем дуги устойчивых многообразий соответствуют при этом гомеоморфизме отрезкам $b \times(0,1), b \in \mathbb{B}^{n-1}$ [44]. Отсюда следует требуемое утверждение.

ЛЕмма 2.3. Множество $C_{p q}=\bigcup_{x \in S_{p}^{n-2}}\left(x, \varphi_{p q}(x)\right)_{\varnothing}^{s}$ гомеоморфно откритому $(n-1)$-мерному иилиндру $\Sigma^{n-2} \times(0,1)$. 
ДокАЗАТЕЛЬСТво. Пусть $\nu: S_{p}^{n-2} \rightarrow \Sigma^{n-2}-$ произвольньй гомеоморфизм. Согласно лемме 2.2 длина $\lambda(x, z)$ дуг $(x, z)^{s} \subset(x, y)_{\varnothing}^{s}, x \in S_{p}^{n-2}$, непрерывно зависит от $x$ и $z$. Более того, $\lambda_{x}\left(\varphi_{p q}(x)\right) \geqslant \alpha$ для всех $x \in S_{p}^{n-2}$ и некоторого $\alpha>0$, поскольку $S_{p}^{n-2}$ компактно.

Определим отображение $\nu_{p q}: C_{p q} \rightarrow \Sigma^{n-2} \times(0,1)$, положив

$$
\nu_{p q}(z)=\left(\nu(x), \frac{\lambda(x, z)}{\lambda\left(x, \varphi_{p q}(x)\right)}\right), \quad z \in\left(x, \varphi_{p q}(x)\right)_{\varnothing}^{s} .
$$

Это отображение взаимно однозначное, так как инвариантные многообразия попарно не пересекаются. Поскольку $\lambda(x, z)$ непрерьвна и $S_{p}^{n-2} \cap S_{q}^{n-2}=\varnothing$, то $\nu_{p q}$ - гомеоморфизм.

СлЕДСТвиЕ 2.2. Множество

$$
\operatorname{clos} C_{p q}=C_{p q} \cup S_{p}^{n-2} \cup S_{q}^{n-2}=\bigcup_{x \in S_{p}^{n-2}}\left[x, \varphi_{p q}(x)\right]_{\varnothing}^{s}
$$

гомеоморфно замкнутому $(n-1)$-мерному иилиндру $\Sigma^{n-2} \times[0,1]$.

Цилиндр $C_{p q}=\bigcup_{x \in \partial D_{p}}\left(x, \varphi_{p q}(x)\right)_{\varnothing}^{s}$ называется связывающим иилиндром, соответствующим связке $B_{p q}=W^{u}(p) \cup W^{u}(q)$.

Лемма 2.4. Множество $S_{p q}=D_{p} \cup D_{q} \cup C_{p q}$ гомеоморфно $(n-1)$-мерной cферe $\Sigma^{n-1}$.

ДокаЗАТЕЛЬСТво. В силу построения $D_{p} \cap D_{q}=\varnothing, D_{p} \cap C_{p q}=\varnothing$ и $D_{q} \cap$ $C_{p q}=\varnothing$. Отсюда и из следствия 2.2 получаем требуемьй результат.

Сфера $S_{p q}=D_{p} \cup D_{q} \cup C_{p q}$ называется характеристической сферой, соответствующей связке $B_{p q}=W^{u}(p) \cup W^{u}(q)$. Отметим, что характеристическая сффера и связываюший цилиндр выбираются неоднозначно для связки $B_{p q}$. Они зависят от выбора $(n-1)$-шара $D_{p}$ (см. рис. 4$)$. В силу наличия структуры произведения и теоремы о непрерьвной зависимости инвариантных многообразий от начальных условий, $S_{p q}$ является цилиндрически вложенной в $M$ сферой.

2.2. Свойства характеристических сфер. Будем придерживаться обозначений предыдушего пункта. Напомним, что $(n-2)$-сферы $S_{p}^{n-2}$ и $f^{m}\left(S_{p}^{n-2}\right)$ ограничивают в $W^{u}(p)$ замкнутое $(n-1)$-мерное кольцо $A_{p}$, гомеоморфное $\Sigma^{n-2} \times[0,1]$. В силу равенств

$$
W^{u}(p)-p=\bigcup_{j \in \mathbb{Z}} f^{j m}\left(A_{p}\right), \quad \operatorname{int}\left(f^{j m}\left(A_{p}\right)\right) \cap \operatorname{int}\left(f^{k m}\left(A_{p}\right)\right)=\varnothing, \quad j \neq k,
$$

$A_{p}$ называется фундаментальным кольцом преобразования $\left.f^{m}\right|_{W^{u}(p)}$. Из следствия 2.1 вытекает, что $A_{q}=\varphi\left(A_{p}\right)$ есть фундаментальное кольцо преобразования $\left.f^{m}\right|_{W^{u}(q)}$, т.e.

$$
W^{u}(q)-q=\bigcup_{j \in \mathbb{Z}} f^{j m}\left(A_{q}\right), \quad \operatorname{int}\left(f^{l m}\left(A_{q}\right)\right) \cap \operatorname{int}\left(f^{k m}\left(A_{q}\right)\right)=\varnothing, \quad l \neq k .
$$

Положим

$$
A_{p q} \stackrel{\text { def }}{=} \bigcup_{x \in A_{p}}[x, y]_{\varnothing}^{s}=\bigcup_{x \in A_{p}}\left[x, \varphi_{p q}(x)\right]_{\varnothing}^{s}=\bigcup_{x \in A_{q}}\left[x, \varphi_{p q}(x)\right]_{\varnothing}^{s} .
$$


ЛЕмма 2.5. Существует гомеоморфизм $h_{p q}: A_{p q} \rightarrow A_{p} \times[0,1]$ такой, что

$$
h_{p q}\left([x, y]_{\varnothing}^{s}\right)=\{x\} \times[0,1] \quad \text { для любого } \quad x \in A_{p} .
$$

ДокАЗАТЕЛЬСТво. Пусть $\lambda(x, z)$ - длина дуги $[x, z]^{s} \subset(x, y)_{\varnothing}^{s}$. Определим $h_{p q}$ в виде

$$
h_{p q}(z)=\left(x, \frac{\lambda(x, z)}{\lambda\left(x, \varphi_{p q}(x)\right)}\right), \quad z \in\left[x, \varphi_{p q}(x)\right]^{s} \subset A_{p q} .
$$

Так как дуги $\left[x, \varphi_{p q}(x)\right]_{\varnothing}^{s},\left[x^{\prime}, \varphi_{p q}\left(x^{\prime}\right)\right]_{\varnothing}^{s}, x \neq x^{\prime}$, попарно не пересекаются, то $h_{p q}$ взаимно однозначное. Потому в силу леммы $2.2 h_{p q}$-гомеоморфизм.

Как следствие, $A_{p q}$ гомеоморфно $n$-мерному кольцу $\Sigma^{n-2} \times[0,1]^{2}$. Положим

$$
D_{p q}\left(S_{p q}\right) \stackrel{\text { def }}{=} D_{p q}=\bigcup_{j \geqslant 0} f^{j m}\left(A_{p q}\right) .
$$

Из (2.2) и (2.3) следует

$$
f^{j m}\left(C_{p q}\right) \subset D_{p q}, \quad j \geqslant 0 .
$$

ЛЕмма 2.6. Имеет место равенство $D_{p q}=\bigcup_{x \in W^{u}(p)-\operatorname{int} D_{p}}\left[x, \varphi_{p q}(x)\right]_{\varnothing}^{s}$.

ДокАЗАТЕЛЬСТво. Из равенств

$$
A_{p q}=\bigcup_{x \in A_{p}}[x, y]_{\varnothing}^{s}, \quad W^{u}(p)-\operatorname{int} D_{p}=\bigcup_{j \geqslant 0} f^{j m}\left(A_{p}\right)
$$

получаем

$$
D_{p q} \subset \bigcup_{x \in W^{u}(p)-\operatorname{int} D_{p}}\left[x, \varphi_{p q}(x)\right]_{\varnothing}^{s} .
$$

Возьмем $\left[x^{\prime}, \varphi_{p q}\left(x^{\prime}\right)\right]_{\varnothing}^{s} \mathrm{c} x^{\prime} \in W^{u}(p)-\operatorname{int} D_{p}$. Из (2.2) вытекает, что $x^{\prime}=f^{j m}(x)$ для некоторого $j \geqslant 0$ и $x \in W^{u}(p)-\operatorname{int} D_{p}$. Поэтому

$$
\bigcup_{x \in W^{u}(p)-\operatorname{int} D_{p}}\left[x, \varphi_{p q}(x)\right]_{\varnothing}^{s} \subset D_{p q} .
$$

Пусть $\pi_{p}: D_{p q} \rightarrow W^{u}(p)-\operatorname{int} D_{p}-$ проекция, переводящая точку $a \in D_{p q}$ в $x$, если $a \in[x, y]_{\varnothing}^{s} \subset D_{p q}$. Согласно лемме 2.6 проекция $\pi_{p}$ определена корректно, поскольку дуги $\left[x, \varphi_{p q}(x)\right]_{\varnothing}^{s},\left[x^{\prime}, \varphi_{p q}\left(x^{\prime}\right)\right]_{\varnothing}^{s}$ попарно не пересекаются при $x \neq x^{\prime}$.

По построению множества $f^{j_{1} m}\left(A_{p q}\right), f^{j_{2} m}\left(A_{p q}\right)$ имеют попарно непересекаюшиеся внутренности при различных $j_{1}, j_{2}$ и пересекаются по множеству, гомеоморфному $\Sigma^{n-2} \times[0,1]$, только в том случае, если $\left|j_{1}-j_{2}\right|=1$. Поэтому на множестве $D_{p q}=\bigcup_{j \geqslant 0} f^{j m}\left(A_{p q}\right)$ корректно вводится топология, индуцированная стандартной топологией на $A_{p q}$ как на гомеоморфном образе множества $\Sigma^{n-2} \times[0,1]^{2}$.

Лемма 2.7. Тройка $\left(D_{p q}, W^{u}(p)-\operatorname{int} D_{p}, \pi_{p}\right)$ является тривиальныцм расслоением со слоем $[0 ; 1]$. 
ДокАЗАТЕЛЬСтво. Заметим, что из леммы 2.5 вытекает, что тройка $\left(D_{p q}\right.$, $\left.W^{u}(p)-\operatorname{int} D_{p}, \pi_{p}\right)$ является расслоением со слоем $[0 ; 1]$, поскольку $D_{p q}=$ $\bigcup_{j \geqslant 0} f^{j m}\left(A_{p q}\right)$ и $A_{p q} \cong A_{p} \times[0 ; 1]$ есть расслоение со слоем [0;1]. Чтобы доказать тривиальность расслоения, построим послойное отображение

$$
H_{p q}: D_{p q} \rightarrow\left(W^{u}(p)-\operatorname{int} D_{p}\right) \times[0,1]
$$

которое осуществляет изоморфизм с расслоением-произведением

$$
\left(\left(W^{u}(p)-\operatorname{int} D_{p}\right) \times[0,1], W^{u}(p)-\operatorname{int} D_{p}, \operatorname{pr}_{1}\right),
$$

где $\operatorname{pr}_{1}:\left(W^{u}(p)-\operatorname{int} D_{p}\right) \times[0,1] \rightarrow W^{u}(p)-\operatorname{int} D_{p}-$ проекция на первый множитель. Пусть

$$
H_{p q}(z)=\left(x, \frac{\lambda(x, z)}{\lambda\left(x, \varphi_{p q}(x)\right)}\right), \quad z \in\left[x, \varphi_{p q}(x)\right]^{s} \subset D_{p q} .
$$

Отсюда непосредственно вытекает, что $H_{p q}$ отображает $\pi_{p}^{-1}(x)$ в $\{x\} \times[0,1]$, где $x \in W^{u}(p)-\operatorname{int} D_{p}$. Таким образом, $H_{p q}$ является послойным отображением. Так как дуги $\left[x, \varphi_{p q}(x)\right]_{\varnothing}^{s},\left[x^{\prime}, \varphi_{p q}\left(x^{\prime}\right)\right]_{\varnothing}^{s}$ при $x \neq x^{\prime}$ попарно не пересекаются, то $H_{p q}$ взаимно однозначное. В силу леммы $2.2 H_{p q}$-гомеоморфизм.

СлеДСТВИЕ 2.3. Вложения $S_{p}=\partial D_{p} \subset D_{p q}, S_{q}=\partial D_{q} \subset D_{p q}$ индуиируют изоморфизмы групп

$$
\pi_{n-2}\left(S_{p}\right) \rightarrow \pi_{n-2}\left(D_{p q}\right), \quad \pi_{n-2}\left(S_{q}\right) \rightarrow \pi_{n-2}\left(D_{p q}\right) \cong \mathbb{Z}
$$

соответственно. Более того, обе сферы $S_{p}, S_{q}$ гомотопически әквивалентнbl $D_{p q}$.

ДокаЗАТЕЛЬСТво. Согласно лемме $2.7 W^{u}(p)-\operatorname{int} D_{p}$ есть ретракт множества $D_{p q}$. Осталось заметить, что $S_{p}$ является ретрактом $W^{u}(p)-\operatorname{int} D_{p}$, поскольку $W^{u}(p) \cong \mathbb{R}^{n-1}$.

Возьмем теперь две сферы $S_{p q}=D_{p} \cup D_{q} \cup C_{p q}$ и $S_{p q}^{\prime}=D_{p}^{\prime} \cup D_{q}^{\prime} \cup C_{p q}^{\prime}$, соответствуюшие одной и той же 2-связке $B_{p q}$.

Лемма 2.8. Характеристические сферы $S_{p q}, S_{p q}^{\prime}$ изотопны в $M^{n}$. В частности, $S_{p q}$ ограничивает шар в $M^{n}$, если и только если $S_{p q}^{\prime}$ ограничивает шар в $M^{n}$.

ДокАЗАТЕльСтво. Напомним, что сферы $S_{p}=\partial D_{p}$ и $S_{p}^{\prime}=\partial D_{p}^{\prime} C^{1}$-вложены в $W^{u}(p)$ и, в частности, они цилиндрически вложены. Поэтому сушествует изотопия $F_{t}: W^{u}(p) \rightarrow W^{u}(p), 0 \leqslant t \leqslant 1$, переводящая $S_{p}$ в $S_{p}^{\prime}$ [53] (см. также [18]). Более того, можно построить $F_{t}$ таким образом, чтобы $F_{t}(p)=p$ для всех $t$, поскольку $p$ лежит внутри обеих сфер $S_{p}, S_{p}^{\prime}$. Следовательно, $F_{t}$ продолжается до изотопии цилиндров $C_{p q}, C_{p q}^{\prime}$ и сфер $S_{q}=\partial D_{q}, S_{q}^{\prime}=\partial D_{q}^{\prime}$. Отсюда получаем, что $S_{p q}$ изотопна $S_{p q}^{\prime}$.

Напомним, что граничные периодические точки ориентируемого растягивающегося аттрактора $\Omega$ коразмерности один распадаются на пары ассоциированных 
точек. Это распадение соответствует распадению граничных неустойчивых многообразий базисного множества $\Omega$ на 2 -связки. Пусть $\left\{p_{i}, q_{i}\right\}_{i=1}^{k}-$ пары ассоциированных граничных точек $\Omega$. Каждой паре $p_{i}, q_{i}$ однозначно соответствует 2-связка $B_{p_{i} q_{i}}=W^{u}\left(p_{i}\right) \cup W^{u}\left(q_{i}\right)$. Обозначим через $S_{p_{i} q_{i}}$ характеристическую сферу, соответствуюшую $B_{p_{i}} q_{i}$ и состоящую из связываюшего $(n-1)$-мерного цилиндра $C_{p_{i} q_{i}}$ и замкнутых $(n-1)$-шаров $D_{p_{i}} \subset W^{u}\left(p_{i}\right), D_{q_{i}} \subset W^{u}\left(q_{i}\right)$, где $D_{p_{i}} \subset \operatorname{int} f^{m_{i}}\left(D_{p_{i}}\right), D_{q_{i}} \subset \operatorname{int} f^{m_{i}}\left(D_{q_{i}}\right)$, и $m_{i}=m\left(p_{i}, q_{i}\right)$ - обший период точек $p_{i}, q_{i}, i=1, \ldots, k$. Сферы $S_{p_{i}}^{n-2}=\partial D_{p_{i}}, f^{m_{i}}\left(S_{p_{i}}^{n-2}\right)$ ограничивают в $W^{u}\left(p_{i}\right)$ замкнутое $(n-1)$-мерное кольцо $A_{p_{i}}$, гомеоморфное $\Sigma^{n-2} \times[0,1]$. Имеем

$$
A_{p_{i} q_{i}}=\bigcup_{x \in A_{p_{i}}}\left[x, \varphi_{p_{i} q_{i}}(x)\right]_{\varnothing}^{s} \cong A_{p_{i}} \times[0,1] \cong \Sigma^{n-2} \times[0,1]^{2}
$$

где гомеоморфизм

$$
\varphi_{p_{i} q_{i}}=\varphi_{i}: W^{u}\left(p_{i}\right) \cup W^{u}\left(q_{i}\right) \rightarrow W^{u}\left(p_{i}\right) \cup W^{u}\left(q_{i}\right)
$$

удовлетворяет равенству (2.1). В силу леммы 2.6

$$
D_{p_{i} q_{i}} \stackrel{\text { def }}{=} \bigcup_{j \geqslant 0} f^{j m}\left(A_{p_{i} q_{i}}\right)=\bigcup_{x \in W^{u}\left(p_{i}\right)-\operatorname{int} D_{p_{i}}}\left[x, \varphi_{i}(x)\right]_{\varnothing}^{s} .
$$

ТЕОРемА 2.1. Пусть $\Omega$ - ориентируемый растягивающийся аттрактор коразмерности один, и пусть $\left\{p_{i}, q_{i}\right\}_{i=1}^{k}-$ пары ассоциированных граничных точек множества $\Omega$. Тогда имеют место следующие свойства:

1) множество

$$
D(\Omega) \stackrel{\text { def }}{=} \bigcup_{i=1}^{k} \bigcup_{j \geqslant 0} f^{j m_{i}}\left(A_{p_{i} q_{i}}\right) \cup \Omega=\bigcup_{i=1}^{k} D_{p_{i} q_{i}} \cup \Omega
$$

является топологическим п-мерным подмногообразием с границей $\partial D(\Omega)=$ $\bigcup_{i=1}^{k} S_{p_{i} q_{i}} ;$ в частности, семейство характеристических сфер $\bigcup_{i=1}^{k} S_{p_{i} q_{i}}$ разбивает $M=M^{n}$, более того, любая компонента подмногообразия $D(\Omega)$ содержит ровно одну $C$-плотную компоненту множества $\Omega$ и, обратно, любая $C$-плотная компонента множества $\Omega$ принадлежит ровно одной компоненте $D(\Omega)$;

2) если каждая характеристическая сфера $S_{p_{i} q_{i}}$ ограничивает шар $E_{p_{i} q_{i}} \subset$ $M, m o$

$$
M=\bigcup_{i=1}^{k} E_{p_{i} q_{i}} \cup W^{s}(\Omega)=\bigcup_{i=1}^{k} \bigcup_{j \geqslant 0} f^{j m_{i}}\left(E_{p_{i} q_{i}}\right) \cup \Omega,
$$

более того, $\Omega$ является $C$-плотным базисным множеством. 
ДокаЗАТЕЛЬСтво. Не уменьшая общности, можно считать, что все периодические граничные точки $\left\{p_{i}, q_{i}\right\}_{i=1}^{k}$ являются неподвижными (чтобы этого достичь, достаточно перейти к некоторой степени диффеоморфизма $f$ ). Сперва покажем, что первое утверждение теоремы следует из второго. Разрезав $M$ вдоль характеристических сфер, получим конечное число топологических $n$-многообразий $M_{1}, \ldots M_{l}$.

Предположим, что $M_{1}$ содержит граничные неустойчивые многообразия некоторых граничных неподвижных точек множества $\Omega$, и пусть $\Omega_{1} \subset M_{1} \cap \Omega$ - часть $\Omega$, принадлежашая $M_{1}$ и состоящая из целых неустойчивых многообразий, т.е. если $W^{u} \cap \Omega_{1} \neq \varnothing$, то $W^{u} \subset M_{1}$. Пусть $p$ - граничная неподвижная точка с $W^{u}(p) \subset M_{1}$. В силу построения характеристической сферы $S_{p q}$ имеем, что $\left(W^{u}(q)-D_{q}\right) \cap M_{1} \neq \varnothing$, где $q$ - граничная неподвижная точка, ассоциированная с $p$. Множество $W^{u}(q)-D_{q}$ не пересекает связываюших цилиндров базисного множества $\Omega$. Поэтому $W^{u}(q)-D_{q}$ не пересекает границу $\partial M_{1}$. Следовательно, $W^{u}(q) \subset M_{1}$. Таким образом, $\Omega_{1}$ содержит граничное неустойчивое многообразие $W^{u}(p)$ тогда и только тогда, когда $\Omega_{1}$ содержит граничное неустойчивое многообразие $W^{u}(q)$, где $p, q$ - ассоциированные точки. Перенумеровав ассоциированные точки множества $\Omega$, можно считать, что ассоциированные точки $p_{1}, q_{1}, \ldots, p_{k_{1}}, q_{k_{1}}$ и их неустойчивые многообразия $W^{u}\left(p_{1}\right), W^{u}\left(q_{1}\right), \ldots, W^{u}\left(p_{k_{1}}\right), W^{u}\left(q_{k_{1}}\right)$ лежат в $M_{1}$. По построению $\partial M_{1}$ содержит $(n-1)$-сферы $S_{p_{1} q_{1}}, \ldots, S_{p_{k_{1}} q_{k_{1}}}$.

Возьмем неустойчивое многообразие $W^{u}(x) \subset \Omega$, которое не совпадает ни с одним из $W^{u}\left(p_{i}\right), W^{u}\left(q_{i}\right), 1 \leqslant i \leqslant k_{1}$, но пересекает $\partial M_{1}$. Так как $W^{u}(x)$ не пересекает открытых связываюших цилиндров (как и любое неустойчивое многообразие из $\Omega$ ), то из построения характеристических сфер вытекает, что пересечение $W^{u}(x) \cap M_{1}$ состоит из $(n-1)$-мерного шара $D_{r} \subset \Omega$, где $r \in \Omega$-граничная точка, отличная от $p_{i}, q_{i}, 1 \leqslant i \leqslant k_{1}$. Это рассуждение показывает, что если неустойчивое многообразие $W^{u}(y) \subset \Omega$ проходит через $y \in \operatorname{int} M_{1}$, то $W^{u}(y) \subset M_{1}$ и, более того, $W^{u}(y)$ полностью лежит в $\operatorname{int} M_{1}$. Таким образом, $\Omega_{1}$ есть объединение неустойчивых многообразий $W^{u}\left(p_{1}\right), W^{u}\left(q_{1}\right), \ldots, W^{u}\left(p_{k_{1}}\right), W^{u}\left(q_{k_{1}}\right)$ и неустойчивых многообразий из $\Omega$, которые полностью лежат в int $M_{1}$.

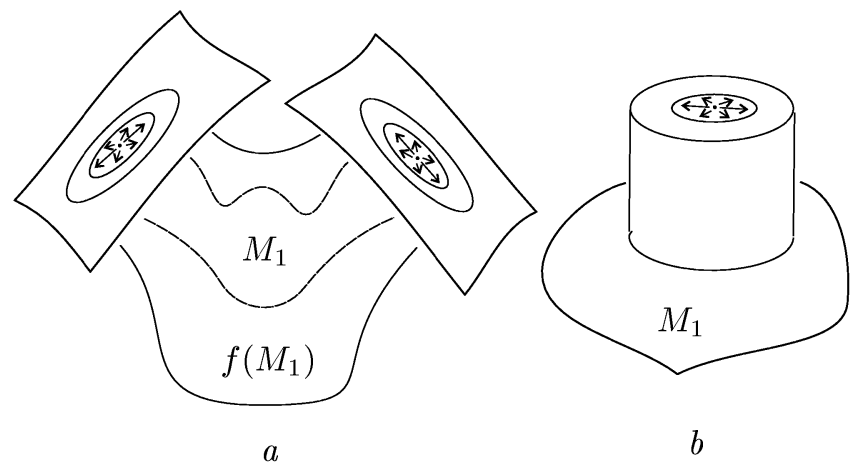

Рис. 5. $a-A_{p q} \subset f\left(M_{1}\right)-M_{1} ; b-A_{p q} \subset M_{1}-f\left(M_{1}\right)$

Теперь рассмотрим $M_{1}$ как подмножество многообразия $M^{n}$. Из соотношений (2.2)-(2.4) вытекает, что множество

$$
\widehat{M}_{1}^{+} \stackrel{\text { def }}{=} \cos \left(f\left(M_{1}\right)-M_{1}\right) \cup \operatorname{clos}\left(M_{1}-f\left(M_{1}\right)\right)
$$


есть объединение колец вида $A_{p q}$, приклеенных к гранище $\partial M_{1}$ (см. рис. 5). Следовательно, $M_{1}$ - ретракт как множества $\widehat{M}_{1}^{+} \cup M_{1}$, так и множества $M_{1}-\widehat{M}_{1}^{+}$. Возьмем произвольное неустойчивое многообразие $W^{u}(x) \subset \operatorname{int} M_{1}, x \in \Omega_{1}$. Так как $M_{1}$ линейно связное, то существует путь $\omega$, соединяющий в $M_{1}$ точки $x$ и $p_{1}$. Из локальной структуры $\Omega$ и того, что $M_{1}$ есть ретракт множества $M_{1}-\widehat{M}_{1}^{+}$, вытекает, что можно взять $\omega$ таким образом, чтобы $\omega \subset M_{1}-\widehat{M}_{1}^{+}$, поскольку $x$, $p_{1} \subset M_{1}-\widehat{M}_{1}^{+}$. Отсюда и из того, что $f^{-1}\left(p_{1}\right)=p_{1}$, следует $f^{-1}(\omega) \subset M_{1}$. Поэтому $f^{-1}(x) \in M_{1}$. Следовательно, $f^{-1}\left(W^{u}(x)\right) \subset M_{1}$. Аналогично, используя множество

$$
\widehat{M}_{1}^{-} \stackrel{\text { def }}{=} \operatorname{clos}\left(f^{-1}\left(M_{1}\right)-M_{1}\right) \cup \operatorname{clos}\left(M_{1}-f^{-1}\left(M_{1}\right)\right),
$$

покажем, что $f\left(W^{u}(x)\right) \subset M_{1}$. Так как $\Omega_{1}$ - объединение неустойчивых многообразий своих точек, то получим $f^{ \pm 1}\left(\Omega_{1}\right)=\Omega_{1}$, т.е. $\Omega_{1}$ является инвариантным множеством диффеоморфизма $f$. Аналогичное рассуждение применяется для любого базисного множества $\Lambda \subset M_{1}$, отличного от $\Omega$, и доказывается его инвариантность относительно $f, f(\Lambda)=\Lambda$, поскольку $\left(\widehat{M}_{1}^{-} \cup \widehat{M}_{1}^{+}\right) \cap \Lambda=\varnothing$.

По построению граница $\partial M_{1}$ компоненты $M_{1}$ состоит из $k_{2} \geqslant k_{1}(n-1)$-сфер. Приклеим $n$-мерные шары $B_{1}, \ldots, B_{k_{2}}$ к $M_{1}$ вдоль каждой $(n-1)$-сферы из $\partial M_{1}$. Тогда получим замкнутое $n$-многообразие, которое обозначим через $M_{1}^{\prime}$. Так как семейство колец

$$
\widehat{M}_{1}^{-} \cup \widehat{M}_{1}^{+}=\bigcup_{i=1}^{k_{1}} f\left(A_{p_{i} q_{i}}\right) \bigcup_{i=k_{1}+1}^{k_{2}} f\left(A_{p_{i} q_{i}}\right)
$$

образует трубчатую окрестность семейства цилиндров $\left\{C_{p_{i}} q_{i}\right\}_{i=1}^{k_{2}}$, то $\left.f\right|_{M_{1}}$ можно продолжить до диффеоморфизма $f_{1}: M_{1}^{\prime} \rightarrow M_{1}^{\prime}$ так, чтобы $f_{1}$ имел либо отталкивающую, либо притягивающую точку внутри каждого приклеенного $n$-шара $B_{i}$, $1 \leqslant i \leqslant k_{2}$. Отметим здесь два момента. Во-первых, приклеивание шаров не меняет (как множество) $\Omega_{1}$. Во-вторых, шары, приклеенные к $S_{p_{i} q_{i}}, 1 \leqslant i \leqslant k_{1}$, содержат отталкивающие неподвижные точки, а шары, приклеенные к сферам $S_{p_{i} q_{i}}$, $k_{1}+1 \leqslant i \leqslant k_{2}$, содержат притягиваюшие неподвижные точки диффеоморфизма $f_{1}$. Продолжение $\left.f\right|_{M_{1}}$ до $f_{1}$ можно произвести таким образом, чтобы $f_{1}$ стал $A$-диффеоморфизмом и имел аттрактор $\Omega^{\prime}$, где $\Omega^{\prime}$ совпадает с $\Omega_{1}$ как множество. Так как локально $\Omega^{\prime}$ гомеоморфно произведению канторова множества и $\mathbb{R}^{n-1}$, то топологическая размерность $\Omega^{\prime}$ равна $n-1$. В то же время, $\Omega^{\prime}$ состоит из $(n-1)$-мерных неустойчивых многообразий своих точек. Следовательно, $\Omega^{\prime}-$ ориентируемый растягивающийся аттрактор коразмерности один.

В силу построения каждая характеристическая сффера аттрактора $\Omega^{\prime}$ ограничивает в $M_{1}^{\prime}$ шар. В силу второго утверждения

$$
M_{1}^{\prime}=\bigcup_{\nu=1}^{k_{2}} \bigcup_{j \geqslant 0} f^{j}\left(E_{p_{\nu} q_{\nu}}\right) \cup \Omega^{\prime}=\bigcup_{\nu}^{k_{2}} E_{p_{\nu} q_{\nu}} \cup W^{s}\left(\Omega^{\prime}\right)
$$

и $\Omega^{\prime}$ есть $C$-плотное базисное множество. Так как

$$
\bigcup_{\nu=1}^{k_{2}} S_{p_{\nu} q_{\nu}}=\bigcup_{\nu=1}^{k_{2}} \partial E_{p_{\nu} q_{\nu}} \subset W^{s}\left(\Omega^{\prime}\right)
$$


то $k_{2}=k_{1}$, в противном случае существовали бы связывающие цилиндры, которые принадлежали бы как $W^{s}\left(\Omega^{\prime}\right)$, так и устойчивым многообразиям соответствующих притягивающих неподвижных точек, что невозможно.

Пусть $A_{p_{\nu} q_{\nu}}=\operatorname{clos}\left(f\left(E_{p_{\nu} q_{\nu}}\right)-E_{p_{\nu} q_{\nu}}\right), 1 \leqslant \nu \leqslant k_{1}$. Из предыдущего доказательства имеем равенство

$$
M_{1}=\bigcup_{\nu=1}^{k_{1}} \bigcup_{j \geqslant 0} f^{j}\left(A_{p_{\nu} q_{\nu}}\right) \cup \Omega^{\prime}
$$

Следовательно, $M_{1}$ является топологическим $n$-мерным подмногообразием многообразия $M^{n}$ с гранищей $\partial D\left(\Omega^{\prime}\right)=\bigcup_{i=1}^{k_{1}} S_{p_{i} q_{i}}$.

Аналогичные соображения применяются к остальным компонентам $M_{i}$, которые содержат целиком, по крайней мере, одно неустойчивое многообразие базисного множества $\Omega$. В частности, если int $M_{i}, 1 \leqslant i \leqslant l$, содержит неустойчивое многообразие $W^{u} \subset \Omega$, принадлежащее $C$-плотной компоненте $\Omega_{c} \subset \Omega$, то любое граничное неустойчивое многообразие из $\Omega_{c}$ лежит в $M_{i}$, поскольку $W^{u}$ всюду плотно в $\Omega_{c}[2]$, [35]. Следовательно, $\Omega_{c, i}=\Omega_{c} \subset M_{i}$, где $\Omega_{c, i}=M_{i} \cap \Omega$. Более того, $\Omega_{c, i}$ - единственная $C$-плотная компонента из $\Omega$, лежащая в $M_{i}$. Очевидно, такие компоненты $M_{i}$ образуют множество $D(\Omega)$, которое является топологическим $n$-мерным подмногообразием многообразия $M^{n}$ с гранищей $\partial D(\Omega)=\bigcup_{i=1}^{k} S_{p_{i}} q_{i}$. Таким образом, из второго утверждения вытекает первое.

Докажем теперь второе утверждение. Сперва докажем равенство

$$
\bigcup_{i=1}^{k_{1}} E_{p_{i} q_{i}} \cup W^{s}\left(\Omega_{c}\right)=\bigcup_{i=1}^{k_{1}} \bigcup_{j \geqslant 0} f^{j}\left(E_{p_{i} q_{i}}\right) \cup \Omega_{c} \stackrel{\text { def }}{=} M_{0}
$$

где $\Omega_{c}$ - некоторая $C$-плотная компонента базисного множества $\Omega$. Предположим, что ассоциированные граничные неподвижные точки из $\Omega_{c}$ образуют семейство $\left\{p_{i}, q_{i}\right\}_{i=1}^{k_{1}}, k_{1} \leqslant k$. Из $(2.2)$ и $(2.3)$ вытекает, что

$$
A_{p_{i} q_{i}}=\operatorname{clos}\left(f\left(E_{p_{i} q_{i}}\right)-E_{p_{i} q_{i}}\right) \subset D_{p_{i} q_{i}}
$$

В силу леммы $2.6 D_{p_{i} q_{i}} \subset W^{s}\left(\Omega_{c}\right)$ для $i \leqslant k_{1}$. Поэтому

$$
\bigcup_{i=1}^{k_{1}} \bigcup_{j \geqslant 0} f^{j}\left(E_{p_{i} q_{i}}\right) \cup \Omega \subset \bigcup_{i=1}^{k_{1}} E_{p_{i} q_{i}} \cup W^{s}\left(\Omega_{c}\right)
$$

Докажем обратноевключение. Возьмем произвольную точку $z \in W^{s}\left(\Omega_{c}\right)-\Omega_{c}$. Согласно предложению 1.4 точка $z$ принадлежит либо дуге $(x, y)_{\varnothing}^{s}$ с $x \in W^{u}\left(p_{i}\right)$ и $y \in W^{u}\left(q_{i}\right)$, либо $z \in W_{\varnothing}^{s}\left(p_{i}\right)$ для некоторой граничной периодической точки $p_{i} \in \Omega_{c}, i \leqslant k_{1}$. В последнем случае $z \in E_{p_{i} q_{i}}$. Для $z \in(x, y)_{\varnothing}^{s}$ рассмотрим две возможности. Если $x \in D_{p_{i}}$, то $z \in E_{p_{i} q_{i}}$. Если $x \notin D_{p_{i}}$, то $z \in D_{p_{i} q_{i}}$. Таким образом, в любом случае $z \in \bigcup_{j \geqslant 0} f^{j}\left(E_{p_{i} q_{i}}\right)$. Поэтому

$$
W^{s}\left(\Omega_{c}\right)-\Omega_{c} \subset \bigcup_{i=1}^{k_{1}} \bigcup_{j \geqslant 0} f^{j}\left(E_{p_{i} q_{i}}\right) .
$$


Теперь покажем, что множество $M_{0}$ открыто и замкнуто. Очевидно, $W^{s}\left(\Omega_{c}\right)$ открыто. Любая характеристическая сфера $S_{p_{i} q_{i}}$ принадлежит $W^{s}\left(\Omega_{c}\right)$, поскольку $\Omega_{c} \subset W^{s}\left(\Omega_{c}\right)$. Следовательно, $M_{0}=\bigcup_{i=1}^{k_{1}} E_{p_{i} q_{i}} \cup W^{s}\left(\Omega_{c}\right)$ открыто.

Возьмем произвольную точку $z_{0} \in \cos M_{0}$. Тогда существует последовательность $z_{s} \in \bigcup_{i=1}^{k_{1}} \bigcup_{j \geqslant 0} f^{j}\left(E_{p_{i} q_{i}}\right) \cup \Omega_{c}$, сходящаяся к $z_{0}$ при $s \rightarrow \infty$. Если имеется бесконечное множество точек $z_{s}$ из $\Omega_{c}$, то $z_{0} \in \Omega_{c} \subset M_{0}$, поскольку $\Omega_{c}$ замкнуто. Поэтому можно считать, что все точки $z_{s}$ не принадлежат $\Omega_{c}$. Тогда, в силу предложения $1.4, z_{s} \in \bigcup_{j \geqslant 0} f^{j}\left(E_{p_{i}} q_{i}\right)-\Omega_{c}$ для некоторого $1 \leqslant i \leqslant k_{1}$. Следовательно, $z_{s} \in f^{j_{s}}\left(E_{p_{i} q_{i}}\right)$ для некоторой последовательности натуральных чисел $j_{s}$. Если эта последовательность $j_{s}$ ограничена, то $z_{0}$ лежит в конечном объединении $n$-шаров вида $f^{l m}\left(E_{p_{i} q_{i}}\right)$, и, следовательно, $z_{0} \in M_{0}$. Если последовательность $j_{s}$ ограничена, то $z_{s} \in\left(x_{s}, y_{s}\right)_{\varnothing}^{s} \subset f^{j_{s}}\left(E_{p_{i} q_{i}}\right)$. Согласно предложению 1.5 $\lambda\left(x_{s}, y_{s}\right)_{\varnothing}^{s} \rightarrow 0$ при $s \rightarrow \infty$, где $\lambda(\cdot, \cdot)$ означает длину дуги $(\cdot, \cdot)$. Поэтому $\lim _{s \rightarrow \infty} z_{s}=\lim _{s \rightarrow \infty} x_{s}$. Так как $x_{s} \in \Omega_{c}$, то $\lim _{s \rightarrow \infty} x_{s} \in \Omega_{c}$. Следовательно, $z_{0}=\lim _{s \rightarrow \infty} z_{s} \in \Omega_{c}$. Это означает, что $M_{0}$ замкнуто. Тогда $M=M_{0}$, поскольку $M$ связно.

Покажем, что $\Omega=\Omega_{c}$. Предположим противное. Тогда $\Omega$ имеет другую $C$-плотную компоненту, скажем $\Omega_{1}$, отличную от $\Omega_{c}$. Так как $M=\bigcup_{i=1}^{k_{1}} E_{p_{i} q_{i}} \cup$ $W^{s}\left(\Omega_{c}\right)$, то $\Omega_{1} \subset \bigcup_{i=1}^{k_{1}} E_{p_{i} q_{i}}$. Из $C$-плотности вытекает, что любое неустойчивое многообразие $W^{u}(x), x \in \Omega_{1}$, всюду плотно в $\Omega_{1}$ [2], [35]. Поэтому $\Omega_{1}-$ связное множество. Следовательно, $\Omega_{1}$ лежит в одном шаре из объединения $\bigcup_{i=1}^{k_{1}} E_{p_{i}} q_{i}$. Для определенности положим $\Omega_{1} \subset E_{p_{1} q_{1}}$. Согласно [2] и [35] $\Omega_{1}$ - растягиваюшийся аттрактор коразмерности один для некоторой итерации $f^{r}, r \in \mathbb{N}$. С другой стороны, Коллмер [50] доказал, что односвязное $n$-многообразие, $n \geqslant 3$, не может содержать гиперболических аттракторов коразмерности один (см. также [60], где доказано, что растягиваюшийся аттрактор коразмерности один $\Lambda$ определяет нетривиальный элемент групшы гомологий, если оба подрасслоения $E_{\Lambda}^{s}$ и $E_{\Lambda}^{u}$ в разложении $T_{\Lambda} M=E_{\Lambda}^{s} \oplus E_{\Lambda}^{u}$ ориентируемы). Противоречие.

СЛЕДСТВИЕ 2.4. Предположим, что выполняются условия теоремы 2.1, пусть имеется другое базисное множество $\Omega^{\prime} \neq \Omega$. Тогда если неустойчивое многообразие $W^{u}\left(z^{\prime}\right)$ некоторой точки $z^{\prime} \in \Omega^{\prime}$ пересекает $D_{p_{i} q_{i}}$, то $W^{u}\left(z^{\prime}\right)$ пересекает связывающий чилиндр $C_{p_{i}} q_{i}$.

ДокАЗАТЕльство. Так как устойчивые многообразия базисных множеств попарно не пересекаются, то $\Omega^{\prime} \cap D_{p_{i} q_{i}}=\varnothing$. Заметим, что $W^{u}\left(z^{\prime}\right)$ связно и не пересекает $W^{u}\left(p_{i}\right) \cup W^{u}\left(q_{i}\right)$. Отсюда и из теоремы 2.1 следует требуемое утверждение.

2.3. Применения теории ламинаций к инвариантным многообразиям. Пусть $\bar{M}-$ универсальное накрывающее многообразие для $M$ и $\pi: \bar{M} \rightarrow M-$ соответствующее накрытие. Группу накрывающих отображений будем отождествлять с фундаментальной группой $\pi_{1}(M)$. Напомним, что точки $\bar{x}, \bar{y} \in \bar{M}$ называются конгруэнтными, если $\pi(\bar{x})=\pi(\bar{y})$. В этом случае существует $\alpha \in \pi_{1}(M)$ такое, что $\alpha(\bar{x})=\bar{y}$.

Для любого диффеоморфизма $f: M \rightarrow M$ существует накрьваюший диффеоморфизм $\bar{f}: \bar{M} \rightarrow \bar{M}, \bar{f} \circ \pi=\pi \circ f$, поскольку $\bar{M}$ односвязно. Если $f$ есть $A$-диффеоморфизм, то $\bar{f}$ имеет инвариантное множество $\pi^{-1}(N W(f))$, наделенное структурой локального произведения [39], [44], [63]. Обозначим через $w^{u(s)}(\bar{x})$ поднятие 
инвариантного многообразия $W^{u(s)}(x), x \in N W(f)$, где $\bar{x} \in \bar{M}$ - поднятие точки $x$. Имеем

$$
\bar{M}=\bigcup_{\bar{x} \in \pi^{-1}(N W(f))} w^{u}(\bar{x})=\bigcup_{\bar{x} \in \pi^{-1}(N W(f))} w^{s}(\bar{x}),
$$

поскольку $M=\bigcup_{x \in N W(f)} W^{u}(x)=\bigcup_{x \in N W(f)} W^{s}(x)$. Так как каждое инвариантное многообразие диффеоморфизма $f$ является инъективно иммерсированным образом евклидового пространства, то каждое $w^{u(s)}(\bar{x})$ также является инъективно иммерсированным образом евклидового пространства. Более того, оба семейства

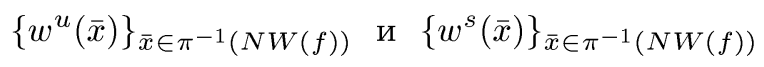

инвариантны относительно $\bar{f}$. Поэтому $w^{u(s)}(\bar{x})$ называется неустойчивым (устойчивым) инвариантным. многообразием $\bar{f}$. Таким образом, $\bar{M}$ представляет собой объединение попарно непересекаюшихся неустойчивых (устойчивых) инвариантных многообразий, которые являются поднятиями неустойчивых (устойчивых) инвариантных многообразий диффеоморфизма $f$.

Предположим, что $f: M \rightarrow M$ имеет ориентируемьй растягивающийся аттрактор (или сжимающийся репеллер) $\Omega$ коразмерности один. Тогда $\bar{\Omega}=\pi^{-1}(\Omega)$ есть инвариантное гиперболическое множество диффеоморфизма $\bar{f}$, состоящее из гладко иммерсированных подмногообразий коразмерности один и локально гомеоморффное произведению $(n-1)$-мерного евклидового пространства $\mathbb{R}^{n-1}$ и канторова множества [39], [44]. Поэтому можно естественным образом ввести понятия граничных точек (необязательно периодических относительно $\bar{f}$ ), граничных многообразий, ассоциированных точек и связок для $\bar{\Omega}$. Из предложения 1.2 и леммы 1.1 вытекает, что граничные точки множества $\bar{\Omega}$ в точности совпадают с прообразом $\pi^{-1}\left(B_{\Omega}\right)$, где $B_{\Omega}-$ множество граничных точек множества $\Omega$.

Далее считаем $\Omega$ растягивающимся аттрактором. Будем придерживаться обозначений п. 2.1.

ЛЕмма 2.9. Пусть $B_{p q}=W^{u}(p) \cup W^{u}(q)$ - связка ориентируемого растягивающегося аттрактора $\Omega$, состоящая из неустойчивых многообразий $W^{u}(p), W^{u}(q)$ ассочиированных граничных периодических точек $p$ и q соответственно, и $\bar{p}$ - поднятие точки $p$. Тогда существует единственное поднятие $\bar{q}$ точки q такое, что $\bar{B}_{\bar{p} \bar{q}}=w^{u}(\bar{p}) \cup w^{u}(\bar{q}) \subset \pi^{-1}\left(B_{p q}\right)$ есть 2-связка множества $\bar{\Omega}$. Более того, существует гомеоморфизм

$$
\bar{\varphi}_{\bar{p} \bar{q}}:\left(w^{u}(\bar{p})-\bar{p}\right) \cup\left(w^{u}(\bar{q})-\bar{q}\right) \rightarrow\left(w^{u}(\bar{p})-\bar{p}\right) \cup\left(w^{u}(\bar{q})-\bar{q}\right),
$$

который является накрывающим для $\varphi_{p q}$ и который продолжается до гомеоморфизма

$$
\bar{\varphi}_{\bar{p} \bar{q}}: w^{u}(\bar{p}) \cup w^{u}(\bar{q}) \rightarrow w^{u}(\bar{p}) \cup w^{u}(\bar{q}),
$$

əде $\bar{\varphi}_{\bar{p} \bar{q}}(\bar{p})=\bar{q} u \bar{\varphi}_{\bar{p} \bar{q}}(\bar{q})=\bar{p}$.

ДокАЗАТЕЛЬСТВо. Возьмем произвольную точку $\bar{x} \in w^{u}(\bar{p})-\bar{p}$. Так как $\pi\left(w^{u}(\bar{p})-\bar{p}\right)=W^{u}(p)-p$, то $x=\pi(\bar{x}) \in W^{u}(p)-p$. Пусть $[\bar{x}, \bar{y}]^{s}-$ поднятие дуги $\left[x, \varphi_{p q}(x)\right]_{\varnothing}^{s}$. Очевидно, $(\bar{x}, \bar{y})^{s} \cap \bar{\Omega}=\varnothing$ и $\bar{y} \in \bar{\Omega}$. Поэтому $(\bar{x}, \bar{y})^{s}=(\bar{x}, \bar{y})_{\varnothing}^{s}$. Из определения граничной точки и предложения 1.2 вытекает существование точки $\bar{q} \in \pi^{-1}\left(B_{\Omega}\right)$. В силу теоремы о накрываюшей гомотопии (см., например, [29, 
гл. 4]) $\bar{q}$ есть поднятие точки $q$. Из ориентируемости $\Omega$ следует, что $\bar{q} \neq \bar{p}$. Точка $\bar{q}$ не зависит от выбора точки $\bar{x} \in w^{u}(\bar{p})-\bar{p}$, и объединение

$$
\bar{B}_{\bar{p} \bar{q}}=w^{u}(\bar{p}) \cup w^{u}(\bar{q}) \subset \pi^{-1}\left(B_{p q}\right)
$$

является 2-связкой множества $\bar{\Omega}$, которая есть поднятие 2 -связки $B_{p q}$.

Таким образом, для любой точки $\bar{x} \in w^{u}(\bar{p})-\bar{p}$ существует единственная точка $\bar{y} \in w^{u}(\bar{q})$ такая, что $(\bar{x}, \bar{y})^{s}=(\bar{x}, \bar{y})_{\varnothing}^{s}$, и наоборот. Положим $\bar{\varphi}_{\bar{p} \bar{q}}(\bar{x})=\bar{y}, \bar{\varphi}_{\bar{p} \bar{q}}(\bar{y})=$ $\bar{x}$. В силу теоремы о накрывающей гомотопии $\bar{\varphi}_{\bar{p} \bar{q}}$ есть поднятие отображения $\varphi_{p q}$. Отсюда следует, что $\bar{\varphi}_{\bar{p} \bar{q}}$ продолжается до гомеоморфизма $\bar{\varphi}_{\bar{p} \bar{q}}: w^{u}(\bar{p}) \cup w^{u}(\bar{q}) \rightarrow$ $w^{u}(\bar{p}) \cup w^{u}(\bar{q})$, если положить $\bar{\varphi}_{\bar{p} \bar{q}}(\bar{p})=\bar{q}$ и $\bar{\varphi}_{\bar{p} \bar{q}}(\bar{q})=\bar{p}$, поскольку $\pi$ является локальным гомеоморфизмом.

СЛЕДСТВИЕ 2.5. Если $B_{p q}-2$-связка множества $\Omega$, mо $\pi^{-1}\left(B_{p q}\right)-$ оббединение попарно непересекающихся 2-связок множества $\bar{\Omega}$. Более того, кажсдая связка из прообраза $\pi^{-1}\left(B_{p q}\right)$ является поднятием $B_{p q}$.

ДоКАЗАТЕЛЬСтво следует из леммы 2.9 и того, что на любом инвариантном неустойчивом многообразии из множества $\bar{\Omega}$ нет конгруэнтных точек.

Лемма 2.10. Пусть $B_{p q}=W^{u}(p) \cup W^{u}(q)$ - 2-связка множества $\Omega$ и $\bar{B}_{\bar{p} \bar{q}}$ накрывающая ее 2-связка множества $\bar{\Omega}$, где $\pi(\bar{p})=p u \pi(\bar{q})=q$. Пусть $S_{p q}-$ характеристическая сфера, соответствующая $B_{p q}$ и состоящая из связывающего иилиндра $C_{p q}$ и двух $(n-1)$-шаров $D_{p}, D_{q}$. Тогда существует характеристическая сфера $\bar{S}_{\bar{p} \bar{q}}$, соответствующ, $\bar{B}_{\bar{p}} \bar{q}$ и состоящая из связивающего иилиндра $\bar{C}_{\bar{p} \bar{q}}$ и двух $(n-1)$-шаров $\bar{D}_{\bar{p}}, \bar{D}_{\bar{q}} \operatorname{ma\kappa ux,~что~} \pi\left(\bar{D}_{\bar{p}}\right)=D_{p}$, $\pi\left(\bar{D}_{\bar{q}}\right)=D_{q}$ и $\pi\left(\bar{C}_{\bar{p} \bar{q}}\right)=C_{p q}$. Более того, $\pi^{-1}\left(S_{p q}\right)$ есть облединение попарно непересекающихся характеристических сфер, взаимно однозначно соответствующих связкам из $\pi^{-1}\left(B_{p q}\right)$.

ДокАЗАтЕльство. В силу теоремы о накрывающей гомотопии существуют $(n-1)$-шары $\bar{D}_{\bar{p}} \subset w^{u}(\bar{p}), \bar{D}_{\bar{q}} \subset w^{u}(\bar{q})$ такие, что $\bar{p} \in \bar{D}_{\bar{p}}, \bar{q} \in \bar{D}_{\bar{q}}, \bar{D}_{\bar{p}}$ есть поднятие $D_{p}$ и $\bar{D}_{\bar{q}}$ есть поднятие $D_{q}$. Согласно лемме 2.9

$$
\bar{\varphi}_{\bar{p} \bar{q}}\left(\bar{D}_{\bar{p}}\right)=\bar{D}_{\bar{q}}, \quad \bar{\varphi}_{\bar{p} \bar{q}}\left(\bar{D}_{\bar{q}}\right)=\bar{D}_{\bar{p}}
$$

Следовательно, множество $\bar{C}_{\bar{p} \bar{q}} \stackrel{\text { def }}{=} \bigcup_{\bar{x} \in \partial \bar{D}_{\bar{p}}}\left(\bar{x}, \bar{\varphi}_{\bar{p} \bar{q}}(\bar{x})\right)_{\varnothing}^{s}$ гомеоморфно открытому $(n-1)$-мерному цилиндру $\Sigma^{n-2} \times(0,1)$ и $\pi\left(\bar{C}_{\bar{p} \bar{q}}\right)=C_{p q}$. Поэтому объединение

$$
\bar{S}_{\bar{p} \bar{q}}=\bar{D}_{\bar{p}} \cup \bar{D}_{\bar{q}} \bigcup_{\bar{x} \in \partial \bar{D}_{\bar{p}}}(\bar{x}, \bar{y})_{\varnothing}^{s}
$$

есть характеристическая сфера, соответствующая связке $\bar{B}_{\bar{p}}$.

Так как инвариантное многообразие любой точки из $\bar{\Omega}$ не имеет конгруэнтных точек, то ограничение $\pi$ на каждой характеристической сфере из $\bar{\Omega}$ инъективно. Это завершает доказательство.

Далее мы предполагаем знакомство читателя с основами теории слоений и ламинаций. Достаточным источником может служить книга [31] или статья [54]. Напомним определение ламинации. 
Пусть $\mathscr{S} \subset M^{n}$-некоторое подмножество многообразия $M^{n} ; d$-мерной локальной $C^{r, l}$-ламиначией $\mathscr{L}, 0 \leqslant l \leqslant r \leqslant \infty$, заданной на множестве $\mathscr{S} \stackrel{\text { def }}{=} \operatorname{supp} \mathscr{L}$, называется разбиение $\mathscr{S}$ на попарно непересекающиеся линейно связные подмножества $L_{\alpha}, \operatorname{supp} \mathscr{L}=\bigcup_{\alpha} L_{\alpha}$, называемые слоями, такое, что выполняется следующее свойство: для любой точки $x \in \operatorname{supp} \mathscr{L}$ сушествуют окрестность $U(x) \subset M^{n}$, $x \in U(x)$, и $C^{l}$-диффеоморфизм $\varphi: U(x) \rightarrow \mathbb{R}^{n}$ такие, что любая связная компонента пересечения $U(x) \cap L_{\alpha}$ (если это пересечение не пусто) отображается $C^{l}$-диффеоморфизмом $\varphi$ в $d$-мерную гиперплоскость вида $x_{d+1}=c_{1}, \ldots, x_{n}=$ $c_{n-d}$, при этом ограничение $\left.\varphi\right|_{U(x) \cap L_{\alpha}}$ является $C^{r}$-диффеоморфизмом на образ. Если подмножество supp $\mathscr{L}$ замкнуто, то $\mathscr{L}$ называется $d$-мерной $C^{r, l}$-ламинаиией. Если $d=n-1$, то $\mathscr{L}$ называется ламинаиией коразмерности один.

Допуская некоторую вольность, можно сказать, что локальная ламинация есть слоение без особенностей на некотором подмножестве. Если это подмножество совпадает с многообразием, $\mathscr{L}=M^{n}$, то локальная $C^{r, l}$-ламинация является $C^{l}$-слоением. Ламинация есть слоение без особенностей на замкнутом подмножестве.

ЛЕмма 2.11. Пусть $\mathscr{L}-d$-мерная $C^{r, l}$-ламинация и $L_{\alpha}-$ слой ламинаиии $\mathscr{L}$. Пусть $X$ - компактное подмножество слоя $L_{\alpha}$. Тогда существуют окрестность $U(X) \subset M$ множсества $X \subset U(X)$ и $C^{l}$-диффеоморфизм $\varphi_{X}: U(X) \rightarrow \mathbb{R}^{n}$ такие, что для любого слоя $L_{\beta}$ каждая компонента пересечения $U(X) \cap L_{\beta}$ (локальный слой в $U(X)$ ) отображается посредством $\varphi_{X}$ в d-мерную гиперплоскость $x_{d+1}=c_{1}, \ldots, x_{n}=c_{n-d}$ для некоторьх $c_{1}, \ldots, c_{n-d}$.

ДоказАТЕльство. Имеется конечное покрытие компактного множества $X$ картами $\left\{U\left(x_{i}\right), \varphi_{i}\right\}$, в которых ламинация $\mathscr{L}$ устроена как семейство параллельных гиперплоскостей. Далее, аналогично доказательству теоремы 5.1 из [31], строится конечное семейство когерентных карт, покрывающих $X$. Отсюда вытекает требуемьй результат.

СЛЕДСТВИЕ 2.6. Предположим, что выполняются условия леммы 2.11. Тогда существуют окрестность $U(X) \subset M, X \subset U(X)$, и d-мерное $C^{l}$-слоение $\mathscr{F}$ в $U(X)$ такие, что локальные слои ламинации $\mathscr{L}$ в $U(X)$ являются слоями $\mathscr{F}$.

Фактически следствие 2.6 означает, что ламинация вблизи компактного множества на слое может быть вложена в слоение.

ЛЕмма 2.12. Пусть $\mathscr{L}-C^{r, l}$-ламиначия коразмерности один, $1 \leqslant l \leqslant r$, u $L_{\alpha}-$ односвязный слой ламиначии $\mathscr{L}$. Тогда группа голономии слоя $L_{\alpha}$ тривиальна.

ДокАЗАтЕльство. Рассмотрим точку $x_{0} \in L_{\alpha}$ и гладкую замкнутую петлю $\gamma \subset L_{\alpha}$ в $x_{0}$. Пусть $l$ - трансверсальный $\mathscr{L}$ отрезок, проходяший через $x_{0}$. Тогда $\mathscr{L}$ индуцирует отображение $G_{\gamma}: l \cap \mathscr{L} \rightarrow l \cap \mathscr{L}$, соответствующее петле $\gamma$. Это отображение определяет элемент $g_{\gamma}$ группы голономии. Так как $L_{\alpha}$ односвязный, то $\gamma$ стягиваема, и мы можем считать, что $\gamma$ ограничивает 2 -диск $D \subset L_{\alpha}$, поскольку $L_{\alpha}-$ гладко иммерсированное многообразие коразмерности один. В силу следствия 2.6 существует окрестность $U(D)$ диска $D$ такая, что $\mathscr{L}$ вкладывается в $C^{l}$-слоение. Теорема Рэба (Reeb) об устойчивости односвязного слоя [61] влечет, 
что $G_{\gamma}=$ id (см. также [31, теоремы $\left.5.7,5.12\right]$ ). Следовательно, $g_{\gamma}=$ id. Это завершает доказательство.

Пусть $\mathscr{L}-C^{r, l}$-ламинация, $1 \leqslant l \leqslant r$, коразмерности один такая, что каждый ее слой наделен нормальной ориентацией, и пусть $T$ - дуга или замкнутая кривая, наделенная некоторой внутренней ориентацией. Будем говорить, что $T$ пересекает слой $L_{\alpha}$ ламинации $\mathscr{L}$ ориентируемо, если индекс пересечения в каждой точке из $L_{\alpha} \cap T$ одинаков (либо +1 , либо -1$)$.

Пусть $f: M \rightarrow M-A$-диффеоморфизм, имеюший ориентируемый растягиваюшийся аттрактор $\Omega$. Тогда неустойчивые многообразия точек из $\Omega$ образуют $C^{\infty, 1}$-ламинацию коразмерности один, которую мы обозначим через $\mathscr{L}(\Omega)[44]$,

$$
\mathscr{L}(\Omega)=\left\{W^{u}(x)\right\}_{x \in \Omega}, \quad \operatorname{supp} \mathscr{L}(\Omega)=\bigcup_{x \in \Omega} W^{u}(x) .
$$

Следовательно, $\pi^{-1}(\mathscr{L}(\Omega))=\mathscr{L}(\bar{\Omega})$ есть $C^{\infty, 1}$-ламинация на $\bar{M}$, состоящая из неустойчивых многообразий точек из $\bar{\Omega}$, поскольку $\pi$ - локальньй диффеоморфизм.

Напомним, что каждое неустойчивое многообразие растягивающегося аттрактора $\Omega$ является гладкой иммерсией евклидового пространства $\mathbb{R}^{n-1}$. Поэтому каждый слой ламинации $\mathscr{L}(\Omega)$ может быть наделен нормальной ориентацией.

ЛЕмма 2.13. Пусть $T \subset M-$ замкнутая простая кривая, пересекающая слои ламиначии $\mathscr{L}(\Omega)$ трансверсально и ориентируемо. Тогда $T$ негомотопна нулю в $M$.

ДокАЗАТЕЛЬСтво. Предположим противное. Тогда существует гладкая иммерсия $\psi: D^{2} \rightarrow M$ двумерного диска $D^{2}$ в $M$ такая, что $\psi\left(\partial D^{2}\right)=T$ (см., например, [31, гл. 6]). Так как пересечение $\mathscr{L}(\Omega) \cap \psi\left(D^{2}\right)$ компактно, то его можно покрыть конечным числом окрестностей $\left\{U_{i}\right\}_{i=1}^{k}$, в каждой из которых ламинация $\mathscr{L}(\Omega)$ устроена как семейство параллельных гиперплоскостей. В силу леммы 2.11 $\operatorname{supp} \mathscr{L}(\Omega) \cap U_{i}$ доопределяется до $C^{1}$-слоения $\mathscr{F}_{i}$ для каждого $1 \leqslant i \leqslant k$. Поэтому в каждой окрестности $U_{i}$ можно применить стандартную технику, развитую в лемме 5.1 из [39], приведения диска $\psi\left(D^{2}\right)$ в общее положение относительно слоения $\mathscr{F}_{i}$. Это означает, что пересечение диска $\psi\left(D^{2}\right)$ со слоями является семейством одномерных кривых на $\psi\left(D^{2}\right)$ с конечным числом особенностей, в которых диск $\psi\left(D^{2}\right)$ касается слоев слоения $\mathscr{F}_{i}$ (см. подробное описание этой техники в примечании Д. В. Аносова в книге [31, с. 281-285] для $C^{1}$-слоений). В силу того, что в каждой окрестности $U_{i}$ возникает только конечное число точек касания, можно на каждом шаге производить шевеление диска, не меняя предыдуших шевелений возле ранее получившихся точек касания. Поэтому отображение $\psi$ можно изменить таким образом, чтобы диск $\psi\left(D^{2}\right)$ находился в общем положении относительно ламинации $\mathscr{L}(\Omega)$, т.е. пересечение диска $\psi\left(D^{2}\right)$ со слоями ламинации $\mathscr{L}(\Omega)$ есть семейство одномерных кривых на $\psi\left(D^{2}\right)$ с конечным числом особенностей, в которых диск $\psi\left(D^{2}\right)$ касается слоев ламинации.

Окружим теперь каждую точку касания попарно непересекающимися окрестностями, в каждой из которых $\mathscr{L}(\Omega)$ доопределяется до некоторого $C^{1}$-слоения. В силу [39] можно изменить отображение $\psi$ в этих окрестностях так, чтобы диск $\psi\left(D^{2}\right)$ имел точки касания только морсовского типа. Обозначим через $\mathscr{F} D^{2}$ одномерную ламинацию, которая индуцируется на диске $D^{2}$ ламинацией $\mathscr{L}(\Omega)$, посредством отображения $\psi^{-1}$. Так как $\psi\left(D^{2}\right)$ имеет точки касания со слоями ламинации 
$\mathscr{L}(\Omega)$ только морсовского типа, то $\mathscr{F}_{D^{2}}$ имеет только изолированные особенности типа "центр" и "седло". Более того, в силу [39] можно считать, что слоение $\mathscr{F}_{D^{2}}$ трансверсально гранище $\partial D^{2}$ и не имеет сепаратрис, соединяющих разные седла. Отметим, что в упомянутом примечании Д.В. Аносова в книге [31, с. 281-285] приводится доказательство этих фактов, включая существование $C^{1}$-иммерсии $\psi: D^{2} \rightarrow M$.

По предположению $\mathscr{L}(\Omega) \cap T \neq \varnothing$. Возьмем слой $l$ слоения $\mathscr{F}_{D^{2}}$, проходящий через точку $x_{0} \in \partial D^{2}$ такую, что $\psi\left(x_{0}\right) \in \mathscr{L}(\Omega) \cap T$. Ориентируем $l$ так, чтобы он входил внутрь $D^{2}$ при движении в положительном направлении. Так как пересечение $\mathscr{L}(\Omega) \cap T$ канторово (см. предложение 1.5 ), то можно считать, что $l$ не является сепаратрисой "седла", в противном случае можно взять близкий слой с данным свойством. Из ориентируемости пересечения $T$ с $\mathscr{L}(\Omega)$ следует, что $l$ при движении в положительном направлении не пересекает границу $\partial D^{2}$. Так как множество $\mathscr{L}(\Omega) \cap \psi\left(D^{2}\right)$ замкнуто, то предельное множество $\omega(l)$ слоя $l$ непусто и состоит из цельх слоев слоения $\mathscr{F}_{D^{2}}$, также не пересекающих $\partial D^{2}$. Множество $\omega(l)$ содержит, по крайней мере, один одномерный слой, скажем $l_{0}$, потому что слоение $\mathscr{F} D^{2}$ имеет особенности только типа "центр" и "седло". Из теории Пуанкаре-Бендиксона для слоений и ламинаций с конечным числом особенностей [6] вытекает, что $l_{0}$ не может содержать в своем предельном множестве незамкнутые одномерные слои, поскольку индексы центра и седла являются цельми числами. Тогда $l_{0}$ - либо замкнутый слой, либо петля сепаратрисы (напомним, что $\mathscr{F} D^{2}$ не имеет сепаратрис, соединяющих разные седла), образующая односторонний контур. Так как $\mathscr{L}(\Omega)$ - замкнутое множество, то $l_{0}$ принадлежит слою $L_{0}$ этой ламинации. Из того, что $l_{0}$ лежит в предельном множестве незамкнутого слоя $l$, следует, что $L_{0}$ имеет нетривиальную группу голономии. С другой стороны, все слои ламинации $\mathscr{L}(\Omega)$ односвязны и согласно лемме 2.12 имеют тривиальную группу голономии. Противоречие.

СЛЕДСТВИЕ 2.7. Пусть простая замкнутая кривая $C$ состоит из дуги, лежащей в $W^{u}(x), \quad x \in \Omega$, и отрезка устойчивого многообразия $W^{s}(x)$, принадлезсащего окрестности точки $x$ с локальной структурой произведения. Тогда кривая $C$ не гомотопна нулю в $M$.

ДокАЗАТЕЛЬСТво. Так как в силу ориентируемости $\Omega$ отрезок устойчивого многообразия $W^{s}(x)$ пересекает слои ламинации $\mathscr{L}(\Omega)$ ориентируемо, то стандартной процедурой из теории динамических систем (см., например, [33]) можно пошевелить отрезок устойчивого многообразия $W^{s}(x)$ в окрестности точки $x$ так, чтобы получить замкнутую кривую, которая ориентируемо пересекает слои ламинации $\mathscr{L}(\Omega)$ и гомотопна $C$. Требуемьй результат теперь следует из леммы 2.13.

СлЕДСТВИЕ 2.8. Пусть $\Omega^{\prime}$ - ориентируемый растягивающийся аттрактор или сжимающийся репеллер коразмерности один $A$-диффеоморфизма $f: M \rightarrow$ $M$. Тогда не существует шара $E \subset M$ такого, ито $\Omega^{\prime} \subset E$.

ДокАЗАТЕЛЬСТво. Предположим противное. Не уменьшая обшности, можно считать, что $\Omega^{\prime} C$-плотное. В силу следствия 2.7 существует простая замкнутая кривая $C \subset E$, которая не гомотопна нулю в $M$. Это противоречит гомотопности нулю этой кривой в $E$. 
СлЕДСТВИЕ 2.9. Пусть $\Omega$ - ориентируемый растягивающийся аттрактор коразмерности один $A$-диффеоморфизма $f: M \rightarrow M$, и пусть $\left\{p_{i}, q_{i}\right\}_{i=1}^{k}-n a-$ ры ассочиированных граничных периодических точек $\Omega$. Если все характеристические сферы $S_{p_{i} q_{i}}$ множества $\Omega$ ограничивают шар в $M$, то $\Omega$ является единственным псевдоручным (т.е. растягивающимся аттрактором или сжимающимся репеллером) ориентируемым базисным множеством коразмерности один диффеоморфизма $f$.

ДоКАЗАТЕЛЬСТво. Предположим противное. Пусть $\Omega^{\prime}$ - ориентируемое псевдоручное базисное множество коразмерности один, отличное от $\Omega$. В силу п. 2 ) теоремы $2.1 \Omega^{\prime}$ лежит внутри некоторого шара $E_{p_{i} q_{i}}$, ограниченного характеристической сферой $S_{p_{i} q_{i}}$, поскольку устойчивые многообразия $W^{s}\left(\Omega^{\prime}\right), W^{s}(\Omega)$ попарно не пересекаются. Это противоречит следствию 2.8 .

\section{§3. Гомеоморфность накрывающей пространству $\mathbb{R}^{n}$}

Везде в этом параграфе $\Omega$ - ориентируемый растягиваюшийся аттрактор коразмерности один $A$-диффеоморфизма $f: M \rightarrow M$ и $\bar{f}: \bar{M} \rightarrow \bar{M}$ - поднятие $f$ на универсальную накрывающую относительно накрытия $\pi: \bar{M} \rightarrow M$.

Лемма 3.1. Пусть $\bar{\Omega}=\pi^{-1}(\Omega)$. Тогда:

1) для любой точки $\bar{x} \in \bar{\Omega}$ отображение включения $w^{u}(\bar{x}) \subset \bar{M}$ является вложением;

2) для любой точки $\bar{x} \in \bar{\Omega}$ отображение включения $w^{u}(\bar{x}) \subset \bar{M}$ является собственным отображением ${ }^{1}$, т.е. для любого компакта $K \subset \bar{M}$ пересечение $K \cap w^{u}(\bar{x})$ компактно в топологии неустойчивого многообразия $w^{u}(\bar{x})$;

3) для любой точки $\bar{x} \in \bar{\Omega}$ множество $\bar{M}-w^{u}(\bar{x})$ состоит из двух компонент (другими словами, любой слой $w^{u}(\bar{x})$ разбивает $\left.\bar{M}\right)$;

4) для любых точек $\bar{x}, \bar{y} \in \bar{\Omega}$ пересечение $w^{u}(\bar{x}) \cap w^{s}(\bar{y})$ содержит не более одной точки.

ДокАЗАТЕЛЬСТво. Так как отображение включения инъективно, то для доказательства первого утверждения осталось показать, что внутренняя топология неустойчивого многообразия $w^{u}(\bar{x})$ совпадает с топологией, индуцируемой на $w^{u}(\bar{x})$ как на подмножестве $\bar{M}$. Предположим, что первое утверждение не верно. Тогда в достаточно малой окрестности $U\left(\bar{x}_{0}\right)$ некоторой точки $\bar{x}_{0} \in w^{u}(\bar{x})$ со структурой произведения найдутся точки $\bar{x}_{1}, \bar{x}_{2} \in w^{u}(\bar{x})$, принадлежашие различным компонентам пересечения $U \cap w^{u}(\bar{x})$. Поэтому сушествует простая замкнутая кривая $\bar{C}$, состоящая из дуги, лежащей на слое $w^{u}(\bar{x})$, и отрезка $\bar{S} \subset w^{s}(\bar{x})$ такого, что $\pi(\bar{S})$ принадлежит окрестности $\pi\left(U\left(\bar{x}_{0}\right)\right)$ точки $\pi(\bar{x})$ со структурой произведения. Так как $\bar{C}$ гомотопна нулю в $\bar{M}$, то $\pi(\bar{C})$ гомотопна нулю в $M$, что противоречит следствию 2.7.

Докажем второе утверждение. Предположим противное. Тогда существует последовательность точек $\bar{z}_{k} \in w^{u}(\bar{x})$, не ограниченная в топологии слоя $w^{u}(\bar{x})$, такая, что $\bar{z}_{k} \rightarrow z \in K$ при $k \rightarrow \infty$. Так как неустойчивые многообразия из $\bar{\Omega}$ образуют ламинацию коразмерности один, то $\bar{z} \in \bar{\Omega}$ и существует окрестность $U(\bar{z})$ точки $\bar{z}$, в которой ламинация $\mathscr{L}(\bar{\Omega})$ гомеоморфна семейству параллельных

\footnotetext{
${ }^{1}$ Отметим, что второе утверждение не следует автоматически из первого, поскольку $w^{u}(\bar{x})$ некомпактно в $\bar{M}$.
} 
гиперплоскостей коразмерности один. Не уменьшая общности, можно считать, что в $U(\bar{z})$ имеется локальная структура произведения. Обозначим через $\bar{l}_{k}$ локальные слои (т.е. компоненты пересечения $\left.w^{u}(\bar{x}) \cap U(\bar{z})\right)$, содержащие точки $\bar{z}_{k}$. Поскольку точки $\bar{z}_{k} \in w^{u}(\bar{x})$ образуют неограниченную последовательность в топологии слоя $w^{u}(\bar{x})$, они не могут лежать в одном локальном слое $\bar{l}_{k_{0}}$. Следовательно, имеется, по крайней мере, два локальных слоя $l_{k_{1}} \neq l_{k_{2}}$. Далее получаем противоречие, полностью аналогичное доказательству первого утверждения. Именно, из $l_{k_{1}} \neq l_{k_{2}}$ вытекает, что существует простая замкнутая кривая $\bar{C}$, состоящая из дуги, лежащей на слое $w^{u}(\bar{x})$, и отрезка $\bar{S} \subset w^{s}(\bar{x})$ такого, что $\pi(\bar{S})$ принадлежит окрестности точки $\pi(\bar{x})$ со структурой произведения. Это противоречит следствию 2.7.

Для доказательства третьего утверждения мы воспользуемся рассуждениями Д. В. Аносова, приведенными в [39, примечание к доказательству леммы 5.2]. Проведем через какую-нибудь точку $\bar{y}$ слоя $w^{u}(\bar{x})$ дугу $a$, трансверсально пересекающую этот слой и лежащую в окрестности со структурой произведения. В силу первого утверждения дугу $a$ можно взять столь малой, чтобы она пересекалась $\mathrm{c}$ $w^{u}(\bar{x})$ ровно в одной точке. Нужно доказать, что в $\bar{M}$ не сушествует пути, который соединял бы конщевые точки дуги $a$ и не пересекал бы $w^{u}(\bar{x})$. Предположим, что такой путь существует. Вместе с $a$ он образует замкнутую кривую, которую можно сгладить и малым шевелением избавить от самопересечений. В результате получим простую замкнутую кривую $\bar{C}$, трансверсально пересекаюшую слой $w^{u}(\bar{x})$ ровно в одной точке. Так как $\bar{M}$ односвязно, то на $\bar{C}$ можно натянуть диск $d$, гладко иммерсированный в $\bar{M}$. Путем малого возмущения, ничего не меняющего возле $\bar{C}$, можно достичь того, чтобы во всех точках, где диск пересекает $w^{u}(\bar{x})$, это пересечение было трансверсальным. В силу первого и второго утверждений пересечение $d \cap w^{u}(\bar{x})$ компактно в топологии $w^{u}(\bar{x})$ и состоит из конечного множества кривых, каждая из которых либо замкнута, либо соединяет две точки, лежашие на $\bar{C}$. Так как по построению граница диска пересекается с $w^{u}(\bar{x})$ в точке $\bar{y}$, то пересечение $d \cap w^{u}(\bar{x})$ содержит, по крайней мере, одну дугу, не являющуюся замкнутой кривой и пересекающую гранищу диска в двух различных точках, одна из которых есть точка $\bar{y}$. Это противоречит предположению о том, что точка $\bar{y}$ является единственной точкой пересечения кривой $\bar{C}$ с многообразием $w^{u}(\bar{x})$.

Утверждение 4) следует из ориентируемости аттрактора $\Omega$ и из доказанного выше утверждения 3 ).

Напомним, что в силу леммы 2.9 для каждой граничной точки $\bar{p} \in \bar{\Omega}$ имеется ассоциированная с ней граничная точка $\bar{q} \in \bar{\Omega}$. Пусть $\bar{x} \in \bar{\Omega}$. Положим

$$
w^{s *}(\bar{x})=\left\{\begin{array}{cl}
w^{s}(\bar{x}), & \text { если } w^{s}(\bar{x}) \text { не содержит граничных точек } \bar{\Omega}, \\
w^{s}(\bar{p}) \cup w^{s}(\bar{q}), & \text { если } w^{s}(\bar{x}) \text { содержит граничную точку } \bar{p},
\end{array}\right.
$$

где $\bar{q}$ - точка, ассоциированная $\bar{p}$. Следуюшая лемма обобщает п. 4) леммы 3.1.

ЛЕмма 3.2. Пусть $\Omega$ - ориентируемый растягивающийся аттрактор коразмерности один и $\bar{\Omega}=\pi^{-1}(\Omega)$. Тогда для любых точек $\bar{x}, \bar{y} \in \bar{\Omega}$ пересечение $w^{u}(\bar{x}) \cap w^{s *}(\bar{y})$ содержит не более одной точки.

ДокАЗАТЕЛЬСТво. Предположим противное. Тогда существуют точки $\bar{x}, \bar{y} \in$ $\bar{\Omega}$ такие, что пересечение $w^{u}(\bar{x}) \cap w^{s *}(\bar{y})$ содержит, по крайней мере, две точки 


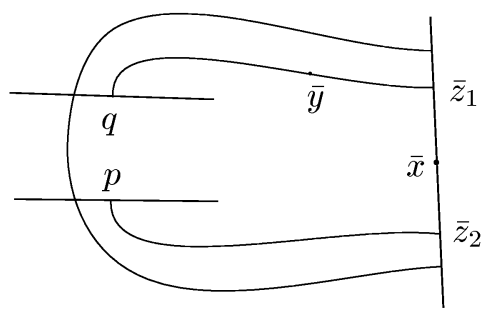

Рис. 6. Пересечение $w^{u}(\bar{x}) \cap w^{s *}(\bar{y})$

$\bar{z}_{1}, \bar{z}_{2}$ (см. рис. 6$)$. В силу леммы $3.1 w^{s *}(\bar{y})$ содержит ассоциированные граничные точки $\bar{p}, \bar{q}$ такие, что $\left.w^{s *(} \bar{y}\right)=w^{s}(\bar{p}) \cup w^{s}(\bar{q})$ и $w^{u}(\bar{x}) \cap w^{s}(\bar{p}) \neq \varnothing, w^{u}(\bar{x}) \cap$ $w^{s}(\bar{q}) \neq \varnothing$. Так как $\Omega$ имеет конечное число граничных точек, то найдется точка $\bar{a} \in w^{u}(\bar{x})$, произвольно близкая к $\bar{z}_{1}$, такая, что $w^{s}(\bar{a})$ не содержит граничных точек из $\bar{\Omega}$. Из теоремы о непрерывной зависимости инвариантных многообразий от начальных условий вытекает, что $w^{s}(\bar{a})$ пересекает $w^{u}(\bar{x})$ вблизи точки $\bar{z}_{2}$ (см. рис. 6). Это противоречит лемме 3.1.

ЛЕмма 3.3. Пусть $\Omega-C$-плотный ориентируемый растягивающийся аттрактор коразмерности один и $\bar{\Omega}=\pi^{-1}(\Omega)$. Предположим, что имеются точки $\bar{x}, \bar{y}, \bar{z} \in \bar{\Omega}$, для которых выполняются следующие условия:

1) $\bar{y} \in w^{u}(\bar{x}), \quad \bar{z} \in w^{s}(\bar{x})$;

2) существует последовательность точек $\bar{x}_{i} \in[\bar{x}, \bar{z})^{s} \cap \bar{\Omega}$ такая, что $\bar{x}_{i} \rightarrow \bar{z}$ при $i \rightarrow \infty$;

3) пересечение $w^{u}\left(\bar{x}_{i}\right) \cap w^{s}(\bar{y})$ состоит из одной точки $\bar{y}_{i}$ для каждого $i \in \mathbb{N}$.

Тогда последовательность точек $\bar{y}_{i}$ ограничена на $w^{s}(\bar{y})$.

ДокАЗАТЕЛЬСтво. Следуем доказательству леммы 5.2 из [39], в которой аналогичный факт доказан для инвариантных многообразий диффеоморфизмов Аносова коразмерности один (как указывает Френкс [39], идея доказательства принадлежит С.П. Новикову).

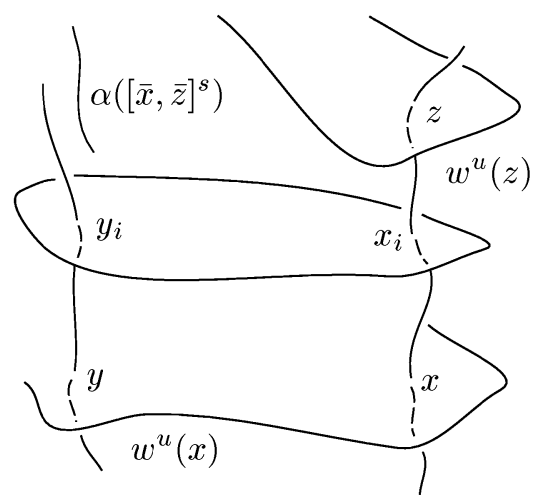

Рис. 7. Последовательность точек $\bar{y}_{i}$ 
В силу леммы 3.1 множество $\bar{M}-w^{u}(\bar{x})$ состоит из двух компонент. Обозначим через $w^{u+}(\bar{x})$ ту компоненту, которая содержит точку $\bar{z}$. Согласно лемме 3.1 $[\bar{x}, \bar{z}]^{s} \subset w^{u+}(\bar{x})$ и, следовательно, все точки $\bar{x}_{i}, \bar{y}_{i}$ принадлежат $w^{u+}(\bar{x})$. Поэтому все $\bar{y}_{i}$ принадлежат одной компоненте множества $w^{s}(\bar{y})-\bar{y}$, которую мы обозначим через $w^{s+}(\bar{y})$ (см. рис. 7). Предположим, что лемма неверна. Тогда последовательность $\bar{y}_{i}$ неограничена на $w^{s+}(\bar{y})$. Поэтому последовательность $\pi\left(\bar{y}_{i}\right)$ неограничена во внутренней топологии кривой $\pi\left(w^{s+}(\bar{y})\right)$. Из предложения 1.3 и $C$-плотности базисного множества $\Omega$ вытекает, что $\pi\left(w^{s+}(\bar{y})\right) \cap \Omega$ всюду плотно в $\Omega$. Следовательно, $\bigcup_{\eta \in \pi_{1}(M)} \eta\left(w^{s+}(\bar{y})\right)$ всюду плотно в $\bar{\Omega}$. Так как $\bar{x} \in \bar{\Omega}$, то существует преобразование $\alpha \in \pi_{1}(M)$, переводящее отрезок $[\bar{x}, \bar{z}]^{s}$ в столь близкий к $w^{s+}(\bar{y})$ отрезок $\alpha\left([\bar{x}, \bar{z}]^{s}\right)$, что любое неустойчивое многообразие из $\bar{\Omega}$, пересекающее $w^{s+}(\bar{y})$, пересекает $\alpha\left([\bar{x}, \bar{z}]^{s}\right)$.

Покажем, что каждое неустойчивое многообразие из $\bar{\Omega}$, которое пересекает отрезок $\alpha\left([\bar{x}, \bar{z}]^{s}\right)$, пересекает также $[\bar{x}, \bar{z}]^{s}$. Возьмем произвольную точку $\bar{a} \in \bar{\Omega} \cap$ $\alpha\left([\bar{x}, \bar{z}]^{s}\right)$. Тогда $w^{u}(\bar{a}) \cap w^{s+}(\bar{y}) \neq \varnothing$. Так как последовательность точек $\bar{y}_{i}$ неограничена на $w^{s}(\bar{y})$, то найдется индекс $j$ такой, что точка $w^{u}(\bar{a}) \cap w^{s+}(\bar{y})$ принадлежит дуге $\left(\bar{y}_{j}, \bar{y}_{j+1}\right)^{s}$. Поэтому точки $\bar{y}_{j}, \bar{y}_{j+1}$ лежат в разных компонентах множества $\bar{M}-w^{u}(\bar{a})$. Следовательно, точки

$$
\bar{x}_{j}=w^{u}\left(\bar{y}_{j}\right) \cap[\bar{x}, \bar{z}]^{s}, \quad \bar{x}_{j+1}=w^{u}\left(\bar{y}_{j+1}\right) \cap[\bar{x}, \bar{z}]^{s}
$$

также лежат в разных компонентах множества $\bar{M}-w^{u}(\bar{a})$. Поэтому неустойчивое многообразие $w^{u}(\bar{a})$ пересекает отрезок $[\bar{x}, \bar{z}]^{s}$ между точками $\bar{x}_{j}, \bar{x}_{j+1}$. Таким образом, каждое неустойчивое многообразие из $\bar{\Omega}$, пересекающее $\alpha\left([\bar{x}, \bar{z}]^{s}\right)$, пересекает также $[\bar{x}, \bar{z}]^{s}$. Это позволяет определить отображение

$$
\lambda \circ \alpha:[\bar{x}, \bar{z}]^{s} \cap \bar{\Omega} \rightarrow[\bar{x}, \bar{z}]^{s} \cap \bar{\Omega},
$$

положив $\lambda(\bar{a})=[\bar{x}, \bar{z}]^{s} \cap w^{u}(\bar{a})$ для $\bar{a} \in \alpha\left([\bar{x}, \bar{z}]^{s}\right) \cap \bar{\Omega}$. Из выбора точки $\bar{z}$ и очевидного равенства $\alpha(\bar{\Omega})=\bar{\Omega}$ вытекает, что $[\bar{x}, \bar{z})^{s} \cap \bar{\Omega} \neq \varnothing$. Поэтому отображение $\lambda \circ \alpha$ имеет непустую область определения. Введем на отрезке $[\bar{x}, \bar{z})^{s}$ ориентацию от точки $\bar{x}$ к точке $\bar{z}$. В силу п. 3 ) леммы 3.1 отображение $\lambda \circ \alpha$ является монотонным на множестве $[\bar{x}, \bar{z}]^{s} \cap \bar{\Omega}$. Поэтому $\lambda \circ \alpha$ может быть продолжено до непрерывного отображения на весь отрезок $[\bar{x}, \bar{z}]^{s}$, поскольку пересечение $[\bar{x}, \bar{z}]^{s} \cap \bar{\Omega}$ является канторовым множеством. Следовательно, отображение $\lambda \circ \alpha$ имеет неподвижную точку $\bar{x}_{0}$. Не уменьшая общности, можно считать, что $\bar{x}_{0} \in \Omega$. Действительно, если $\bar{x}_{0} \notin \Omega$, то $\bar{x}_{0}$ принадлежит некоторому смежному интервалу канторова множества $[\bar{x}, \bar{z}]^{s} \cap \bar{\Omega}$. Тогда в силу монотонности отображения $\lambda \circ \alpha$ и инвариантности $[\bar{x}, \bar{z}]^{s} \cap \bar{\Omega}$ относительно $\lambda \circ \alpha$ конщы этого смежного интервала также являются его неподвижными точками. Из $\alpha(\bar{x})=\bar{x}_{0} \in \Omega$ следует $\alpha\left(w^{u}\left(\bar{x}_{0}\right)\right)=w^{u}\left(\bar{x}_{0}\right)$, что противоречит отсутствию на $w^{u}\left(\bar{x}_{0}\right)$ конгруэнтных точек.

ЛЕмма 3.4. Пусть $\Omega-C$-плотный ориентируемый растягивающийся аттрактор коразмерности один $u \bar{\Omega}=\pi^{-1}(\Omega)$. Предположим, что имеются точки $\bar{x}, \bar{z} \in \bar{\Omega}$ такие, что $\bar{z} \in w^{s}(\bar{x}) \cap \bar{\Omega}$. Тогда $w^{s *}(\bar{y}) \cap w^{u}(\bar{z}) \neq \varnothing$ для любой точки $\bar{y} \in w^{u}(\bar{x})$. 
ДокАЗАТЕЛЬСтво. Положим

$$
\mathscr{B} \subset w^{u}(\bar{x})=\left\{\bar{b} \in w^{u}(\bar{x}) \mid w^{s *}(\bar{b}) \cap w^{u}(\bar{z}) \neq \varnothing\right\}
$$

Ясно, что $\mathscr{B} \neq \varnothing$, поскольку $\bar{x} \in \mathscr{B}$. В силу теоремы о непрерывной зависимости инвариантных многообразий от начальных условий множество $\mathscr{B}$ открыто в $w^{u}(\bar{x})$. Докажем, что $\mathscr{B}$ замкнуто в $w^{u}(\bar{x})$. Возьмем последовательность точек $\bar{a}_{i} \in \mathscr{B}$ такую, что $\bar{a}_{i} \rightarrow \bar{b}_{0}$ при $i \rightarrow \infty$. В силу леммы 3.2 пересечение $w^{s *}\left(\bar{a}_{i}\right) \cap w^{u}(\bar{z})$ состоит из одной точки, которую обозначим через $\bar{c}_{i}$. Обозначим через $w^{u+}(\bar{x})$ компоненту множества $\bar{M}-w^{u}(\bar{x})$, которая содержит точку $\bar{z}$. В силу леммы 3.1 $[\bar{x}, \bar{z}]^{s} \subset w^{u+}(\bar{x})$ и, следовательно, все точки $\bar{c}_{i}$ принадлежат $w^{u+}(\bar{x})$. Положим $w^{s+}\left(\bar{b}_{0}\right)=w^{s *}\left(\bar{b}_{0}\right) \cap w^{u+}(\bar{x})$.

Предположим сперва, что $(\bar{x}, \bar{z})^{s} \cap \bar{\Omega}=\varnothing$. Тогда $(\bar{x}, \bar{z})^{s}=(\bar{x}, \bar{z})_{\varnothing}^{s}$ и инвариантные многообразия $w^{u}(\bar{x}), w^{u}(\bar{z})$ образуют 2-связку. В силу леммы 2.9 $\bar{\varphi}_{\bar{p} \bar{q}}\left(\bar{b}_{0}\right) \subset w^{u}(\bar{z})$, где $\bar{p} \in w^{u}(\bar{x}), \bar{q} \in w^{u}(\bar{z})$ - ассоциированные точки 2-связки $w^{u}(\bar{x}) \cup w^{u}(\bar{z})$. Поэтому $w^{s *}\left(\bar{b}_{0}\right) \cap w^{u}(\bar{z}) \neq \varnothing$ и $\bar{b}_{0} \in \mathscr{B}$.

Предположим теперь, что $(\bar{x}, \bar{z})^{s} \cap \bar{\Omega} \neq \varnothing$. Согласно лемме 2.9 и предложению 1.5 можно считать, что $\bar{z}$ не является изолированной точкой в $(\bar{x}, \bar{z}]^{s} \cap \bar{\Omega}$. Поэтому существует монотонная последовательность $\bar{x}_{k} \in(\bar{x}, \bar{z}]^{s} \cap \bar{\Omega}$ такая, что $\bar{x}_{k} \rightarrow \bar{z}$ при $k \rightarrow \infty$.

Покажем, что каждое неустойчивое многообразие $w^{u} \subset \bar{\Omega}$, пересекающее $w^{s+}\left(\bar{b}_{0}\right)$ или $\left[\bar{a}_{i}, \bar{c}_{i}\right]^{s}$, пересекает $[\bar{x}, \bar{z}]^{s}$. Отметим, что если $w^{u} \subset \bar{\Omega}$ пересекает $w^{s+}\left(\bar{b}_{0}\right)$, то $w^{u}$ пересекает $\left[\bar{a}_{i}, \bar{c}_{i}\right]^{s}$ для всех достаточно больших $i$. Поэтому достаточно рассмотреть случай, когда $w^{u}$ пересекает $\left[\bar{a}_{i}, \bar{c}_{i}\right]^{s}$. В силу леммы 3.1 точки

$$
\bar{a}_{i} \in w^{u}(\bar{x}), \quad \bar{c}_{i}=w^{s *}\left(\bar{a}_{i}\right) \cap w^{u}(\bar{z}) \subset w^{u}(\bar{z})
$$

лежат в разных компонентах множества $\bar{M}-w^{u}$. Следовательно, $\bar{x}$ и $\bar{z}$ также лежат в разных компонентах $\bar{M}-w^{u}$. Поэтому $w^{u}$ пересекает $[\bar{x}, \bar{z}]^{s}$. В частности, каждое неустойчивое многообразие, содержащее граничную точку и пересекающее $w^{s+}\left(\bar{b}_{0}\right)$ или $\left[\bar{a}_{i}, \bar{c}_{i}\right]^{s}$, пересекает $[\bar{x}, \bar{z}]^{s}$ (см. рис. 8$)$.

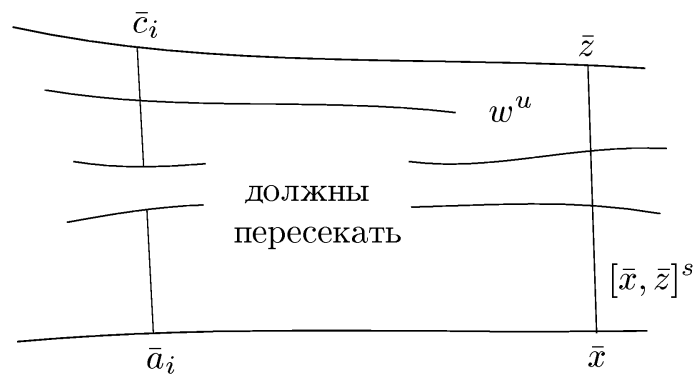

Рис. 8. Пересечение неустойчивых многообразий с дугой $[\bar{x}, \bar{z}]^{s}$ 
Покажем теперь, что каждое неустойчивое многообразие $w^{u} \subset \bar{\Omega}$, пересекающее $[\bar{x}, \bar{z}]^{s}$, пересекает $w^{s+}\left(\bar{b}_{0}\right)$ и, следовательно, $\left[\bar{a}_{i}, \bar{c}_{i}\right]^{s}$. Так как точки $\bar{x}, \bar{z}$ в силу леммы 3.1 лежат в разных компонентах множества $\bar{M}-w^{u}$, то точки

$$
\bar{a}_{i} \in w^{u}(\bar{x}), \quad \bar{c}_{i}=w^{s *}\left(\bar{a}_{i}\right) \cap w^{u}(\bar{z}) \subset w^{u}(\bar{z})
$$

также лежат в разных компонентах множества $\bar{M}-w^{u}$. Предположим, что $w^{u} \cap$ $\left[\bar{a}_{i}, \bar{c}_{i}\right]^{s}=\varnothing$. Тогда $\left[\bar{a}_{i}, \bar{c}_{i}\right]^{s}$ содержит ассоциированные точки, скажем $\bar{p}_{i}$ и $\bar{q}_{i}$. Будем для определенности считать, что $\bar{a}_{i}$ и $\bar{p}_{i}$ (соответственно, $\bar{c}_{i}$ и $\bar{q}_{i}$ ) принадлежат одному устойчивому многообразию.

Вышеприведенные рассуждения показывают, что $w^{u}\left(\bar{p}_{i}\right)$ и $w^{u}\left(\bar{q}_{i}\right)$ пересекают $[\bar{x}, \bar{z}]^{s}$. Неустойчивое многообразие $w^{u}$ не может пересекать ни дугу $\left[\bar{x}, w^{u}\left(\bar{p}_{i}\right) \cap\right.$ $\left.w^{s}(\bar{x})\right]^{s}$, ни дугу $\left[w^{u}\left(\bar{q}_{i}\right) \cap w^{s}(\bar{z}), \bar{z}\right]^{s}$. В противном случае $w^{u}$ должно пересекать $\left[\bar{a}_{i}, \bar{p}_{i}\right]^{s}$ или $\left[\bar{q}_{i}, \bar{c}_{i}\right]^{s}$ соответственно, что противоречит нашему предположению. Поэтому $w^{u}$ пересекает дугу

$$
\left(w^{u}\left(\bar{p}_{i}\right) \cap w^{s}(\bar{x}), w^{u}\left(\bar{q}_{i}\right) \cap w^{s}(\bar{z})\right)^{s}=\left(w^{u}\left(\bar{p}_{i}\right) \cap w^{s}(\bar{x}), w^{u}\left(\bar{q}_{i}\right) \cap w^{s}(\bar{z})\right)_{\varnothing}^{s},
$$

что противоречит лемме 2.9 .

Таким образом, $w^{s+}\left(\bar{b}_{0}\right) \cap w^{u}\left(\bar{x}_{k}\right) \neq \varnothing$. Согласно лемме 3.2 это пересечение состоит из одной точки. Положим $\bar{y}_{k}=w^{s+}\left(\bar{b}_{0}\right) \cap w^{u}\left(\bar{x}_{k}\right)$. В силу леммы 3.3 последовательность точек $\bar{y}_{k}$ ограничена на $w^{s+}\left(\bar{b}_{0}\right)$. Согласно лемме 3.1 последовательность $\bar{y}_{k}$ монотонная, поскольку последовательность $\bar{x}_{k}$ монотонная. Так как $\bar{\Omega}$ компактно, то $\bar{y}_{k} \rightarrow \bar{y}_{*} \in \bar{\Omega}$.

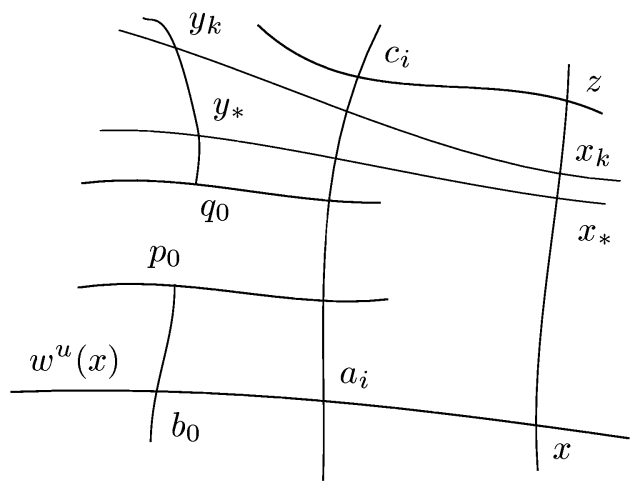

Рис. 9. Последовательность $\bar{y}_{k}$

Покажем, что $w^{u}\left(\bar{y}_{*}\right)=w^{u}(\bar{z})$. Предположим противное. Тогда $w^{u}\left(\bar{y}_{*}\right)$ пересекает $(\bar{x}, \bar{z})^{s}$ в некоторой точке $\bar{x}_{*}$. Так как $\bar{x}_{k} \rightarrow \bar{z}$, то имеется бесконечно много точек $\bar{x}_{k} \in\left(\bar{x}_{*}, \bar{z}\right)^{s}$. Это противоречит тому, что $\bar{y}_{k}$ стремится монотонно к $\bar{y}_{*}$ (см. рис. 9). Таким образом, $w^{u}\left(\bar{y}_{*}\right)=w^{u}(\bar{z})$. Поэтому множество $\mathscr{B}$ замкнуто. Из связности $w^{u}(\bar{x})$ вытекает, что $w^{u}(\bar{x})=\mathscr{B}$.

СЛЕДСТВИЕ 3.1. Пусть $\Omega-C$-плотный ориентируемый растягивающийся аттрактор коразмерности один $u \bar{\Omega}=\pi^{-1}(\Omega)$. Предположим, что $w^{s *}(\bar{x})$ $u w^{s *}(\bar{y})$ пересекают некоторый слой ламинации $\mathscr{L}(\bar{\Omega})$, где $\bar{x}, \bar{y} \in \bar{\Omega}$. Тогда $w^{s *}(\bar{x})$ и $w^{s *}(\bar{y})$ пересекают одни и те же слои ламинации $\mathscr{L}(\bar{\Omega})$. 
ДоКАЗАТЕЛЬСТВО. Согласно лемме 2.9 и теореме о непрерывной зависимости слоев от начальных условий существует точка $\bar{z} \in w^{u}(\bar{x})$ такая, что $\pi\left(w^{s}(\bar{z})\right)$ не содержит граничных периодических точек, и $w^{s}(\bar{z}) \cap w^{u}(\bar{y}) \neq \varnothing$. Отсюда и из леммы 3.4 вытекает требуемый результат.

ЛЕмма 3.5. Пусть $\Omega$ - ориентируемый растягивающийся аттрактор коразмерности один. Предположим, что каждая характеристическая сфера $S_{p_{i} q_{i}}$ на $\Omega$ ограничивает замкнутый шар $E_{p_{i} q_{i}} \subset M$. Пусть $\bar{B}_{\bar{p} \bar{q}}=w^{u}(\bar{p}) \cup$ $w^{u}(\bar{q})-2$-связка множества $\bar{\Omega}=\pi^{-1}(\Omega)$, где $\bar{p}, \bar{q}$ - ассоциированнье точки из $\pi^{-1}(\Omega)$. Пусть $\mathscr{J}_{\bar{p} \bar{q}} \subset \bar{M}$ - открытая область, ограниченная неустойчивыми многообразиями $w^{u}(\bar{p})$ и $w^{u}(\bar{q})$. Тогда $\mathscr{J}_{\bar{p} \bar{q}} \cap \bar{\Omega}=\varnothing$, т.е. область $\mathscr{J}_{\bar{p}} \bar{q}$ не содержит точек из $\bar{\Omega}$.

ДокАЗАТЕЛЬСТво. Согласно следствию $2.5 \pi\left(\bar{B}_{\bar{p} q}\right)=B_{p q}=W^{u}(p) \cup W^{u}(q)$ есть 2-связка множества $\Omega$, где $p=\pi(\bar{p}), q=\pi(\bar{q})$ - периодические ассоциированные точки. Пусть $S_{p q}$-характеристическая сфера, соответствуюшая $B_{p q}$. В силу леммы 2.10 существует характеристическая сфера $\bar{S}_{\bar{p} \bar{q}}$, соответствуюшая $\bar{B}_{\bar{p} \bar{q}}$ и накрывающая $S_{p q}$. Пусть $m$ - общий период точек $p, q$. По условию $S_{p q}$ ограничивает замкнутый шар $E_{p q} \subset M$. Так как шар $E_{p q}$ является односвязным множеством, то из теоремы о накрывающей гомотопии вытекает, что прообраз $\pi^{-1}\left(E_{p q}\right)$ представляет собой объединение замкнутых шаров. Из следствия 2.8 следует, что эти шары попарно не пересекаются, поскольку один шар не может лежать внутри другого. По аналогичной причине прообраз $\pi^{-1}\left(f^{m j}\left(E_{p q}\right)\right)$ представляет собой объединение попарно не пересекающихся замкнутых шаров. Обозначим через $\bar{E}_{\bar{p} q}^{j}$ шар из $\pi^{-1}\left(f^{m j}\left(E_{p q}\right)\right)$, содержащий точки $\bar{p}, \bar{q}$. Положим

$$
\mathscr{J}_{\bar{p} \bar{q}}^{\prime}=\bigcup_{j \geqslant 0} \bar{E}_{\bar{p} \bar{q}}^{j} .
$$

В силу леммы 3.1 множество

$$
\operatorname{clos} \mathscr{J}_{\bar{p} \bar{q}}=\mathscr{J}_{\bar{p} \bar{q}} \cup w^{u}(\bar{p}) \cup w^{u}(\bar{q})
$$

связно, поскольку $\mathscr{J}_{\bar{p} \bar{q}}$ связно и $w^{u}(\bar{p}), w^{u}(\bar{q})$ вложены в $\bar{M}$. Очевидно, $\mathscr{J}_{\bar{p}}^{\prime} \subset$ $\operatorname{clos} \mathscr{J}_{\bar{p} \bar{q}}$. Из теоремы о непрерывной зависимости инвариантных многообразий от начальных условий вытекает, что $\mathscr{J}_{\bar{p} \bar{q}}^{\prime}$ открыто в $\operatorname{clos} \mathscr{J}_{\bar{p} \bar{q}}$. Покажем, что $\mathscr{J}_{\bar{p}}^{\prime}$ замкнуто в $\cos \mathscr{J}_{\bar{p} \bar{q}}$. Возьмем последовательность точек $\bar{a}_{i} \in \operatorname{clos} \mathscr{J}_{\bar{p} \bar{q}}$ такую, что $\bar{a}_{i} \rightarrow \bar{a}_{*}$ при $i \rightarrow \infty$. Тогда $\bar{a}_{i} \in \bar{E}_{\bar{p} \bar{q}}^{j(i)}$ для некоторого $j(i) \geqslant 0$. Если последовательность индексов $j(i)$ ограничена, то $\bar{a}_{*} \in \bar{E}_{\bar{p} \bar{q}}^{j_{*}}$ при некотором $j_{*}$, поскольку $\bigcup_{j \geqslant 0} \bar{E}_{\bar{p} \bar{q}}^{j}$ есть объединение непересекаюшихся шаров. Если последовательность индексов $j(i)$ неограничена, то в силу теоремы $2.1 \bar{a}_{*} \in \bar{\Omega}$. В обоих случаях $\bar{a}_{*} \in \mathscr{J}_{\bar{p} \bar{q}}^{\prime}$. Поэтому $\mathscr{J}_{\bar{p} \bar{q}}^{\prime}$ замкнуто и, следовательно, $\operatorname{clos} \mathscr{J}_{\bar{p} \bar{q}}=\mathscr{J}_{\bar{p} \bar{q}}^{\prime}$. Так как в силу теоремы 2.1 и следствия 2.8 внутри $\bar{E}_{\bar{p} \bar{q}}^{j}$ не могут лежать точки из $\bar{\Omega}$, то в области $\mathscr{J}_{\bar{p} \bar{q}}$ нет неустойчивых многообразий из $\bar{\Omega}$.

ЛЕмма 3.6. Предположим, что каждая характеристическая сфера $S_{p_{i} q_{i}}$ ориентируемого растягивающегося аттрактора коразмерности один $\Omega$ ограничивает замкнутый иар $E_{p_{i} q_{i}} \subset M$. Если устойчивое многообразие $W^{s}(x)$ точки $x \in \Omega$ не содержит граничных периодических точек, то любое его поднятие пересекает все слои ламинации $\mathscr{L}(\bar{\Omega})$. 
ДокАЗАТЕЛЬСТво. В силу теоремы $2.1 \Omega$ является $C$-плотным базисным множеством. Поэтому $W^{s}(x) \cap \Omega$ плотно в $\Omega$. Согласно предложению 1.3 множества $W^{s+}(x) \cap \Omega, W^{s-}(x) \cap \Omega$ плотны в $\Omega$, где $W^{s \pm}(x)$ - компоненты множества $W^{s}(x)-x$. Отсюда следует, что имеется последовательность $\left\{\bar{x}_{i} \in w^{s}(\bar{x}) \cap \bar{\Omega}\right\}_{-\infty}^{+\infty}$ такая, что

$$
\bigcup_{i=-\infty}^{+\infty}\left[\bar{x}_{-i}, \bar{x}_{i}\right]^{s}=w^{s}(\bar{x}), \quad \cdots \subset\left[\bar{x}_{-i}, \bar{x}_{i}\right]^{s} \subset\left[\bar{x}_{-i-1}, \bar{x}_{i+1}\right]^{s} \subset \cdots .
$$

Обозначим через $\mathscr{J}_{i} \subset \bar{M}$ замкнутую область, ограниченную неустойчивыми многообразиями $w^{u}\left(\bar{x}_{i}\right)$ и $w^{u}\left(\bar{x}_{-i}\right)$. В силу леммы 3.1 области $\mathscr{J}_{i}$ образуют последовательность увеличивающихся связных множеств

$$
\mathscr{J}_{1} \subset \mathscr{J}_{2} \subset \cdots, \quad \mathscr{J}_{i} \subset \mathscr{J}_{i+1} \subset \cdots,
$$

где все включения собственные. Множество $\bigcup_{i \in \mathbb{Z}} \mathscr{J}_{i}$ открыто в $\bar{M}$, поскольку каждое $\mathscr{J}_{i}$ является топологическим подмногообразием. Покажем, что $\bigcup_{i \in \mathbb{Z}} \mathscr{J}_{i}$ замкнуто. Возьмем сходящуюся последовательность $\bar{y}_{i} \rightarrow \bar{z}$ при $i \rightarrow \infty$, где $\bar{y}_{i} \in$ $w^{u}\left(\bar{x}_{i}\right)$. Тогда $\bar{z} \in \bar{\Omega}$, поскольку $\bar{\Omega}$ замкнуто. Следовательно, $w^{s *}(\bar{z})$ пересекает $w^{u}\left(\bar{x}_{i}\right)$ для всех достаточно больших $i$. В силу следствия $3.1 w^{u}(\bar{z})$ пересекает $w^{s}(\bar{x})$. Так как последовательность $\bar{x}_{i}$ неограничена на $w^{s}(\bar{x})$, то $w^{u}(\bar{z}) \subset \mathscr{J}_{j}$ для некоторого $j \in \mathbb{N}$, и поэтому $\bar{z} \in \bigcup_{i \in \mathbb{Z}} \mathscr{J}_{i}$. Таким образом, $\bigcup_{i \in \mathbb{Z}} \mathscr{J}_{i}$ замкнуто. Следовательно, $\bigcup_{i \in \mathbb{Z}} \mathscr{J}_{i}=\bar{M}$.

Осталось показать, что каждое неустойчивое многообразие $w^{u} \subset \bar{\Omega}$, лежащее в $w^{u} \subset \mathscr{J}_{i}$ для некоторого $i \in \mathbb{N}$, пересекает $w^{s}(\bar{x})$. Согласно лемме $3.1 \mathscr{J}_{i}$ линейно связно. Возьмем произвольный путь $P \subset \mathscr{J}_{i}$ от точки $\bar{a} \in w^{u}\left(\bar{x}_{i}\right)$ к точке $\bar{b} \in w^{u}$. Положим

$$
P_{a}=\left\{\bar{z} \in P \cap \bar{\Omega} \mid w^{u}(\bar{z}) \cap w^{s}(\bar{x})\right\} \neq \varnothing .
$$

Из предыдущих рассуждений и теоремы о непрерывной зависимости инвариантных многообразий от начальных условий вытекает, что $P_{a}$ замкнуто в $P$. Поэтому сушествует точка $\bar{a}_{*} \in P_{a}$ такая, что $P_{a}$ принадлежит замкнутому промежутку $\left[\bar{a}, \bar{a}_{*}\right] \subset P$ и $\left(\bar{a}_{*}, \bar{b}\right) \cap P_{a}=\varnothing$. Не уменьшая общности, можно считать, что $P$ трансверсально $w^{u}\left(\bar{a}_{*}\right)$ в некоторой точке $\bar{a}_{*}$. Тогда, вновь применяя теорему о непрерывной зависимости инвариантных многообразий от начальных условий, получаем, что $\bar{\Omega}$ не пересекается с $\left(\bar{a}_{*}, \bar{b}\right)$ вблизи $\bar{a}_{*}$. Следовательно, $w^{u}\left(\bar{a}_{*}\right)$ содержит поднятие граничной периодической точки. Обозначим это поднятие через $\bar{p}$ (см. рис. 10).

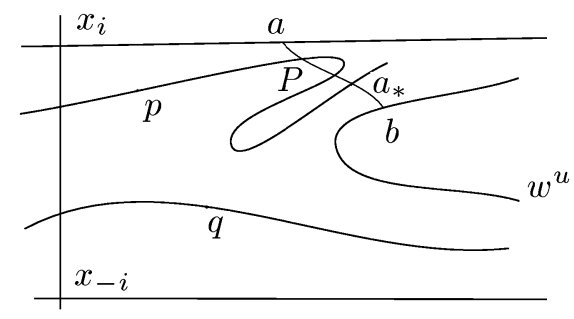

Рис. 10. Ассоциированные точки $\bar{p}$ и $\bar{q}$ 
Пусть $\bar{q}$ - точка, ассоциированная с $\bar{p}$. Обозначим через $\mathscr{J}_{\bar{p} \bar{q}}$ замкнутую область, ограниченную $w^{u}(\bar{p})$ и $w^{u}(\bar{q})$. В силу следствия $3.1 w^{u}(\bar{q}) \cap w^{s}(\bar{x}) \neq \varnothing$. Из определения точки $\bar{a}_{*}$ вытекает, что $w^{u}(\bar{q}) \cap\left(\bar{a}_{*}, \bar{b}\right)=\varnothing$. Так как $\bar{\Omega}$ не пересекает $\left(\bar{a}_{*}, \bar{b}\right)$ вблизи $\bar{a}_{*}$, то $\left(\bar{a}_{*}, \bar{b}\right) \subset \mathscr{J}_{\bar{p} \bar{q}}$. Следовательно, $w^{u} \subset \mathscr{J}_{\bar{p} \bar{q}}$. С другой стороны, согласно лемме 3.5 в $\mathscr{J}_{\bar{p}} \bar{q}$ имеются точки из $\bar{\Omega}$. Полученное противоречие завершает доказательство.

СлЕДСТвИЕ 3.2. Пусть выполнены условия леммы 3.6. Тогда $w^{s *}(\bar{z})$ пересекает все неустойчивые многообразия из множества $\bar{\Omega}, \bar{z} \in \bar{\Omega}$.

ЛЕмма 3.7. Пусть каждая характеристическая сфера $S_{p_{i} q_{i}}$ ориентируемого растягивающегося аттрактора коразмерности один $\Omega$ ограничивает замкнутый шар $E_{p_{i} q_{i}} \subset M$. Тогда универсальная накрывающая $\bar{M}$ гомеоморфна $\mathbb{R}^{n}$.

ДокАЗАТЕЛЬСТво. Возьмем точку $\bar{x}_{0} \in \bar{\Omega}$ такую, что $w^{s}\left(\bar{x}_{0}\right)$ не содержит прообразов граничных периодических точек. Введем на $w^{s}\left(\bar{x}_{0}\right)$ ориентацию и обозначим через

$$
\ldots, \bar{E}_{-j}, \ldots, \bar{E}_{-1}, \bar{E}_{1}, \ldots, \bar{E}_{j}, \ldots
$$

шары из $\pi^{-1}\left(\bigcup_{i=1}^{k} E_{p_{i}} q_{i}\right)$, последовательно пересекаемые кривой $w^{s}\left(\bar{x}_{0}\right)$. В качестве $\bar{E}_{1}$ берем произвольный шар из $\pi^{-1}\left(\bigcup_{i=1}^{k} E_{p_{i} q_{i}}\right)$, пересекаемый кривой $w^{s}\left(\bar{x}_{0}\right)$. В силу леммы 2.9 существует точка входа (соответственно, точка выхода кривой $\left.w^{s}\left(\bar{x}_{0}\right)\right)$ в (из) шар (а) $\bar{E}_{j}$, которую обозначим через $x_{j}^{-}$(соответственно, $x_{j}^{+}$). Не уменьшая обшности, можно считать, что $\bar{x}_{0}$ лежит между $x_{-1}^{+}$и $x_{1}^{-}$.

Обозначим через $\mathbb{R}_{n}^{1}=\left\{\left(0, \ldots, 0, x_{n}\right) \mid x_{n} \in \mathbb{R}\right\}$ ось $x_{n}$ евклидова пространства $\mathbb{R}^{n}$ с декартовыми координатами $\left(x_{1}, \ldots, x_{n-1}, x_{n}\right)$. Эта ось перпендикулярна гиперплоскости $\mathbb{R}^{n-1} \subset \mathbb{R}^{n-1} \times \mathbb{R}_{n}^{1} \cong \mathbb{R}^{n}$, вложенной в $\mathbb{R}^{n}$, которая задается как множество точек с координатой $x_{n}$, равной 0 . Возьмем произвольный сохраняющий ориентацию гомеоморфизм $h: w^{s}\left(\bar{x}_{0}\right) \rightarrow \mathbb{R}_{n}^{1}$ такой, что

$$
\begin{aligned}
h\left(\left[x_{-1}^{+}, x_{1}^{-}\right]^{s}\right) & =[-1,+1], \quad h\left(\bar{x}_{0}\right)=0, \\
h\left(\left[x_{j}^{-}, x_{j}^{+}\right]^{s}\right) & =[j, j+0.5] \quad \text { для } j \geqslant 1, \\
h\left(\left[x_{j}^{-}, x_{j}^{+}\right]^{s}\right) & =[j-0.5, j] \quad \text { для } j \leqslant-1 .
\end{aligned}
$$

Неустойчивое многообразие $w^{u}\left(\bar{x}_{0}\right)$ снабдим некоторой ориентацией и отождествим $w^{u}\left(\bar{x}_{0}\right)$ с гиперплоскостью $\mathbb{R}^{n-1}$ с помощью произвольного гомеоморфизма

$$
V_{0}: w^{u}\left(\bar{x}_{0}\right) \rightarrow \mathbb{R}^{n-1} \subset \mathbb{R}^{n-1} \times \mathbb{R} \cong \mathbb{R}^{n}
$$

такого, что $V_{0}\left(\bar{x}_{0}\right)=0$. Продолжим сперва $V_{0}$ на множество $\bar{\Omega}$. Для этого определим две “проекции" $P_{1}: \bar{\Omega} \rightarrow w^{u}\left(\bar{x}_{0}\right)$ и $P_{2}: \bar{\Omega} \rightarrow w^{s}\left(\bar{x}_{0}\right)$ следуюшим образом. Напомним, что для точки $\bar{b} \in \bar{\Omega}$ полагаем $w^{s *}(\bar{b})=w^{s}(\bar{b})$, если $w^{s}(\bar{b})$ не содержит поднятий граничных периодических точек множества $\Omega$, и $w^{s *}(\bar{b})=w^{s}(\bar{p}) \cup w^{s}(\bar{q})$, если $w^{s}(\bar{b})$ содержит поднятия $\bar{p}, \bar{q}$ граничных периодических точек, при этом $\bar{p}, \bar{q}$ являются ассоциированными точками. В силу леммы 3.1 и следствия 3.2 пересечение $w^{s *}(\bar{b}) \cap w^{u}\left(\bar{x}_{0}\right)$ состоит из одной точки. Аналогично, пересечение $w^{u}(\bar{b}) \cap w^{s}\left(\bar{x}_{0}\right)$ также состоит из одной точки. Положим

$$
\begin{gathered}
P_{1}(\bar{b})=w^{s *}(\bar{b}) \cap w^{u}\left(\bar{x}_{0}\right), \quad P_{2}(\bar{b})=w^{u}(\bar{b}) \cap w^{s}\left(\bar{x}_{0}\right), \quad \bar{b} \in w^{u}(\bar{p}), \\
V(\bar{b})=\left(V_{0}\left(P_{1}(\bar{b})\right), h\left(P_{2}(\bar{b})\right)\right)=\left(V_{0}\left(w^{s *}(\bar{b}) \cap w^{u}\left(\bar{x}_{0}\right)\right), h\left(w^{u}(\bar{b}) \cap w^{s}\left(\bar{x}_{0}\right)\right)\right) .
\end{gathered}
$$


Так как $\bar{\Omega} \cap \operatorname{int} \bar{E}_{j}=\varnothing$ для $j \in \mathbb{Z}-0$, то отображение $V: \bar{\Omega} \rightarrow \mathbb{R}^{n}$ корректно определено и является продолжением отображения $V_{0}$. Для простоты изложения последующие продолжения отображения $V$ будем обозначать той же буквой.

Определим теперь $V$ в точке

$$
\bar{b} \in \bar{M}-\left(\bar{\Omega} \cup \pi^{-1}\left(\bigcup_{i=1}^{k} \operatorname{int} E_{p_{i} q_{i}}\right)\right) .
$$

Так как $\bar{b} \notin \pi^{-1}\left(\bigcup_{i=1}^{k} \operatorname{int} E_{p_{i} q_{i}}\right)$, то в силу теоремы $2.1 \bar{b} \in W^{s}(\bar{\Omega})$. Тогда $\bar{b} \in$ $(\bar{x}, \bar{y})_{\varnothing}^{s}$, где $\bar{x} \in w^{u}(\bar{p}), \bar{y} \in w^{u}(\bar{q})$, и $\bar{p}, \bar{q}-$ ассоциированные точки. Напомним, что $\bar{\varphi}_{\bar{p} \bar{q}}(\bar{y})=\bar{x}$ и $\bar{\varphi}_{\bar{p} \bar{q}}(\bar{x})=\bar{y}$, где гомеоморфизм $\bar{\varphi}_{\bar{p} \bar{q}}(\bar{y})$ удовлетворяет лемме 2.9 . Обозначим через

$$
\lambda^{\prime}(\bar{b})=\frac{\lambda_{\bar{x}}(\bar{b})}{\lambda_{\bar{x}}(\bar{y})}=\frac{\lambda_{\bar{x}}(\bar{b})}{\lambda_{\bar{x}}\left(\bar{\varphi}_{\bar{p} \bar{q}}(\bar{x})\right)}
$$

"относительное" расстояние между точками $\bar{x}, \bar{b}$, где $\lambda_{\bar{x}}(\bar{z})$ - длина отрезка $(\bar{x}, \bar{z})^{s}$. Согласно лемме 3.6 оба пересечения

$$
w^{s}\left(\bar{x}_{0}\right) \cap w^{u}(\bar{x})=P_{2}(\bar{x}), \quad w^{s}\left(\bar{x}_{0}\right) \cap w^{u}(\bar{y})=P_{2}(\bar{y})
$$

не пусты. В силу следствия 3.2 пересечение $w^{s *}(\bar{b}) \cap w^{u}\left(\bar{x}_{0}\right)$ состоит из одной точки. Положим

$$
V(\bar{b})=\left(V_{0}\left(w^{s *}(\bar{b}) \cap w^{u}\left(\bar{x}_{0}\right)\right),\left[1-\lambda^{\prime}(\bar{b})\right] h\left(P_{2}(\bar{x})\right)+\lambda^{\prime}(\bar{b}) h\left(P_{2}(\bar{y})\right)\right) .
$$

Так как $P_{2}(\bar{x}), P_{2}(\bar{y}) \in w^{s}\left(\bar{x}_{0}\right)$, то точки $h\left(P_{2}(\bar{x})\right), h\left(P_{2}(\bar{y})\right)$ определены и, следовательно, $V(\bar{b})$ корректно определено. Нетрудно видеть, что так определенное отображение является продолжением построенного вьше отображения $V: \bar{\Omega} \rightarrow \mathbb{R}^{n}$.

Осталось продолжить $V$ на внутренность шаров $\pi^{-1}\left(\bigcup_{i=1}^{k} E_{p_{i} q_{i}}\right)$. Пусть характеристическая сфера $S_{\bar{p} \bar{q}}=D_{\bar{p}} \cup D_{\bar{q}} \cup C_{\bar{p}} \bar{q}$ ограничивает замкнутый шар $E_{\bar{p} \bar{q}} \subset \pi^{-1}\left(\bigcup_{i=1}^{k} E_{p_{i} q_{i}}\right)$. Покажем, что $V\left(S_{\bar{p} \bar{q}}\right)$ является $(n-1)$-мерной сферой в $\mathbb{R}^{n}$.

В силу леммы 2.10 и теоремы о непрерывной зависимости инвариантных многообразий от начальных условий, $P_{1}\left(\bar{D}_{\bar{p}}\right)$ и $P_{2}\left(\bar{D}_{\bar{q}}\right)$ являются $(n-1)$-мерньми шарами в $w^{u}\left(\bar{x}_{0}\right)$. Более того, $P_{1}\left(\bar{D}_{\bar{p}}\right)=P_{1}\left(\bar{D}_{\bar{q}}\right)$, поскольку $w^{s *}(\bar{x})=w^{s *}\left(\bar{\varphi}_{\bar{p} \bar{q}}(\bar{x})\right)$ для любой точки $\bar{x} \in \bar{D}_{\bar{p}}$ и, следовательно, $P_{1}(\bar{x})=P_{1}\left(\bar{\varphi}_{\bar{p} \bar{q}}(\bar{x})\right)$. Поэтому $V\left(\bar{D}_{\bar{p}}\right)$ и $V\left(\bar{D}_{\bar{q}}\right)$ являются $(n-1)$-мерными шарами в $\mathbb{R}^{n}$, которые получаются один из другого с помошью сдвига

$$
s: \mathbb{R}^{n} \rightarrow \mathbb{R}^{n}, \quad s\left(x_{1}, \ldots, x_{n-1}, x_{n}\right)=\left(x_{1}, \ldots, x_{n-1}, x_{n}+\alpha\right),
$$

вдоль оси $O x_{n}$, где $\alpha=\left|h\left(P_{2}(\bar{x})\right)-h\left(P_{2}(\bar{y})\right)\right|, \bar{y}=\bar{\varphi}_{\bar{p} \bar{q}}(\bar{x})$. Так как по построению любой отрезок $(\bar{x}, \bar{y})^{s}$, где $\bar{x} \in \partial \bar{D}_{\bar{p}}, \bar{y} \in \partial \bar{D}_{\bar{q}}$, отображается под действием $V$ в отрезок

$$
\left[V_{0}\left(P_{1}(\bar{x})\right), t\right], \quad h\left(P_{2}(\bar{x})\right) \leqslant t \leqslant h\left(P_{2}(\bar{x})\right)+\alpha,
$$

длины $\left|h\left(\bar{x}_{\perp}\right)-h\left(\bar{y}_{\perp}\right)\right|$, то объединение

$$
V\left(C_{\bar{p} \bar{q}}\right)=\bigcup_{\bar{x} \in \bar{S}_{\bar{p}}} V\left(\left[\bar{x}, \bar{\varphi}_{\bar{p} \bar{q}}(\bar{x})\right]\right)
$$


образует $(n-1)$-мерный цилиндр в $\mathbb{R}^{n}$. Отсюда вытекает, что $V\left(S_{\bar{p} \bar{q}}\right)$ является $(n-1)$-мерной сферой в $\mathbb{R}^{n}$. В силу теоремы о непрерывной зависимости инвариантных многообразий от начальных условий, $V\left(S_{\bar{p} \bar{q}}\right)$ является цилиндрически вложенной сферой. Поэтому $V\left(S_{\bar{p} \bar{q}}\right)$ ограничивает $n$-мерный шар, скажем $E_{V(\bar{p} \bar{q})}[53]$.

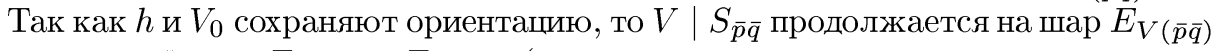
до гомеоморфизма $E_{\bar{p} \bar{q}} \rightarrow E_{V(\bar{p} \bar{q})}$ (такое продолжение существует; см., например, [18]). Таким образом, $V$ гомеоморфно продолжается внутрь всех шаров из $\pi^{-1}\left(\bigcup_{i=1}^{k} E_{p_{i} q_{i}}\right)$. Это завершает построение отображения $V: \bar{M} \rightarrow \mathbb{R}^{n}$, поскольку $\bar{M}=W^{s}(\bar{\Omega}) \cup\left(\pi^{-1}\left(\bigcup_{i=1}^{k} E_{p_{i} q_{i}}\right)\right)$. Отметим, что если $E_{\bar{p} \bar{q}}=\bar{E}_{j}$ для некоторого $j$, то, вообще говоря, $V$ не совпадает с $h$ на $\bar{E}_{j} \cap w^{s}\left(\bar{x}_{0}\right)$.

В силу построения и непрерьвной зависимости инвариантных многообразий от начальных условий, $V$ является взаимно однозначным отображением на свой образ и локальным гомеоморфизмом. Покажем, что $V$ является собственным отображением. Предположим противное. Тогда сушествует последовательность точек $\bar{b}_{j} \in \bar{M}$, не имеющая точек накопления и такая, что последовательность $V\left(\bar{b}_{j}\right)$ ограничена. Обозначим через

$$
\begin{aligned}
\operatorname{pr}_{1}: \mathbb{R}^{n} \rightarrow \mathbb{R}, \quad \operatorname{pr}_{1}\left(x_{1}, \ldots, x_{n-1}, x_{n}\right)=\left(x_{1}, \ldots, x_{n-1}, 0\right) \\
\operatorname{pr}_{n}: \mathbb{R}^{n} \rightarrow \mathbb{R}, \quad \operatorname{pr}_{n}\left(x_{1}, \ldots, x_{n-1}, x_{n}\right)=\left(0, \ldots, 0, x_{n}\right)
\end{aligned}
$$

естественные проекции. Тогда обе последовательности $\operatorname{pr}_{n}\left(V\left(\bar{b}_{j}\right)\right)$ и $\operatorname{pr}_{1}\left(V\left(\bar{b}_{j}\right)\right)$ ограничены. Если $\bar{b}_{j} \in \pi^{-1}\left(\bigcup_{i=1}^{k} E_{p_{i} q_{i}}\right)$, то, не уменьшая общности, можно считать, что $\bar{b}_{j} \in \pi^{-1}\left(\bigcup_{i=1}^{k} S_{p_{i} q_{i}}\right)$, поскольку семейство характеристических сфер $S_{p_{i} q_{i}}$ конечно и, следовательно, $\operatorname{diam} \bar{E}<k_{0}<\infty$ для некоторого $k_{0}>0$ и любого шара $\bar{E} \subset \pi^{-1}\left(\bigcup_{i=1}^{k} E_{p_{i}} q_{i}\right)$. Таким образом, далее можно рассматривать последовательность точек $P_{1}\left(\bar{b}_{j}\right)$, так как $\bar{b}_{j}$ лежат на устойчивых многообразиях точек из $\bar{\Omega}$. Если эта последовательность $P_{1}\left(\bar{b}_{j}\right)$ не имеет точек накопления в $w^{u}\left(\bar{x}_{0}\right)$, то в силу леммы 3.1 последовательность $P_{1}\left(\bar{b}_{j}\right)$ не ограничена в топологии неустойчивого многообразия $w^{u}\left(\bar{x}_{0}\right)$. Тогда последовательность $V\left(\bar{b}_{j}\right)=\left(V_{0}\left(P_{1}\left(\bar{b}_{j}\right)\right), \cdot\right)=\left(\operatorname{pr}_{1}\left(\bar{b}_{j}\right), \cdot\right)$ должна быть неограниченной, поскольку $V_{0}$ - гомеоморфизм. Следовательно, можно считать, что $P_{1}\left(\bar{b}_{j}\right)$ сходится в топологии $w^{u}\left(\bar{x}_{0}\right)$.

Обозначим через $\mathscr{J}_{j}$ область, ограниченную неустойчивыми многообразиями $w^{u}\left(x_{j}^{+}\right)$и $w^{u}\left(x_{-j}^{-}\right)$. Так как

$$
h\left(\left[x_{-j}^{-}, x_{j}^{+}\right]\right)=[-(j+0.5) ; j+0.5]
$$

и последовательность $\operatorname{pr}_{n}\left(V\left(\bar{b}_{j}\right)\right)$ ограничена, то точки $\bar{b}_{j}$ лежат в $\mathscr{J}_{k}$ для некоторого $k \in \mathbb{N}$. Поэтому последовательность $\bar{b}_{j}$ также ограничена. Полученное противоречие показывает, что $V$ является собственным отображением и, следовательно, гомеоморфизмом на свой образ.

Осталось доказать, что $V$ является сюръекцией. Возьмем произвольную точку

$$
v=\left(v_{n-1}, v_{n}\right) \in \mathbb{R}^{n-1} \times \mathbb{R}^{1}=\mathbb{R}^{n} .
$$

Так как $V_{0}$ является гомеоморфизмом, то существует точка $z_{0} \in w^{u}\left(\bar{x}_{0}\right)$ такая, что $V_{0}\left(z_{0}\right)=v_{n-1}$. Согласно следствию $3.2 w^{s *}\left(z_{0}\right)$ пересекает все неустойчивые 
многообразия из $\bar{\Omega}$. Отсюда вытекает, что найдутся точки $z_{1}, z_{2} \in w^{s *}\left(z_{0}\right) \cap \bar{\Omega}$ такие, что $\operatorname{pr}_{n} V\left(z_{1}\right) \leqslant v_{n} \leqslant \operatorname{pr}_{n} V\left(z_{2}\right)$. Рассмотрим сперва случай, когда точки $z_{1}, z_{2} \in w^{s *}\left(z_{0}\right)$ соединяются отрезком устойчивого многообразия. Если отрезок $\left[z_{1}, z_{2}\right]^{s}$ не пересекает шары из $\pi^{-1}\left(\bigcup_{i=1}^{k} E_{p_{i} q_{i}}\right)$, то из построения $V$ следует, что существует точка $z_{3} \in\left[z_{1}, z_{2}\right]^{s} \mathrm{c} \mathrm{pr}_{n} V\left(z_{3}\right)=v_{n}$. Тогда $V\left(z_{3}\right)=v$, и $z_{3}$ есть искомая точка. Предположим теперь, что отрезок $\left[z_{1}, z_{2}\right]^{s}$ пересекает шары из $\pi^{-1}\left(\bigcup_{i=1}^{k} E_{p_{i} q_{i}}\right)$. Из конечности числа шаров, пересекаюших $\left[z_{1}, z_{2}\right]^{s}$, вытекает, что достаточно рассмотреть случай, когда $z_{1}$ есть точка входа в некоторый шар $E_{\bar{p} \bar{q}} \subset \pi^{-1}\left(\bigcup_{i=1}^{k} E_{p_{i} q_{i}}\right)$, а $z_{2}$ - точка выхода. Тогда $v \in V\left(E_{\bar{p} \bar{q}}\right)$, что влечет существование точки $z_{3} \in E_{\bar{p} \bar{q}} \mathrm{c} V\left(z_{3}\right)=v$.

Наконец, если точки $z_{1}, z_{2} \in w^{s *}\left(z_{0}\right)$ не соединяются отрезком устойчивого многообразия, то $w^{s *}\left(z_{0}\right)$ содержит поднятия граничных периодических точек, являюшихся ассоциированными точками, которые лежат "между" $z_{1}, z_{2} \in w^{s *}\left(z_{0}\right)$. Не уменьшая обшности, можно считать, что точки $z_{1}, z_{2}$ являются ассоциированными. Тогда $v \in V\left(E_{\bar{p}}\right)$, и поэтому существует $z_{3} \in V\left(E_{z_{1}} z_{2}\right)$ с $V\left(z_{3}\right)=v$. Это завершает доказательство леммы.

Tеорема 3.1. Пусть $f-A$-диффеоморфизм $n$-мерного замкнутого многообразия $M^{n}, \quad n \geqslant 3$, и пусть $\Omega$ - ориентируемый растягивающийся (сжимающийся) аттрактор (репеллер) коразмерности один диффеоморфизма $f$. Предположим, что каждая характеристическая сфера $S_{p_{i} q_{i}}$ базисного множества $\Omega$ ограничивает $n$-мерный шар $E_{p_{i} q_{i}} \subset M^{n}$. Тогда:

1) любое отличное от $\Omega$ базисное множество диффеоморфизма $f$ лежит в $\bigcup_{i=1}^{k} E_{p_{i} q_{i}}$, где оббединение берется по всем парам $\left\{\left(p_{i}, q_{i}\right)\right\}_{i=1}^{k}$ ассочиированных граничных периодических точек множсества $\Omega$;

2) $\Omega$ - единственный ориентируемый растягивающийся (сжимающийся) аттрактор (репеллер) коразмерности один диффеоморфизма $f$;

3) универсальное накрывающее пространство $\bar{M}^{n}$ гомеоморфно $\mathbb{R}^{n}$;

4) фундаментальная группа $\pi_{1}\left(M^{n}\right)$ изоморфна целочисленной решетке $\mathbb{Z}^{n}$; в частности, $M^{n}$ гомотопически әквивалентно $n$-мерному тору $T^{n}$.

ДокАЗАТЕЛЬСТво. Утверждение 1) следует из теоремы 2.1 , поскольку устойчивые многообразия базисных множеств попарно не пересекаются. Утверждения 2) и 3 ) вытекают из следствия 2.7 и леммы 3.7 соответственно. Доказательство утверждения 4) следует доказательству второй половины теоремы 5.1 из [20], где аналогичньй результат доказьвается для многообразий, допускающих слоения с тривиальной группой голономии (интересующий нас частный случай получается для слоений из плоскостей коразмерности один, к этому случаю можно свести доказательство, используя теорему Плыкина о вложении аттрактора в слоение $[26$, c. 93]). Для удобства читателя мы схематично приведем прямое доказательство. Для краткости обозначений положим $M=M^{n}$.

Возьмем точку $\bar{x} \in \bar{\Omega}$ такую, что $w^{s}(\bar{x})$ не содержит поднятий граничных периодических точек. Согласно леммам 3.1 и $3.6 \bar{w}^{s}(\bar{x})$ пересекает каждоенеустойчивое многообразие $\bar{w}^{u} \subset \bar{\Omega}$ ровно в одной точке. Тогда каждый элемент $\alpha$ фундаментальной группы $\pi_{1}\left(M^{n}\right)$ индуцирует на $\bar{w}^{s}(\bar{x}) \cap \bar{\Omega}$ отображение $\mathscr{R}(\alpha)$ следуюшим образом:

$$
\bar{z} \mapsto w^{s}(\bar{x}) \cap \alpha\left(w^{u}(\bar{z})\right), \quad \bar{z} \in w^{s}(\bar{x}) \cap \bar{\Omega} .
$$

Обозначим через $\mathscr{R}\left(\pi_{1}\left(M^{n}\right)\right)$ группу, образованную отображениями $\mathscr{R}(\alpha)$, где 
$\alpha$ пробегает $\pi_{1}\left(M^{n}\right)$. Очевидно, соответствие $\pi_{1}\left(M^{n}\right) \rightarrow \mathscr{R}\left(\pi_{1}\left(M^{n}\right)\right)$ является гомоморфизмом. Так как любое неустойчивое многообразие из $\bar{\Omega}$ не содержит конгруэнтных точек, то группа $\mathscr{R}\left(\pi_{1}(M)\right)$ состоит из преобразований, которые не имеют неподвижных точек. Следовательно, $\mathscr{R}\left(\pi_{1}(M)\right)$ изоморфна фундаментальной группе $\pi_{1}(M)$.

Введем на $w^{s}(\bar{x})$ ориентацию (т.е. зададим положительное направление). Для произвольной точки $\bar{z}_{0} \in w^{s}(\bar{x}) \cap \bar{\Omega}$ существуют точки $\bar{z}_{1}, \bar{z}_{2} \in w^{s}(\bar{x}) \cap \bar{\Omega}$ такие, что $\bar{z}_{1}<\bar{z}_{0}<\bar{z}_{2}$. В силу леммы $3.1 w^{u}\left(\bar{z}_{0}\right)$ принадлежит области $\mathscr{J}_{12}$, ограниченной $w^{u}\left(\bar{z}_{1}\right)$ и $w^{u}\left(\bar{z}_{2}\right)$. Из лемм $3.1,3.6$ вытекает, что пересечение $\mathscr{R}(\alpha)\left(\mathscr{J}_{12}\right) \cap w^{s}(\bar{x})$ есть интервал. Поэтому точка $\alpha\left(\bar{z}_{0}\right)$ лежит между точками $\alpha\left(\bar{z}_{1}\right), \alpha\left(\bar{z}_{2}\right)$. Следовательно, $\mathscr{R}(\alpha)$ - непрерывное отображение. В силу леммы $3.1 \mathscr{R}(\alpha)$ взаимно однозначно. Отсюда получаем, что $\mathscr{R}(\alpha)$ есть гомеоморфизм $\bar{w}^{s}(\bar{x}) \cap \bar{\Omega} \rightarrow \bar{w}^{s}(\bar{x}) \cap \bar{\Omega}$.

Для произвольного $\mathscr{R}(\alpha) \in \mathscr{R}\left(\pi_{1}\left(M^{n}\right)\right)$ покажем, что если $\mathscr{R}(\alpha)\left(t_{0}\right)>t_{0}$ для некоторой точки $t_{0} \in w^{s}(\bar{x}) \cap \bar{\Omega}$, то $\mathscr{R}(\alpha)(t)>t$ для любой $t \in w^{s}(\bar{x}) \cap \bar{\Omega}$. Предположим противное. Тогда существует точка $t_{0}^{\prime} \in w^{s}(\bar{x}) \cap \bar{\Omega}$ такая, что $\mathscr{R}(\alpha)\left(t_{0}^{\prime}\right)<t_{0}^{\prime}$. Положим

$$
P_{u}=\left\{t \in w^{s}(\bar{x}) \cap \bar{\Omega} \mid \mathscr{R}(\alpha)(t)>t\right\}, \quad P_{l}=\left\{t \in w^{s}(\bar{x}) \cap \bar{\Omega} \mid \mathscr{R}(\alpha)(t)<t\right\} .
$$

Сперва покажем, что оба множества $P_{u}$ и $P_{l}$ замкнуты. Возьмем последовательность $t_{i} \in P_{u}$ такую, что $t_{i} \rightarrow t_{*}$. Тогда $t_{*} \in w^{s}(\bar{x}) \cap \bar{\Omega}$, поскольку множество $w^{s}(\bar{x}) \cap \bar{\Omega}$ замкнуто. Так как $\mathscr{R}(\alpha)$ - непрерьвное отображение, то $\mathscr{R}(\alpha)\left(t_{*}\right) \geqslant t_{*}$ и, следовательно, $\mathscr{R}(\alpha)\left(t_{*}\right)>t_{*}$, поскольку $\mathscr{R}(\alpha)$ не имеет неподвижных точек. Отсюда вытекает, что $P_{u}$ замкнуто. Аналогично доказывается замкнутость множества $P_{l}$.

Для определенности будем считать, что $t_{0}<t_{0}^{\prime}$ (в противном случае можно перейти к $\left.\mathscr{R}\left(\alpha^{-1}\right)\right)$. Так как $P_{u}$ и $P_{l}$ замкнуты, то sup $P_{u} \in P_{u}$ и $\inf P_{l} \in P_{l}$. Более того, $\left(\sup P_{u}, \inf P_{l}\right)^{s} \cap \bar{\Omega}=\varnothing$. В силу предложения 1.3 точка $\sup P_{u}$ принадлежит неустойчивому многообразию $w^{u}(\bar{p})$ с граничной точкой $\bar{p}$. Аналогично, inf $P_{l}$ принадлежит $w^{u}(\bar{q})$ с граничной точкой $\bar{q}$. Согласно лемме 2.9 точки $\bar{p}$ и $\bar{q}$ ассоциированные, поскольку $\left(\sup P_{u}, \inf P_{l}\right)^{s}=\left(\sup P_{u}, \inf P_{l}\right)_{\varnothing}^{s}$.

Обозначим через $\mathscr{J}_{\bar{p} \bar{q}} \subset \bar{M}$ открытую область, ограниченную неустойчивыми многообразиями $w^{u}(\bar{p})$ и $w^{u}(\bar{q})$. Согласно лемме $3.5 \mathscr{J}_{\bar{p}} \cap \bar{\Omega}=\varnothing$ и, следовательно, $\alpha\left(\mathscr{J}_{\bar{p} \bar{q}}\right) \cap \bar{\Omega}=\varnothing$. Поэтому $\mathscr{R}(\alpha)\left(\left(\sup P_{u}, \inf P_{l}\right)^{s}\right)$ есть интервал такой, что $\mathscr{R}(\alpha)\left(\left(\sup P_{u}, \inf P_{l}\right)^{s}\right) \cap \bar{\Omega}=\varnothing$. Отсюда получаем, что если $\mathscr{R}(\alpha)\left(\sup P_{u}\right)>\inf P_{l}$, то $\mathscr{R}(\alpha)\left(\sup P_{l}\right)>\inf P_{l}$. Следовательно,

$$
\mathscr{R}(\alpha)\left(\sup P_{u}\right)=\inf P_{l}, \quad \alpha\left(w^{u}(\bar{p})\right)=w^{u}(\bar{q}) .
$$

Тогда $\pi\left(w^{u}(\bar{p})\right)=\pi\left(w^{u}(\bar{q})\right)$, что означает, что $\Omega$ имеет 1-связку. Это противоречит ориентируемости $\Omega$.

Таким образом, $\mathscr{R}(\alpha)(t)>t$ для всех $t \in w^{s}(\bar{x}) \cap \bar{\Omega}$, если только $\mathscr{R}(\alpha)\left(t_{0}\right)>t_{0}$ для некоторого $t_{0} \in w^{s}(\bar{x}) \cap \bar{\Omega}$. Это утверждение позволяет ввести отношение порядка на группе $\mathscr{R}\left(\pi_{1}(M)\right)$ следуюшим образом:

$$
\mathscr{R}(\alpha)>\mathscr{R}(\beta), \quad \text { если } \mathscr{R}(\alpha)(\bar{z})>\mathscr{R}(\beta)(\bar{z})
$$

для одного (и, следовательно, для всех) $\bar{z} \in w^{s}(\bar{x}) \cap \bar{\Omega}$, где $\alpha, \beta \in \pi_{1}(M)$. Так как каждое неустойчивое многообразие из $\Omega$ в силу теоремы 2.1 всюду плотно в $\Omega$, то 
$\mathscr{R}\left(\pi_{1}(M)\right)$ имеет архимедово упорядочивание: для $\mathscr{R}(\alpha) \neq \mathscr{R}(\beta)$ найдется целое $m$ такое, что

$$
\mathscr{R}(\alpha)^{m}>\mathscr{R}(\beta) \text { или } \mathscr{R}(\alpha)^{-m}>\mathscr{R}(\beta) .
$$

В силу теоремы Гёльдера об архимедово упорядоченных группах группа

$$
\mathscr{R}\left(\pi_{1}(M)\right) \cong \pi_{1}(M)
$$

свободная абелева. Из компактности многообразия $M$ вытекает, что фундаментальная группа $\pi_{1}(M)$ конечно порожденная [49] и, следовательно, изоморфна целочисленной решетке $\mathbb{Z}^{r}$. Из доказанного п. 3) следует, что $\pi_{r}\left(M^{n}\right)=0$ при $r \geqslant 2$, т.е. $M$ является так называемым $K(\pi, 1)$-пространством. Отсюда и из компактности $M$ получаем $k=n$. Таким образом,

$$
\pi_{r}\left(M^{n}\right)=0=\pi_{r}\left(T^{n}\right), \quad r \geqslant 2, \quad \pi_{1}\left(M^{n}\right)=\mathbb{Z}^{n}=\pi_{1}\left(T^{n}\right) .
$$

Это означает, что $M^{n}$ слабо гомотопно тору $T^{n}$. Так как $M^{n}$ является $K(\pi, 1)$-пространством, то $M^{n}$ гомотопически эквивалентно $T^{n}$.

\section{§4. О существовании нетрансверсальных пересечений инвариантных многообразий}

В этом параграфе будем придерживаться обозначений $\S 2$. В частности, $\Omega$ - ориентируемый растягиваюшийся аттрактор коразмерности один $A$-диффеоморфизма $f: M \rightarrow M n$-мерного замкнутого многообразия $M=M^{n}, n \geqslant 3$. В следующей теореме рассматривается характеристическая сфера $S_{p q}$, соответствуюшая 2 -связке $W^{u}(p) \cup W^{u}(q)$ базисного множества $\Omega$, где $p, q \in \Omega$-ассоциированные граничные периодические точки. Напомним, что $S_{p q}$ есть объединение замкнутых $(n-1)$-мерных шаров $D_{p} \subset W^{u}(p), D_{q} \subset W^{u}(q)$ и связываюшего $(n-1)$-мерного цилиндра

$$
C_{p q}=\bigcup_{x \in \partial D_{p}, y \in \partial D_{q}}(x, y)_{\varnothing}^{s}, \quad p \in \operatorname{int} D_{p}, \quad q \in \operatorname{int} D_{q} .
$$

Напомним также, что

$$
D_{p q}=\bigcup_{x \in W^{u}(p)-\operatorname{int} D_{p}}\left[x, \varphi_{p q}(x)\right]_{\varnothing}^{s} .
$$

ТЕОрема 4.1. Пусть $f$ - структурно устойчивый диффеоморфизм замкнутого п-мерного многообразия $M^{n}, n \geqslant 3, u \Omega$ - ориентируемый растягивающийся аттрактор коразмерности один диффеоморфизма $f$. Пусть $\Omega^{\prime} \neq \Omega-$ базисное множество индекса $n-1$ такое, что $W^{u}\left(\Omega^{\prime}\right) \cap D_{p q} \neq \varnothing$, и пусть $C \subset D_{p q} \cap W^{u}\left(z^{\prime}\right)$ - компонента пересечения $D_{p q} \cap W^{u}\left(z^{\prime}\right)$, где $z^{\prime} \in \Omega^{\prime}-$ периодическая точка. Тогда

$$
W^{u}\left(z^{\prime}\right) \cap C_{p q}=C \cap C_{p q} \neq \varnothing,
$$

и это пересечение состоит ровно из одной $(n-2)$-мерной сферы, скажем $S^{n-2}$, которая изотопна обеим сферам $S_{p}=\partial D_{p}, S_{q}=\partial D_{q}$ в иилиндре $C_{p q}$. В частности, $S^{n-2}$ разбивает $C_{p q}$ на два $(n-1)$-мерных чилиндра, каждый из которых гомеоморфен $\Sigma^{n-2} \times[0 ; 1]$. 
ДокАЗАТЕЛЬСТво. Согласно теореме Мане [51] $f$ удовлетворяет сильному условию трансверсальности. В силу следствия 2.4 и того, что $W^{u}\left(\Omega^{\prime}\right) \cap D_{p q} \neq \varnothing$, получаем $W^{u}\left(z^{\prime}\right) \cap C_{p q} \neq \varnothing$ и $C \cap C_{p q} \neq \varnothing$. Согласно сильному условию трансверсальности пересечение $W^{u}\left(z^{\prime}\right) \cap C_{p q}$ локально есть $(n-2)$-мерное подмногообразие, трансверсальное любому отрезку $\left(x, \varphi_{p q}(x)\right)_{\varnothing}^{s}, x \in S_{p}=\partial D_{p}$. Обозначим через $S$ компоненту пересечения $C \cap C_{p q}$. Докажем, что $S$ является $(n-2)$-мерной сферой и $W^{u}\left(z^{\prime}\right) \cap C_{p q}=S$.

Напомним, что согласно лемме 2.7 тройка $\left(D_{p q}, W^{u}(p)-\operatorname{int} D_{p}, \pi_{p}\right)$ есть тривиальное расслоение, слоем которого является промежуток $[0 ; 1]$, где $\pi_{p}: D_{p q} \rightarrow$ $W^{u}(p)-\operatorname{int} D_{p}$ - проекция, переводящая каждую точку $b \in D_{p q}$ в $x \in W^{u}(p)-$ int $D_{p}$, если $b \in\left[x, \varphi_{p q}(x)\right]_{\varnothing}^{s} \subset D_{p q}$.

Для удобства разобьем доказательство теоремы на шаги.

ШАГ 4.1. Для любой точки $x_{0} \in W^{u}(p)-p$ существует окрестность $U_{0}$ этой точки в $W^{u}(p)$ со следующим свойством: если $\pi_{p}^{-1}\left(x_{0}\right)$ пересекает неустойчивое многообразие $W^{u}(\theta)$ точки $\theta$ из некоторого базисного множества $\Theta$ индекса $n-1$, то любая компонента пересечения $\pi_{p}^{-1}\left(U_{0}\right) \cap W^{u}(\theta)$ отображсатся посредством $\pi_{p}$ гомеоморфно на $U_{0}$.

ДокАЗАТЕльство. Как доказал Смейл [66], $M^{n}$ есть объединение попарно не пересекающихся неустойчивых многообразий базисных множеств. Отсюда и из сильного условия трансверсальности вытекает, что каждая точка $x \in \pi_{p}^{-1}\left(x_{0}\right)$ принадлежит либо неустойчивому $n$-мерному многообразию, либо неустойчивому $(n-1)$-мерному многообразию. Обозначим через $D_{n-1}\left(\right.$ соответственно, $\left.D_{n}\right)$ подмножество точек отрезка $\pi_{p}^{-1}\left(x_{0}\right)$, принадлежащих неустойчивым $(n-1)$-мерным (соответственно, $n$-мерным) многообразиям точек базисных множеств, отличных от $\Omega$. Так как неустойчивое $n$-мерное многообразие открыто в $M^{n}$, то $D_{n}$ открыто в $\pi_{p}^{-1}\left(x_{0}\right)$. Следовательно, $D_{n-1}-$ компактное множество. Отсюда вытекает, что углы между дугой $\pi_{p}^{-1}\left(x_{0}\right)$ и неустойчивыми $(n-1)$-мерньми многообразиями, проходящими через $D_{n-1}$, отделены от нуля некоторой положительной константой. Поэтому сушествует окрестность $U_{0}^{\prime}$ точки $x_{0}$ в $W^{u}(p)$ такая, что для любого неустойчивого многообразия $W^{u}$, проходящего через $D_{n-1}$, любая компонента пересечения $W^{u} \cap \pi_{p}^{-1}\left(U_{0}^{\prime}\right)$ пересекает все дуги $\pi_{p}^{-1}(x), x \in U_{0}^{\prime}$. Так как $\pi_{p}$ является локально тривиальным расслоением (даже просто тривиальным, но в данном случае достаточно локальной тривиальности), то, уменьшив, если необходимо, окрестность $U_{0}^{\prime}$, можно добиться того, чтобы компонента пересечения $W^{u} \cap \pi_{p}^{-1}\left(U_{0}^{\prime}\right)$ пересекала каждую дугу $\pi_{p}^{-1}(x), x \in U_{0}^{\prime}$, ровно в одной точке. Отсюда следует требуемый результат, поскольку локально компоненты из $W^{u} \cap \pi_{p}^{-1}\left(U_{0}^{\prime}\right)$ попарно не пересекаются.

Далее, когда будем говорить, что $A \subset W^{u}(p)-\operatorname{int} D_{p}$ открыто в $W^{u}(p)-\operatorname{int} D_{p}$, будем иметь в виду внутреннюю топологию многообразий $W^{u}(p)$ и $W^{u}(p)-\operatorname{int} D_{p}$. Обозначим через $\left.\pi_{p}\right|_{C}: C \rightarrow W^{u}(p)-\operatorname{int} D_{p}$ ограничение $\pi_{p}$ на $C$.

ШАГ 4.2. Множество $\pi_{p}(C)$ открыто в $W^{u}(p)-\operatorname{int} D_{p},\left.u \pi_{p}\right|_{C}$ является накрывающим отображением на свой образ $\pi_{p}(C)$.

ДокАЗАТЕЛЬСТВо. Возьмем произвольную точку $x \in \pi_{p}(C)$. Из сильного условия трансверсальности следует, что пересечение $\pi_{p}^{-1}(x) \cap C$ состоит из не более чем счетного множества точек $x_{i} \in D_{p q}$. Очевидно, каждая $x_{i}$ является внутренней точкой многообразия $W^{u}\left(z^{\prime}\right)$ и, следовательно, внутренней точкой $C$ в 
топологии, индуцируемой множеством $D_{p q}$ в $C \subset W^{u}\left(z^{\prime}\right) \cap D_{p q}$ как на подмножестве. Поэтому $\left.\pi_{p}\right|_{C}\left(x_{i}\right)$ есть внутренняя точка множества $\pi_{p}(C)$, поскольку $\left.\pi_{p}\right|_{C}$ является локальным гомеоморфизмом в $x_{i}$. Следовательно, $\pi_{p}(C)$ открыто в $W^{u}(p)-\operatorname{int} D_{p}$.

Пусть $U(x) \subset W^{u}(p)-\operatorname{int} D_{p}$ - окрестность $x$ в $W^{u}(p)-\operatorname{int} D_{p}$, удовлетворяющая шагу 4.1 , и $U\left(x_{i}\right)$ - компонента из $C \cap \pi_{p}^{-1}(U(x))$, содержащая $x_{i}$. Каждая $U\left(x_{i}\right)$ есть окрестность точки $x_{i}$, так как $\left.\pi_{p}\right|_{C}$ - непрерывное отображение. В силу шага 4.1 окрестности $U\left(x_{i}\right)$ попарно не пересекаются и ограничение $\left.\pi_{p}\right|_{C \cap U\left(x_{i}\right)}$ является гомеоморфизмом на $U(x)$. Это завершает доказательство.

Так как $\pi_{p}$ является тривиальным расслоением, то для любого множества $N \subset$ $W^{u}(p)-\operatorname{int} D_{p}$ ограничение $\left.\pi_{p}\right|_{\pi_{p}^{-1}(N)}$ также является тривиальным расслоением со слоем $[0 ; 1]$. Поэтому ограничение

$$
\left.P \stackrel{\text { def }}{=} \pi_{p}\right|_{\operatorname{clos} C_{p q}}: \operatorname{clos} C_{p q} \rightarrow S_{p}^{n-2}, \quad \operatorname{clos} C_{p q}=C_{p q} \cup S_{p}^{n-2} \cup S_{q}^{n-2}
$$

является тривиальным расслоением со слоем $[0 ; 1]$ и тотальным пространством $\operatorname{clos} C_{p q}=\pi_{p}^{-1}\left(S_{p}^{n-2}\right)$.

Для формулировки следуюшего шага напомним, что символом $S$ мы обозначили компоненту пересечения $C \cap C_{p q}$.

ШАГ 4.3. Отображсение $\left.\pi_{p}\right|_{C}: C \rightarrow W^{u}(p)-$ int $D_{p}$ сюрдективно, т.е. $\pi_{p}(C)=W^{u}(p)-\operatorname{int} D_{p} . \quad B$ частности, $\left.P\right|_{S}: S \rightarrow S_{p}^{n-2}$ есть сюргекиия, $P(S)=S_{p}^{n-2}$.

ДокАЗАТЕЛЬСтво. Предположим противное. Поскольку (см. шаг 4.2) $\left.\partial \pi_{p}\right|_{C}(C) \neq \varnothing$, то сушествует точка $\left.x_{0} \in \partial \pi_{p}\right|_{C}(C)-\left.\pi_{p}\right|_{C}(C)$. Поэтому имеется последовательность точек $a_{i} \in D_{p q} \cap C$ такая, что $a_{i} \rightarrow a_{*} \in D_{p q}-C$ и $\left.\pi_{p}\right|_{C}\left(a_{i}\right) \rightarrow x_{0}$ при $i \rightarrow \infty$.

Пусть $U\left(x_{0}\right) \subset W^{u}(p)-\operatorname{int} D_{p}$ - окрестность точки $x_{0}$ в $W^{u}(p)-\operatorname{int} D_{p}$, удовлетворяюшая шагу 4.1. Тогда окрестность $U=\pi_{p}^{-1}\left(U\left(x_{0}\right)\right)$ заполнена неустойчивыми $n$-мерными и $(n-1)$-мерными инвариантными многообразиями, необходимо пересекающими $\pi_{p}^{-1}\left(x_{0}\right)$. Так как $a_{i} \rightarrow a_{*}$, то $a_{i} \in U$ начиная с некоторого индекса $i_{0}$. Следовательно, $C$ пересекает $\pi_{p}^{-1}\left(x_{0}\right)$. Но тогда $\left.x_{0} \notin \partial \pi_{p}\right|_{C}(C)$, поскольку $\left.\pi_{p}\right|_{C}$ является локальным гомеоморфизмом. Это противоречие завершает доказательство.

ШАГ 4.4. Если $n \geqslant 4$, то $\left.\pi_{p}\right|_{C}: C \rightarrow W^{u}(p)-\operatorname{int} D_{p}-$ гомеоморфизм и $\pi_{p}^{-1}(x) \cap C$ coстоит из одной точки для любого $x \in W^{u}(p)-\operatorname{int} D_{p} . \quad B$ частности, $\left.P\right|_{S}: S \rightarrow S_{p}^{n-2}$ есть гомеоморфизм и $S$ является $(n-2)$-мерной сферой.

ДокаЗАТЕЛьСтво. Из шагов 4.2 и 4.3 вытекает, что $\left.\pi_{p}\right|_{C}: C \rightarrow W^{u}(p)-$ int $D_{p}$ - накрытие. Так как $n \geqslant 4$, то $\pi_{1}\left(S_{p}^{n-2}\right)=\pi_{1}\left(W^{u}(p)-\operatorname{int} D_{p}\right)=0$. Поэтому $\left.\pi_{p}\right|_{C}: C \rightarrow W^{u}(p)-\operatorname{int} D_{p}$ является тривиальным накрытием (см., например, [30, п. 13.9]), т.е. $C$ гомеоморфно произведению множества $W^{u}(p)-\operatorname{int} D_{p}$ и не более чем счетного множества точек. Из связности $C$ вытекает, что слой накрытия $\left.\pi_{p}\right|_{C}$ состоит из одной точки. Поэтому $\left.\pi_{p}\right|_{C}$ есть гомеоморфизм $C \rightarrow W^{u}(p)-\operatorname{int} D_{p}$. Из определения $\left.\pi_{p}\right|_{C}$ получаем, что $\left.S \cap \pi_{p}^{-1}(x)\right|_{C}$ состоит из одной точки для каждого $x \in W^{u}(p)-\operatorname{int} D_{p}$. 
Перед доказательством аналогичного утверждения для $n=3$ покажем, что некоторое специальное семейство кривых на цилиндре $C_{p q}$ удовлетворяет теореме о непрерывной зависимости от начальных условий. Напомним, что из сильного условия трансверсальности следует, что цилиндр $C_{p q}$ пересекается только 2-мерными и 3 -мерными неустойчивыми инвариантными многообразиями при $n=3$. Обозначим через $D_{2} \subset C_{p q}$ множество пересечений $C_{p q}$ с 2-мерными неустойчивыми инвариантными многообразиями. Эти многообразия пересекают $C_{p q}$ вдоль кривых, семейство которых обозначим через $\mathscr{F}$.

ШАГ 4.5. Кривые семейства $\mathscr{F}$ удовлетворяют теореме о непрерывной зависимости от начальных условий.

ДокАЗАТЕЛЬСтво. Возьмем произвольную кривую $c_{0} \in \mathscr{F}$ и точки $x_{0}, y_{0} \in c_{0}$. Обозначим через $s_{x}$ (соответственно, $s_{y}$ ) слой расслоения $\pi_{p}$, проходящий через $x_{0}$ (соответственно, $\left.y_{0}\right)$; см. рис. 11. Пусть $x_{k} \in D_{2}$ - произвольная последовательность точек, сходяшаяся к $x_{0}$ при $k \rightarrow \infty$. Обозначим через $c_{k} \in \mathscr{F}$ кривую, проходящую через $x_{k}$. Согласно шагу 4.3 каждая кривая $c_{k}$ пересекает $s_{x}$ и $s_{y}$. Поэтому, не уменьшая обшности, можно считать, что $x_{k} \in s_{x}$ и точки $x_{k}$ сходятся к $x_{0}$ монотонно на $s_{x}$. Пусть $\left[x_{k}, y_{k}\right] \subset c_{k}$ - отрезок с конщевыми точками $x_{k}$ и $y_{k} \in s_{y}$ такими, что $\pi_{p}\left(\left[x_{k}, y_{k}\right]\right)=\pi_{p}\left(\left[x_{0}, y_{0}\right]\right)$ для любого $k$ (см. рис. 11$)$, где отрезок $\left[x_{0}, y_{0}\right] \subset c_{0}$ отображается посредством $\pi_{p}$ в $\pi_{p}\left(\left[x_{0}, y_{0}\right]\right)$ гомеоморфно. Тогда последовательность $y_{k}$ монотонна на $s_{y}$. Мы должны доказать, что $y_{k} \rightarrow y_{0}$ при $k \rightarrow \infty$.

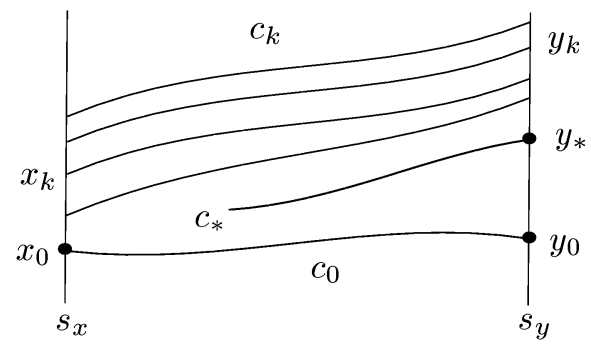

Рис. 11. Семейство $\mathscr{F}$

Предположим противное. Тогда, перейдя, если необходимо, к подпоследовательности, можно считать, что $y_{k} \rightarrow y_{*}$ при $k \rightarrow \infty$ для некоторой точки $y_{*} \in s_{y}$, лежащей между $y_{1}$ и $y_{0}, y_{*} \neq y_{0}$. Так как $D_{2}$ - компакт, то $y_{*} \in D_{2}$ и, следовательно, существует кривая, скажем $c_{*} \in \mathscr{F}$, проходящая через $y_{*}$. В силу шага $4.3 c_{*}$ пересекает $s_{x}$. Возьмем отрезок $\left[x_{*}, y_{*}\right] \subset c_{*}$ такой, что $\pi_{p}\left(\left[x_{*}, y_{*}\right]\right)=\pi_{p}\left(\left[x_{0}, y_{0}\right]\right)$, где $x_{*} \in s_{x}$. Тогда $x_{*} \neq x_{0}$, поскольку $y_{*} \neq y_{0}$. Это невозможно, потому что последовательность $y_{k}$ монотонная на $s_{y}$ и, следовательно, нет точек вида $y_{k}$ на отрезке $\left[y_{*}, y_{0}\right] \subset s_{y}$ между $y_{*}, y_{0}$.

ШАГ 4.6. Каждая компонента $S$ пересечения $C \cap C_{p q}$ является $(n-2)$-мерной сферой. Более того, $S \cap P^{-1}(x)$ состоит из одной точки для каждого $x \in S_{p}^{n-2}$. 
ДОКАЗАТЕЛЬСТво. Согласно шагу 4.4 осталось рассмотреть только случай $n=3$. Переобозначим $S=c$ и предположим, что кривая $c \subset W^{u}\left(z^{\prime}\right) \cap C_{p q}$ не гомеоморфна окружности. Тогда $c$ имеет точку накопления $m_{0} \in C_{p q}$, т.е. сушествует последовательность точек $m_{i} \in c$ таких, что $m_{i} \rightarrow m_{0}$ при $i \rightarrow \infty$. Возьмем окрестность $U\left(m_{0}\right) \subset C_{p q}$ точки $m_{0}$, которая является тривиальным расслоением над отрезком $P\left(U\left(m_{0}\right)\right), P\left(m_{0}\right) \in P\left(U\left(m_{0}\right)\right)$, со слоями, являющимися дугами устойчивых многообразий точек $x \in P\left(U\left(m_{0}\right)\right)$ (см. рис. 12$)$.

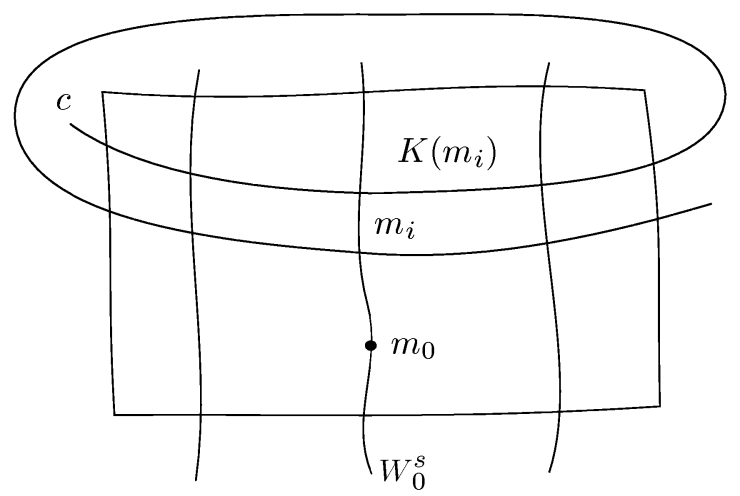

Рис. 12. Окрестность $U\left(m_{0}\right)$ точки $m_{0}$

Пусть $W_{0}^{s} \subset U\left(m_{0}\right)$ - компонента множества $W^{s}\left(P\left(m_{0}\right)\right) \cap U\left(m_{0}\right)$, проходящая через $m_{0}$. Обозначим через $K\left(m_{i}\right)$ компоненту множества $c \cap U\left(m_{0}\right)$, содержащую точку $m_{i}$. В силу шага 4.3 можно считать, что $K\left(m_{i}\right)$ пересекает $W_{0}^{s}$. Так как $K\left(m_{i}\right)$ трансверсально слоям тривиального расслоения $U\left(m_{0}\right)$, то $K\left(m_{i}\right) \cap W_{0}^{s}$ состоит из одной точки. Не уменьшая обшности, можно считать, что $K\left(m_{i}\right) \cap W_{0}^{s}=$ $m_{i}$. Более того, можно считать, что компоненты $K\left(m_{i}\right)$ попарно не пересекаются, поскольку $m_{i} \rightarrow m_{0}$ при $i \rightarrow \infty$.

Обозначим через $C\left(m_{i}, m_{i+1}\right)$ дугу кривой $c$ с конщевыми точками $m_{i}, m_{i+1}$ и через $C_{i, i+1}$ - замкнутую кривую, равную объединению $C\left(m_{i}, m_{i+1}\right)$ и отрезка $\left(m_{i}, m_{i+1}\right) \subset W_{0}^{s}$. Так как $C\left(m_{i}, m_{i+1}\right)$ трансверсальна слоям расслоения $P: \operatorname{clos} C_{p q} \rightarrow S_{p}^{n-2}$, то $P\left(m_{i}\right)=P\left(m_{i+1}\right), C_{i, i+1}$ не гомотопна нулю в цилиндре $\cos C_{p q}$ и $P\left(C_{i, i+1}\right)=S_{p}^{n-2}$. В силу сильного условия трансверсальности $C\left(m_{i}, m_{i+1}\right)$ пересекает $P^{-1}\left(P\left(m_{0}\right)\right)$ только в точках $m_{i}, m_{i+1}$. Отсюда вытекает, что последовательность $\left\{m_{i}\right\}_{i=1}^{\infty}$ монотонно сходится к $m_{0}$ и, следовательно, $c$ спиралеобразно подходит к $m_{0}$. Согласно [45] и [66] $m_{0} \in W^{u}(\theta)$ для некоторой точки $\theta$ базисного множества $\Theta$. В силу сильного условия трансверсальности $\operatorname{dim} W^{u}(\theta) \geqslant 2$. Так как $m_{0}$ - точка накопления точек $m_{i} \in W^{u}\left(z^{\prime}\right)$ с $\operatorname{dim} W^{u}\left(z^{\prime}\right)=2$, то $\operatorname{dim} W^{u}(\theta)=2$. Поэтому пересечение $W^{u}(\theta) \cap \operatorname{clos} C_{p q}$ содержит кривую $c_{0}$, проходящую через $m_{0}$.

Согласно шагу 4.5 кривая $c_{0}$ принадлежит предельному множеству кривой $c$. Аргументы теории Пуанкаре-Бендиксона (см., например, [33], [6]) с использованием того, что $c$ и $c_{0}$ трансверсальны дугам $P^{-1}(a), a \in S_{p}^{1}$, показывают, что $c_{0}-$ замкнутая кривая. Следовательно, $c_{0}$ ограничивает диск на $W^{u}(\theta)$.

Покажем, что $\theta$ - периодическая точка. Согласно теореме о непрерывной зависимости инвариантных многообразий от начальных условий существует $\alpha>0$ та- 
кое, что любое неустойчивое многообразие $W^{u}(\eta), \eta \in \Theta$, пересекающее $W_{\alpha}^{s}(\theta)$, необходимо пересекает цилиндр $\operatorname{clos} C_{p q}$ вдоль замкнутой кривой вблизи $c_{0}$. Так как $c$ спиралеобразно подходит к $c_{0}$, то, по крайней мере, одна компонента множества $W_{\alpha}^{s}(\theta)-\theta$ не пересекается с неустойчивыми многообразиями $W^{u}(\eta), \eta \in \Theta$. Тогда из предложения 1.1 получаем, что $\theta$ - периодическая точка.

Таким образом, для достаточно большого $\beta>0$ диск $W_{\beta}^{u}(\theta)$ пересекает цилиндр $\cos C_{p q}$ вдоль замкнутой кривой $c_{0}$. Аналогично доказательству леммы 1.5 из [56] можно показать, что $W^{u}\left(z^{\prime}\right)$ пересекает $W_{\alpha}^{s}(\theta)-\theta$. Тогда из $\lambda$-леммы и теоремы о непрерывной зависимости инвариантных многообразий от начальных условий вытекает, что $W^{u}\left(z^{\prime}\right)$ пересекает цилиндр $\operatorname{clos} C_{p q}$ вблизи $m_{0}$ вдоль замкнутых кривых. Полученное противоречие показывает, что $c$ - замкнутая кривая.

Для завершения доказательства осталось заметить, что из замкнутости $c$ и сильного условия трансверсальности вытекает, что пересечение $c \cap P^{-1}(x)$ состоит из одной точки для любого $x \in S_{p}^{1}$.

Отметим, что в шагах $4.2-4.4$ и 4.6 сфера $S_{p}^{n-2}$ могла быть любой цилиндрически вложенной в $W^{u}(p)(n-2)$-мерной сферой, не проходящей через точку $p$. Все, что нам было необходимо при доказательстве этих шагов, это только сушествование множества $\pi_{p}^{-1}\left(S_{p}^{n-2}\right) \stackrel{\text { def }}{=} \operatorname{clos} C_{p q}$. В дальнейшем будем рассматривать сфферу $S_{p}^{n-2}$ как границу $(n-1)$-мерного шара $D_{p}$, принадлежащего характеристической cф̆epe $S_{p q}$.

ШАГ 4.7. ( $n-2)$-мерная сфера $S=C \cap C_{p q}$ изотопна сферам $S_{p}^{n-2} u$ $S_{q}^{n-2}$ в чилиндре $\operatorname{clos} C_{p q}$. $B$ частности, включение $S \subset D_{p q}$ индуцирует мономорфизм $\pi_{n-2}(S) \rightarrow \pi_{n-2}\left(D_{p q}\right) \cong \mathbb{Z}$. Более того, $S$ разбивает $\operatorname{clos} C_{p q}$ на два иилиндра, каждый из которых гомеоморфен $\Sigma^{n-2} \times[0 ; 1]$.

ДоказАтельство. Напомним, что $P$ есть тривиальное расслоение со слоем [0;1]. В силу шага 4.3 и сильного условия трансверсальности $\left.P\right|_{S}: S \rightarrow S_{p}^{n-2}-$ гомеоморфизм. В частности, каждый слой расслоения $\left(\operatorname{clos} C_{p q}, S_{p}^{n-2}, P\right)$ пересекает $S$ ровно в одной точке. Поэтому существует изотопия $S$ в $S_{p}^{n-2}$ вдоль слоев расслоения $\left(\operatorname{clos} C_{p q}, S_{p}^{n-2}, P\right)$. Аналогично, $S$ изотопна $S_{q}^{n-2}$. Tак как $S$ пересекает каждый слой тривиального расслоения $\left(\operatorname{clos} C_{p q}, S_{p}^{n-2}, P\right)$ ровно в одной точке, а слоем является интервал [0;1], то $S$ разбивает $\operatorname{clos} C_{p q}$ на два цилиндра, каждый из которых гомеоморфен $\Sigma^{n-2} \times[0 ; 1]$. Отсюда и из следствия 2.3 получаем, что $\pi_{n-2}(S) \rightarrow \pi_{n-2}\left(D_{p q}\right)$ - мономорфизм.

В силу теоремы о непрерывной зависимости инвариантных многообразий от начальных условий каждая $(n-2)$-мерная сфера $S$ из пересечения $W^{u}\left(z^{\prime}\right) \cap C_{p q}$ является цилиндрически вложенной как в $W^{u}\left(z^{\prime}\right)$, так и в $C_{p q}$. Поэтому $S$ ограничивает открытый $(n-1)$-мерный шар в $W^{u}\left(z^{\prime}\right)$, который мы обозначим через $B_{S}^{n-1}$ [36]. Такая $(n-2)$-мерная сфера $S \subset W^{u}\left(z^{\prime}\right) \cap C_{p q}$ и соответствующий $(n-1)$-мерный шар $B_{S}^{n-1} \subset W^{u}\left(z^{\prime}\right)$ называются минимальнымми, если внутри $B_{S}^{n-1}$ нет точек из $W^{u}\left(z^{\prime}\right) \cap C_{p q}$. В силу сильного условия трансверсальности любое компактное подмножество $K \subset W^{u}\left(z^{\prime}\right)$ пересекает конечное число сфер из $W^{u}\left(z^{\prime}\right) \cap C_{p q}$. Поэтому минимальные сферы и соответствуюшие минимальные шары сушествуют.

ШАГ 4.8. Пусть $B_{S}^{n-1} \subset W^{u}\left(z^{\prime}\right)$ - минимальныц $(n-1)$-мерный шар, ограниченный минимальной $(n-2)$-мерной сферой $S \subset W^{u}\left(z^{\prime}\right) \cap C_{p q}$. Тогда $B_{S}^{n-1} \cap D_{p q}=\varnothing$. 
ДокАЗАТЕЛЬСтво. Предположим противное. Тогда $B_{S}^{n-1} \subset D_{p q}$, поскольку шар $B_{S}^{n-1}$ минимальный. Поэтому $S$ определяет тривиальный элемент группы $\pi_{n-2}\left(D_{p q}\right)$. С другой стороны, согласно шагу 4.7 сфера $S$ определяет нетривиальный элемент группы $\pi_{n-2}\left(D_{p q}\right)$. Полученное противоречие завершает доказательство.

Имеются следуюшие возможности:

1) существует минимальный $(n-1)$-мерный шар $B^{\prime} \subset W^{u}\left(z^{\prime}\right)$, содержащий точку $z^{\prime}$

2) не существует минимального шара, содержащего точку $z^{\prime}$.

Рассмотрим случай 1$)$. Не уменьшая общности, можно считать, что $f\left(z^{\prime}\right)=z^{\prime}$, $f(p)=p$ и $f(q)=q$, иначе мы перешли бы к некоторой итерации $f^{k}$. Более того, будем предполагать, что ограничение $\left.f\right|_{W^{u}\left(z^{\prime}\right)}$ является сохраняющим ориентацию диффеоморфизмом.

Напомним, что гомеоморфизм $\psi: M \rightarrow M$ является устойчивылм в cмbысле Бинга (см., например, [18]), если $\psi$ можно представить в виде суперпозиции $\psi=\psi_{1} \circ \cdots \circ \psi_{k}$, где каждый гомеоморфизм $\psi_{i}, 1 \leqslant i \leqslant k$, неподвижен внутри некоторого открытого множества $D_{i} \subset M$. Известно, что сохраняющий ориентацию диффеоморфизм устойчив в смысле Бинга.

Так как $f\left(C_{p q}\right) \subset D_{p q}$, то $f(S) \cap S=\varnothing$, где $(n-2)$-мерная сфрера $S$ ограничивает $B^{\prime}$ на $W^{u}\left(z^{\prime}\right)$. Поэтому $S$ лежит внутри $f(S)$, поскольку $z^{\prime} \in B^{\prime}$ является гиперболическим источником ограничения $\left.f\right|_{W^{u}\left(z^{\prime}\right)}$. Тогда согласно [37] $(n-2)$-мерные сферы $S, f(S)$ ограничивают замкнутое кольцо, обозначаемое через $A \subset W^{u}\left(z^{\prime}\right)$, гомеоморфное $\Sigma^{n-2} \times[0 ; 1]$, так как диффеоморфизм $\left.f\right|_{W^{u}\left(z^{\prime}\right)}$ устойчив в смысле Бинга. Учитывая $f(S) \cap S=\varnothing$, получаем, что $A$ есть фундаментальное кольцо ограничения $\left.f\right|_{W^{u}\left(z^{\prime}\right)}$, т.е.

$$
\bigcup_{j \in \mathbb{Z}} f^{j}(A)=W^{u}\left(z^{\prime}\right)-z^{\prime}, \quad \operatorname{int} f^{i}(A) \cap \operatorname{int} f^{l}(A)=\varnothing, \quad i \neq l .
$$

Более того, $\bigcup_{j \geqslant 0} f^{j}(A)=W^{u}\left(z^{\prime}\right)-B^{\prime}$. В частности, $B^{\prime} \cap A=\varnothing$.

ШАГ 4.9. Предположим, что имеет место случай 1), т.е. S-минимальная сфера, содержащая внутри $z^{\prime}$, и пусть $A$ - фундаментальное кольцо диффеоморфизма $\left.f\right|_{W^{u}\left(z^{\prime}\right)}$, ограниченное $(n-2)$-мерными сферами $S, f(S)$. Тогда

$$
A \subset D_{p q}, \quad A \cap \operatorname{int} D_{p q}=\operatorname{int} A \cup f(S)
$$

ДокАЗАТЕЛЬСТво. Пусть $h: \Sigma^{n-2} \times[0 ; 1] \rightarrow A-$ произвольньй сохраняющий ориентацию гомеоморфизм такой, что

$$
h\left(\Sigma^{n-2} \times\{0\}\right)=S \text { и } h\left(\Sigma^{n-2} \times\{1\}\right)=f(S) .
$$

В силу сильного условия трансверсальности ограничение

$$
\left.P_{A} \stackrel{\text { def }}{=} \pi_{p}\right|_{A \cap D_{p q}}: A \cap D_{p q} \rightarrow W^{u}(p)-\operatorname{int} D_{p}
$$




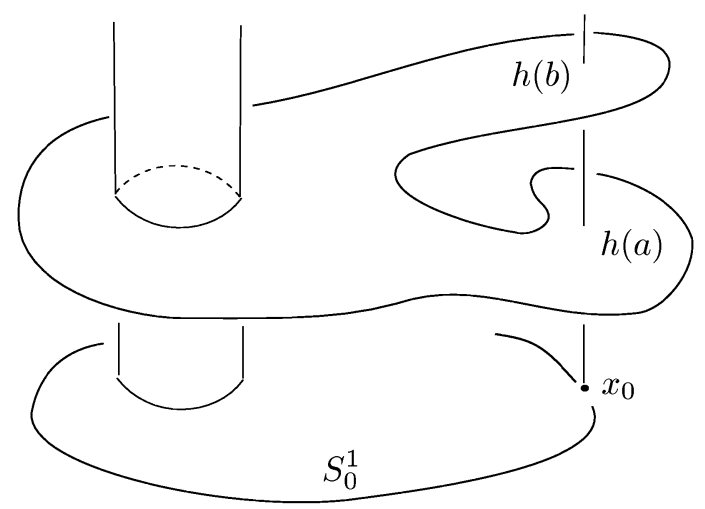

Рис. 13. $P_{A}(h(a))=P_{A}(h(b))=x_{0}$

является локальным гомеоморфизмом. Так как $B^{\prime} \cap D_{p q}=\varnothing($ см. шаг 4.8) и $B^{\prime} \cap A=\varnothing$, то сушествует $\varepsilon>0$ такое, что $h\left(\Sigma^{n-2} \times[0 ; \varepsilon]\right) \subset D_{p q}$. Поэтому $P_{A}\left(h\left(\Sigma^{n-2} \times[0 ; \varepsilon]\right)\right) \subset W^{u}(p)-\operatorname{int} D_{p}$. Кроме того, можно взять $\varepsilon>0$ таким, чтобы $P_{A}$ переводил кольцо $h\left(\Sigma^{n-2} \times[0 ; \varepsilon]\right)$ на $P_{A}\left(h\left(\Sigma^{n-2} \times[0 ; \varepsilon]\right)\right)$ гомеоморфно, поскольку $P_{A}$ является локальньм гомеоморфизмом, а $\left.P_{A}\right|_{S}=\left.P\right|_{S}$ является гомеоморфизмом $S \rightarrow S_{p}^{n-2}$ (см. шаг 4.6). Следовательно, для любого $0 \leqslant t<\varepsilon(n-2)$-мерная сфера $P_{A}\left(h\left(\Sigma^{n-2} \times\{t\}\right)\right)$ лежит внутри $(n-2)$-мерной сферы $P_{A}\left(h\left(\Sigma^{n-2} \times\{\varepsilon\}\right)\right)$ на неустойчивом многообразии $W^{u}(p)$.

Обозначим через $E \subset[0 ; 1]$ множество параметров $t_{0}$, удовлетворяюших следующим условиям:

a) $h\left(\Sigma^{n-2} \times\left[0 ; t_{0}\right]\right) \subset D_{p q}$ и $P_{A}\left(h\left(\Sigma^{n-2} \times\left[0 ; t_{0}\right]\right)\right) \subset W^{u}(p)-\operatorname{int} D_{p}$;

б) $P_{A}$ гомеоморфно отображает $(n-1)$-кольцо $h\left(\Sigma^{n-2} \times\left[0 ; t_{0}\right]\right)$ на $P_{A}\left(h\left(\Sigma^{n-2} \times\right.\right.$ $\left.\left.\left[0 ; t_{0}\right]\right)\right)$;

в) для любого $0 \leqslant t<t_{0}(n-2)$-мерная сфера $P_{A}\left(h\left(\Sigma^{n-2} \times\{t\}\right)\right)$ лежит внутри $(n-2)$-мерной сферы $P_{A}\left(h\left(\Sigma^{n-2} \times\left\{t_{0}\right\}\right)\right)$ на $W^{u}(p)$; в частности, $P_{A}\left(h\left(\Sigma^{n-2} \times\right.\right.$ $\left.\left.\left[0 ; t_{0}\right]\right)\right) \subset W^{u}(p)-\operatorname{int} D_{p}$.

Множество $E$ непусто, поскольку $[0 ; \varepsilon] \subset E$. В силу сильного условия трансверсальности $E$ открыто в [0;1]. Далее, если $t \in E$, то $[0, t] \subset E$. Таким образом, $E$ есть промежуток в $[0 ; 1]$ с левой концевой точкой 0 . Пусть $t_{*}=\sup E$. Очевидно, $t_{*}>0$. В силу непрерывности гомеоморфизма $h$ имеем $h\left(\Sigma^{n-2} \times\left\{t_{*}\right\}\right) \subset D_{p q}$, так как $D_{p q}$ замкнуто. Более того, $h\left(\Sigma^{n-2} \times\left\{t_{*}\right\}\right) \subset \operatorname{int} D_{p q}$, поскольку $[0 ; \varepsilon] \subset E$ при $\varepsilon>0$ и, следовательно, $t_{*} \geqslant \varepsilon$.

Покажем, что $P_{A}$ гомеоморфно отображает $h\left(\Sigma^{n-2} \times\left\{t_{*}\right\}\right)$ на $P_{A}\left(h\left(\Sigma^{n-2} \times\right.\right.$ $\left.\left.\left\{t_{*}\right\}\right)\right)$. Для $n \geqslant 4$ это следует из шага 4.4. Осталось доказать этот факт для $n=3$. Предположим противное. Тогда существуют точки $a, b \in \Sigma^{1} \times\left\{t_{*}\right\}$ такие, что

$$
P_{A}(h(a))=P_{A}(h(b)) \stackrel{\text { def }}{=} x_{0}, \quad a \neq b
$$

(см. рис. 13). Тогда $h\left(\Sigma^{1} \times\left[0 ; t_{*}\right]\right)$ пересекает дугу $\pi_{p}^{-1}\left(x_{0}\right)$ в двух точках $h(a)$, $h(b)$. В силу сильного условия трансверсальности существуют 2-мерные диски $D_{a} \subset W^{u}\left(z^{\prime}\right), D_{b} \subset W^{u}\left(z^{\prime}\right)$, содержащие точки $a, b$ соответственно, такие, что $D_{a} \cap D_{b}=\varnothing$. Более того, можно считать, что $\pi_{p}$ отображает оба диска $D_{a}$ и $D_{b}$ 
гомеоморфно на $\pi_{p}\left(D_{a}\right)$ и $\pi_{p}\left(D_{b}\right)$ соответственно, и $D_{a} \cup D_{b} \subset \operatorname{int} D_{p q}$, поскольку $h(a), h(b) \subset \operatorname{int} D_{p q}$.

Возьмем $0<t<t_{*}$ столь близким к $t_{*}$, чтобы простая замкнутая кривая $h\left(\Sigma^{1} \times\{t\}\right)$ пересекала оба диска $D_{a}$ и $D_{b}$. Так как $P_{A}\left(D_{a}\right)$ и $P_{A}\left(D_{b}\right)$ являются дисками, то $P_{A}\left(h\left(\Sigma^{1} \times\{t\}\right)\right)$ ограничивает диск $B_{0}$ в $W^{u}(p)$,

$$
B_{0} \cup P_{A}\left(\left(D_{a}\right) \cup P_{A}\left(D_{b}\right)\right) \stackrel{\text { def }}{=} Q,
$$

содержащий простую замкнутую кривую $S_{0}^{1}$, проходящую через $x_{0}$. Более того, можно взять $S_{0}^{1}$ так, чтобы $S_{0}^{1} \subset Q-D_{p}$. Тогда $\pi_{p}^{-1}\left(S_{0}^{1}\right)$ определено и гомеоморфно замкнутому кольцу $\Sigma^{1} \times[0 ; 1]$. По построению пересечение $\pi_{p}^{-1}\left(S_{0}^{1}\right) \cap W^{u}\left(z^{\prime}\right)$ содержит компоненту $S_{0}$, проходящую через точки $h(a), h(b)$. Это противоречит шагу 4.6. Таким образом, $P_{A}$ отображает $h\left(\Sigma^{n-2} \times\left\{t_{*}\right\}\right)$ на $P_{A}\left(h\left(\Sigma^{1} \times\left\{t_{*}\right\}\right)\right)$ гомеоморфно для всех $n \geqslant 3$.

Учитывая сильное условие трансверсальности, получаем $t_{*} \in E$. Поэтому $t_{*}=1$. Следовательно, $P_{A}\left(h\left(\Sigma^{n-2} \times[0 ; 1]\right)\right) \subset W^{u}(p)-\operatorname{int} D_{p}$ и $A \cap \operatorname{int} D_{p q}=$ int $A \cup f(S)$.

Положим $W^{\prime}=W^{u}\left(z^{\prime}\right)-B^{\prime}$. Из шага 4.9 следует $W^{\prime} \subset D_{p q} \subset W^{s}(\Omega)$, поскольку $\bigcup_{j \geqslant 0} f^{j}(A)=W^{u}\left(z^{\prime}\right)-B^{\prime}$ и $f^{j}\left(D_{p q}\right) \subset D_{p q}$ для любого $j \geqslant 0$. Поэтому $C=W^{\prime}$ является единственной компонентой пересечения $D_{p q} \cap W^{u}\left(z^{\prime}\right)$, а $S=C \cap$ $C_{p q}$ - единственная компонента пересечения $W^{u}\left(z^{\prime}\right) \cap C_{p q}$. Это доказывает теорему в случае 1$)$.

Рассмотрим случай 2), когда отсутствуют минимальные шары, содержашие $z^{\prime}$. Покажем, что этот случай не осушествляется.

ШАГ 4.10. Предположим, что имеет место случай 2), т.е. не существует минимального шара, содержащего точку $z^{\prime}$. Тогда любой путь $\omega \subset$ $W^{u}\left(z^{\prime}\right)$, соединяющий $z^{\prime}$ и минимальную сферу, пересекает сферы из пересечения $W^{u}\left(z^{\prime}\right) \cap C_{p q}$, при әтом

$$
\operatorname{int} \omega \cap\left(W^{u}\left(z^{\prime}\right) \cap C_{p q}\right) \neq \varnothing .
$$

ДОКАЗАТЕЛЬСтво. Пусть $\omega$ - путь, соединяющий точку $z^{\prime}$ и $z^{\prime \prime} \in S$, где $S \subset$ $W^{u}\left(z^{\prime}\right) \cap C_{p q}$ - некоторая минимальная сфера. Предположим противное. Тогда

$$
\omega \cap\left(W^{u}\left(z^{\prime}\right) \cap C_{p q}\right)=z^{\prime \prime} .
$$

Согласно шагу $4.8 B_{S} \cap D_{p q}=\varnothing$, где $B_{S} \subset W^{u}\left(z^{\prime}\right)$ - минимальный $(n-1)$-мерный шар, ограниченный сферой $S$. Из сильного условия трансверсальности следует, что внешность $S$ вблизи $S$ лежит в $D_{p q}$. Поэтому $\omega \subset D_{p q}$ и, следовательно, $z^{\prime} \subset D_{p q}$, что невозможно.

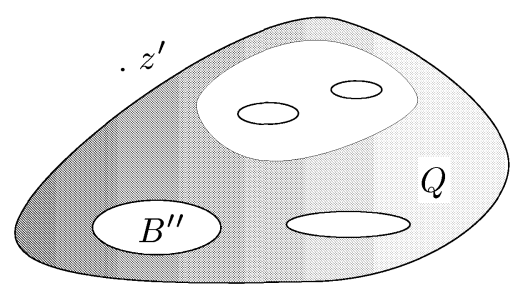

Рис. 14. Область $Q$ 
ШАГ 4.11. Предположим, что имеет место случай 2), т.е. не существует минимального шара, содержащего точку $z^{\prime}$. Тогда существует область $Q \subset W^{u}\left(z^{\prime}\right)$, ограниченная сферами из $W^{u}\left(z^{\prime}\right) \cap C_{p q}$, такая, что $Q \subset D_{p q}, n p u$ этом $Q$ лежит внутри некоторой сферы $S_{\mathrm{ext}} \subset W^{u}\left(z^{\prime}\right) \cap C_{p q}$ (cм. рис. 14).

ДокаЗАтЕльство. Достаточно показать, что существуют сфера $S^{\prime} \subset$ $W^{u}\left(z^{\prime}\right) \cap C_{p q}$ и минимальньй шар $B^{\prime \prime} \subset W^{u}\left(z^{\prime}\right)$ внутри $S^{\prime}$ такие, что нет сфер из $W^{u}\left(z^{\prime}\right) \cap C_{p q}$, лежащих внутри $S^{\prime}$ и содержащих внутри себя $B^{\prime \prime}$. Действительно, в этом случае рассмотрим область $R \subset W^{u}\left(z^{\prime}\right)$, ограниченную $S^{\prime} \stackrel{\text { def }}{=} S_{\text {ext }}$, и затем удалим из $R$ все шары, ограниченные сферами из $W^{u}\left(z^{\prime}\right) \cap C_{p q}$, которые лежат внутри $S^{\prime}$. Тогда получим требуемую область $Q$, поскольку $Q$ содержит часть внешности шара $B^{\prime \prime}$, которая согласно шагу 4.8 лежит в $D_{p q}$ вблизи $B^{\prime \prime}$ и, следовательно, $Q \subset D_{p q}$, так как внутренность $Q$ не содержит сфер из $W^{u}\left(z^{\prime}\right) \cap C_{p q}$.

Возьмем произвольный минимальный шар $B^{\prime} \subset W^{u}\left(z^{\prime}\right)$. Согласно шагу 4.10 существует сфера $S \subset W^{u}\left(z^{\prime}\right) \cap C_{p q}$, отделяющая $z^{\prime}$ от $B^{\prime}$. Тогда либо $z^{\prime}$, либо $B^{\prime}$ лежит внутри $S$. В последнем случае достаточно взять минимально возможную сферу, которая ограничивает область с шаром $B^{\prime}$ и не является границей шара $B^{\prime}$. Из сильного условия трансверсальности и шагов $4.8,4.10$ такая сфера существует.

Таким образом, осталось рассмотреть случай, когда точка $z^{\prime}$ лежит в шаре $B$, ограниченном сферой $S$. Из сильного условия трансверсальности и компактности $B \cup S$ следует, что шар $B$ содержит конечное число сфер из $W^{u}\left(z^{\prime}\right) \cap C_{p q}$. Поэтому, перейдя к минимально возможной, если необходимо, сфере, можно считать, что внутри $S$ нет других сфер, окружаюших $z^{\prime}$. Так как никакой минимальный шар согласно шагу 4.8 не содержит $z^{\prime}$, то $B$ не является минимальным. Следовательно, $B$ содержит минимальный шар, скажем $B^{\prime \prime}$, ограниченньй сферой $S^{\prime \prime}=\partial B^{\prime \prime}$, также лежашей внутри $S$. Согласно шагу 4.8 имеем $B^{\prime \prime} \cap D_{p q}=\varnothing$. Очевидно, $z^{\prime} \notin D_{p q}$. Поэтому в силу шага 4.10 сушествует сфера, окружающая $B^{\prime \prime}$, но не окружаюшая $z^{\prime}$. Мы приходим к случаю, рассмотренному вьше. Это завершает доказательство.

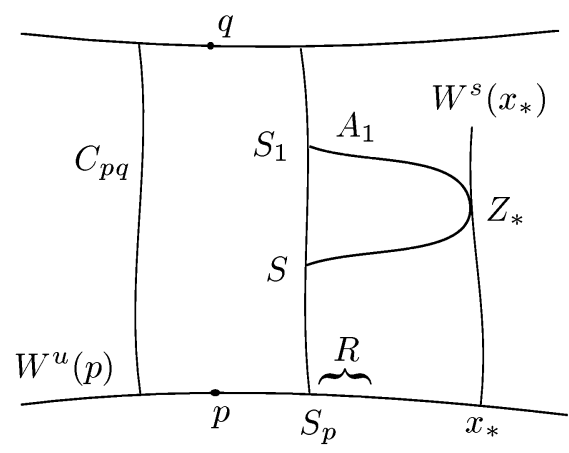

Рис. 15. Точка $z_{*}$ нарушения локальной гомеоморфности $P_{Q}$

Обозначим через $Q \subset W^{u}\left(z^{\prime}\right)$ замыкание области, удовлетворяюшей условиям шага 4.11. Тогда $Q \cap C_{p q}=Q \cap D_{p q}=\partial Q$, поскольку $\partial Q \subset W^{u}\left(z^{\prime}\right) \cap C_{p q}$. Положим

$$
\left.P_{Q} \stackrel{\text { def }}{=} \pi_{p}\right|_{Q}: Q \rightarrow W^{u}(p)-\operatorname{int} D_{p} .
$$


Из сильного условия трансверсальности следует, что $\left.P_{Q}\right|_{\text {int }} Q$ является локальным гомеоморфизмом. Поэтому

$$
\left.P_{Q}\right|_{\text {int } Q}: \operatorname{int} Q \rightarrow P_{Q}(\operatorname{int} Q)
$$

является накрытием. Снова применяя сильное условие трансверсальности, получаем, что сушествует кольцо $A_{1} \subset Q$ с $\partial A_{1} \subset \partial Q$ такое, что кольцо $P_{Q}\left(A_{1}\right)=R$ имеет $S_{p}$ в качестве одной из компонент границы (см. рис. 15). Отсюда вытекает, что $\partial P_{Q}(Q)-S_{p} \neq \varnothing$, поскольку $Q$ и $P_{Q}(Q)$ - компактные множества. Возьмем произвольную точку $x_{*} \in \partial P_{Q}(Q)-S_{p}$. Сушествует точка $z_{*} \in Q$ с $P_{Q}\left(z_{*}\right)=x_{*}$, так как $P_{Q}(Q)$ - компакт. Поэтому $\partial P_{Q}(Q) \subset P_{Q}(Q)$. Так как $x_{*} \in \partial P_{Q}(Q)-S_{p}$, то $z_{*} \in \operatorname{int} Q$. Отсюда вытекает, что $P_{Q} \mid \operatorname{int} Q$ не является локальным гомеоморфизмом в точке $z_{*}$, что противоречит сильному условию трансверсальности. Это противоречие завершает доказательство теоремы.

Основной результат этого параграф̆а содержится в следующей теореме.

ТЕОРема 4.2. Пусть $f$ - A-диффеоморфизм замкнутого п-мерного многообразия $M^{n}, \quad n \geqslant 3$, и $\Omega$ - ориентируемый растягивающийся аттрактор коразмерности один. Предположим, что имеется нетривиальное базисное множество $\Omega^{\prime} \neq \Omega$ диффеоморфизма $f$ такое, что $W^{u}\left(\Omega^{\prime}\right) \cap W^{s}(\Omega) \neq \varnothing$. Тогда $f$ не является структурно устойчивым.

ДокАЗАТЕльство. Согласно результатам [51], [62] достаточно показать, что $f$ не удовлетворяет сильному условию трансверсальности. Поэтому можно считать, что индекс Морса любого базисного множества $\Theta$, удовлетворяющего условию $W^{u}(\Theta) \cap W^{s}(\Omega) \neq \varnothing$, не меньше чем $n-1$, поскольку $\operatorname{dim} E_{x}^{s}=1, x \in \Omega$.

Предположим, что теорема неверна. Тогда любое устойчивое многообразие $W^{s}(x), x \in \Omega$, пересекается трансверсально с любым неустойчивым многообразием $W^{u}(y), y \in N W(f)$. В силу [45] и условия теоремы имеем $W^{s}(z) \cap W^{u}\left(z^{\prime}\right) \neq \varnothing$ для некоторых точек $z \in \Omega, z^{\prime} \in \Omega^{\prime}$. Поэтому неустойчивое многообразие $W^{u}\left(z^{\prime}\right)$ либо $(n-1)$-мерное, либо $n$-мерное. Если $W^{u}\left(z^{\prime}\right) n$-мерное, то $z^{\prime}$ является периодической точкой, а базисное множество $\Omega^{\prime}$ тривиально. Следовательно, $W^{u}\left(z^{\prime}\right)$ $(n-1)$-мерное. Не уменьшая общности, можно считать, что точки $z$ и $z^{\prime}$ периодические, поскольку периодические точки плотны в базисных множествах.

Возьмем произвольную точку $a \in W^{s}(z) \cap W^{u}\left(z^{\prime}\right)$. Очевидно, каждая точка из $W^{s}(z) \cap W^{u}\left(z^{\prime}\right)$ не принадлежит $\Omega$, поскольку $\Omega$ - аттрактор и состоит из неустойчивых многообразий своих точек. Из предложения 1.4 следует, что либо $a \in\left(x_{a}, y_{a}\right)_{\varnothing}^{s} \subset W^{s}(z)$, где $x_{a} \in W^{u}(p), y_{a} \in W^{u}(q)$ для некоторых ассоциированных граничных периодических точек $p, q \in \Omega$, либо $a \in W_{\varnothing}^{s}(p)$ для некоторой граничной периодической точки $p \in \Omega$. В силу теоремы о непрерьвной зависимости инвариантных многообразий от начальных условий можно считать, что $a \in\left(x_{a}, y_{a}\right)_{\varnothing}^{s} \subset W^{s}(z)$.

Пусть $S_{p q}$ - характеристическая сфера, соответствуюшая 2-связке $W^{u}(p) \cup$ $W^{u}(q)$. Напомним, что $S_{p q}$ есть объединение двух замкнутых $(n-1)$-мерных шаров $D_{p} \subset W^{u}(p), D_{q} \subset W^{u}(q)$ и связывающего цилиндра

$$
C_{p q}=\bigcup_{x \in \partial D_{p}, y \in \partial D_{q}}(x, y)_{\varnothing}^{s}, \quad p \in \operatorname{int} D_{p}, \quad q \in \operatorname{int} D_{q}
$$


В силу построения характеристической сферы (см. п. 2.1) можно взять $S_{p q}$ так, чтобы

$$
\left(x_{a}, y_{a}\right)_{\varnothing}^{s} \subset D_{p q}, \quad D_{p q}=\bigcup_{x \in W^{u}(p)-\operatorname{int} D_{p}}\left[x, \varphi_{p q}(x)\right]_{\varnothing}^{s} .
$$

Тогда $D_{p q} \cap W^{u}\left(z^{\prime}\right) \neq \varnothing$. Согласно следствию $2.4 W^{u}\left(z^{\prime}\right)$ пересекает $C_{p q}$.

Согласно теореме 4.1 пересечение $W^{u}\left(\Omega^{\prime}\right) \cap D_{p q}$ состоит ровно из одной компоненты $C \subset D_{p q} \cap W^{u}\left(z^{\prime}\right)$. Более того,

$$
W^{u}\left(z^{\prime}\right) \cap C_{p q}=C \cap C_{p q}=S^{n-2}
$$

есть $(n-2)$-мерная сфера, изотопная обеим сферам $S_{p}=\partial D_{p}$ и $S_{q}=\partial D_{q}$ в цилиндре $C_{p q}$. Обозначим через $B^{\prime} \subset W^{u}\left(z^{\prime}\right)$ открытьй $(n-1)$-мерный шар, ограниченный $S^{n-2}$ в $W^{u}\left(z^{\prime}\right)$.

Положим $W^{\prime}=W^{u}\left(z^{\prime}\right)-B^{\prime}$, и пусть $\Omega_{c}^{\prime}-C$-плотная компонента базисного множества $\Omega^{\prime}$, содержащая точку $z^{\prime}$. Не уменьшая общности, можно считать, что $f\left(\Omega_{c}^{\prime}\right)=\Omega_{c}^{\prime}$ (см. [2], [35]). Так как $W^{u}\left(z^{\prime}\right) \cap C_{p q}=S^{n-2}$, то $W^{\prime} \subset D_{p q} \subset W^{s}(\Omega)$. Следовательно, $W^{\prime} \cap \Omega_{c}^{\prime}=\varnothing$. Из нетривиальности базисного множества $\Omega^{\prime}$ и $C$-плотности $\Omega_{c}^{\prime}$ вытекает, что пересечение $W^{u}\left(z^{\prime}\right) \cap \Omega_{c}^{\prime}$ плотно в $\Omega_{c}^{\prime}$. Поэтому пересечение

$$
W^{u}\left(z^{\prime}\right) \cap \Omega_{c}^{\prime}=\left(B^{\prime} \cup W^{\prime}\right) \cap \Omega_{c}^{\prime}=B^{\prime} \cap \Omega_{c}^{\prime}
$$

также плотно в $\Omega_{c}^{\prime}$. С другой стороны, $B^{\prime} \cap \Omega_{c}^{\prime}$ нигде не плотно в $\Omega_{c}^{\prime}$, поскольку $\operatorname{clos} B^{\prime}$ является компактным множеством. Полученное противоречие завершает доказательство.

СлЕДСТВИЕ 4.1. Пусть $f$ - структурно устойчивый $A$-диффеоморфизм замкнутого $n$-мерного многообразия $M^{n}, n \geqslant 3, u \Omega$ - ориентируемый растягивающийся аттрактор коразмерности один. Пусть $B-2$-связка, состоящая из двух неустойчивых многообразий $W^{u}(p) u W^{u}(q)$, где $p, q \in \Omega$ - ассочиированнье граничнье периодические точки, и $S_{p q}$ - характеристическая сфера, соответствующая $B$, которая, в свою очередь, есть обвединение связывающего иилиндра $C_{p q}$ и двух $(n-1)$-мерных шаров $D_{p} \subset W^{u}(p), D_{q} \subset W^{u}(q)$. Предположим, что $\Theta$ - базисное множество индекса $n-1$ такое, что пересечение $W^{u}(z) \cap C_{p q}, \quad z \in \Theta$, непусто. Тогда $\Theta$ - тривиальное базисное множество, а $z$-периодическая точка. Более того, $W^{u}(z) \cap C_{p q}$ состоит ровно из одной $(n-2)$-мерной сферы, скажем $S^{n-2}$, которая изотопна обеим сферам $S_{p}=\partial D_{p}$ и $S_{q}=\partial D_{q}$ в иилиндре $C_{p q}$. В частности, $S^{n-2}$ разбивает $C_{p q}$ на два иилиндра, гомеоморфные $\Sigma^{n-2} \times[0 ; 1]$.

СлЕДСТВИЕ 4.2. Пусть $f$ - структурно устойчивый А-диффеоморфизм замкнутого п-мерного многообразия $M^{n}, n \geqslant 3, u \Omega$ - ориентируемый растягивающийся аттрактор коразмерности один. Пусть $B-2$-связка, состоящая из двух неустойчивых многообразий $W^{u}(p)$ и $W^{u}(q)$, где $p, q \in \Omega$ - ассочиированные граничные периодические точки. Тогда $W_{\varnothing}^{s}(p)$ и $W_{\varnothing}^{s}(q)$ принадлежат $n$-мерным неустойчивым многообразиям источниковых периодических точек (возможно, совпадающих). 
ДокАЗАТЕльство. Предположим противное. Тогда $W^{u}(z) \cap\left(W_{\varnothing}^{s}(p) \cup\right.$ $\left.W_{\varnothing}^{s}(q)\right) \neq \varnothing$ для некоторой точки $z \in \Theta$, где базисное множество $\Theta$ имеет индекс $n-1$. Предположим для определенности, что $W^{u}(z) \cap W_{\varnothing}^{s}(p) \neq \varnothing$. Согласно теореме $4.2 \Theta$ - тривиальное базисное множество и $z$ - периодическая точка. Поэтому $W^{u}(z)$ пересекает $W_{\varnothing}^{s}(p)$ произвольно близко к $p$. Далее воспользуемся обозначениями следствия 4.1. Согласно $\lambda$-лемме $W^{u}(z)$ должна пересекать связывающий цилиндр $C_{p q}$ в бесконечном множестве компонент. Это противоречит следствию 4.1.

\section{$\S 5$. Гомотопический и топологический тип несущих многообразий}

Основной результат этого параграфа содержится в следующей теореме.

ТЕОРЕМА 5.1. Пусть $f$ - структурно устойчивый диффеоморфизм замкнутого $n$-мерного многообразия $M^{n}, \quad n \geqslant 3$, и $\Omega$ - ориентируемый растягивающийся аттрактор коразмерности один диффеоморфизма $f$. Тогда:

1) $M^{n}$ гомотопически әквивалентен $n$-мерному тору $T^{n}$; если $n \neq 4$, то $M^{n}$ гомеоморфен $T^{n}$;

2) $\Omega$ - единственное нетривиальное базисное множсество диффеоморфизмa $f$;

3) $f$ имеет, по крайней мере, одну источниковую периодическую точку.

ДоКАЗАТЕЛЬСТво. Возьмем произвольную связку $B$ базисного множества $\Omega$. Так как $\Omega$ ориентируемое, то $B$ является 2 -связкой, состоящей из неустойчивых многообразий $W^{u}(p)$ и $W^{u}(q)$, где $p, q$ - ассоциированные граничные периодические точки множества $\Omega$ [26]. Пусть $S_{p q}$ - характеристическая сфера, соответствуюшая $B$ и состояшая из двух $(n-1)$-мерных шаров $D_{p} \subset W^{u}(p), D_{q} \subset W^{u}(q)$ и связывающего $(n-1)$-мерного цилиндра $C_{p q} \subset W^{s}(\Omega)$. Согласно теореме 3.1 для доказательства гомотопической эквивалентности $M^{n}$ и $T^{n}$ покажем, что $S_{p q}$ ограничивает шар.

Можно считать, перейдя к некоторой итерации $f^{k}$, если необходимо, что обе точки $p$ и $q$ являются неподвижными. Тогда в силу следствия $4.2 W_{\varnothing}^{s}(p)$ и $W_{\varnothing}^{s}(q)$ принадлежат неустойчивым $n$-мерным многообразиям источниковых неподвижных точек (или, просто, истоков) $\alpha_{p}$ и $\alpha_{q}$ соответственно. Следовательно, $p$ и $q$ принадлежат достижимой гранище $\delta\left(W^{u}\left(\alpha_{p}\right)\right)$ и $\delta\left(W^{u}\left(\alpha_{q}\right)\right)$ соответственно. Согласно теореме о непрерывной зависимости неустойчивых многообразий от начальных условий $W^{u}(p)$ и $W^{u}(q)$ также принадлежат $\delta\left(W^{u}\left(\alpha_{p}\right)\right)$ и $\delta\left(W^{u}\left(\alpha_{q}\right)\right)$ соответственно. Следовательно, $W^{u}\left(\alpha_{p}\right)$ и $W^{u}\left(\alpha_{q}\right)$ необходимо пересекают цилиндр $C_{p q}$. Очевидно, оба пересечения $W^{u}\left(\alpha_{p}\right) \cap C_{p q}$ и $W^{u}\left(\alpha_{q}\right) \cap C_{p q}$ являются открытыми подмножествами цилиндра $C_{p q}$.

Отметим, что $C_{p q}$ может пересекать только $n$-мерные и $(n-1)$-мерные неустойчивые многообразия, поскольку $f$ структурно устойчивый и, следовательно, удовлетворяет сильному условию трансверсальности [51]. Если $C_{p q}$ не пересекается с $(n-1)$-мерными неустойчивыми многообразиями, то из связности $C_{p q}$ вытекает, что $W^{u}\left(\alpha_{p}\right)=W^{u}\left(\alpha_{q}\right)$ и $\alpha_{p}=\alpha_{q} \stackrel{\text { def }}{=} \alpha$. Поэтому $C_{p q} \subset W^{u}(\alpha)$.

В силу наличия локальной структуры произведения на $D_{p}$, для достаточно малого $\varepsilon>0$ множество

$$
N\left(D_{p}\right) \stackrel{\text { def }}{=} \bigcup_{x \in D_{p}} W_{\varepsilon}^{s}(x)
$$


является тотальным пространством тривиального расслоения

$$
\left(N\left(D_{p}\right), D_{p}, N\left(D_{p}\right) \rightarrow D_{p}\right) \cong\left(D_{p} \times(-1 ;+1), D_{p}, D_{p} \times(-1 ;+1), D_{p}\right)
$$

со слоем $W_{\varepsilon}^{s}(x)$ над каждой точкой $x \in D_{p}$. Ясно, что $D_{p}$ делит $N\left(D_{p}\right)$ на две области. Одну из них, скажем $N^{+}\left(D_{p}\right)$, пересекают как $C_{p q}$, так и $W_{\varnothing}^{s}(p)$. Отсюда и из включения $D_{p} \subset \delta\left(W^{u}(\alpha)\right)$ получаем

$$
\operatorname{clos} N^{+}\left(D_{p}\right) \subset D_{p} \cup W^{u}(\alpha) \subset \delta\left(W^{u}(\alpha)\right) \cup W^{u}(\alpha)
$$

Имея в виду естественный изоморфизм расслоений $N\left(D_{p}\right)$ и $D_{p} \times(-1 ;+1)$, положим

$D_{p, \varepsilon} \cong D_{p} \times\left\{\frac{1}{2}\right\} \subset N^{+}\left(D_{p}\right), \quad C_{p, \varepsilon}=\left(\bigcup_{x \in \partial D_{p}}(x, y)_{\varnothing, \varepsilon}^{s}\right) \cap C_{p q}=N^{+}\left(D_{p}\right) \cap C_{p q}$.

Из тривиальности расслоения $D_{p} \times(-1 ;+1)$ следует, что $C_{p, \varepsilon}$ является $(n-1)$-мерным цилиндром, гомеоморфным $C_{p q}$. Поэтому объединение $C_{p, \varepsilon} \cup$ $D_{p}$ является $(n-1)$-мерным шаром, принадлежашим характеристической сфере $S_{p q}$. Нетрудно также показать, что $D_{p, \varepsilon}, D_{p}$ и $C_{p, \varepsilon}$ ограничивают $n$-мерньй шар $B_{p} \subset W^{u}(\alpha)$.

Пусть $D_{q, \varepsilon}, C_{q, \varepsilon}$ - аналогичные $(n-1)$-мерные шар и подшилиндр соответственно, построенные в некоторой “трубчатой” области $N\left(D_{q}\right)$ вокруг $D_{p}$. Положим

$$
C_{p q, \varepsilon} \stackrel{\text { def }}{=} C_{p q}-\left(C_{p, \varepsilon} \cup C_{q, \varepsilon}\right)
$$

Тогда $S_{p q}^{\prime}=C_{p q, \varepsilon} \cup D_{p, \varepsilon} \cup D_{q, \varepsilon}$ является $(n-1)$-мерной сферой, изотопной $S_{p q}$ в $W^{u}(\alpha)$. Заметим, что $D_{p} \subset \delta\left(W^{u}(\alpha)\right)$ и $D_{q} \subset \delta\left(W^{u}(\alpha)\right)$ изотопны $D_{p, \varepsilon}$ и $D_{q, \varepsilon}$ в $B_{p} \subset W^{u}(\alpha)$ и $B_{q} \subset W^{u}(\alpha)$ соответственно.

Не уменьшая общности, можно считать, что $S_{p q}^{\prime}$ является цилиндрически вложенной сферой. Поэтому $S_{p q}^{\prime}$ ограничивает замкнутый $n$-мерньй шар, скажем $B_{p q}^{\prime}$, в $W^{u}(\alpha)$, поскольку $W^{u}(\alpha)$ гомеоморфно $\mathbb{R}^{n}$. Отсюда вытекает, что $S_{p q}$ также ограничивает замкнутый $n$-мерный шар $B_{p q}^{\prime} \cup B_{p} \cup B_{q}$ в $W^{u}(\alpha)$. Таким образом, мы доказали, что если $C_{p q}$ не пересекается с $(n-1)$-мерными неустойчивыми многообразиями, то $S_{p q}$ ограничивает $n$-мерньй замкнутый шар в $M^{n}$.

Предположим теперь, что $C_{p q}$ пересекает $(n-1)$-мерные неустойчивые многообразия. Согласно теореме 4.2 каждое из этих неустойчивых многообразий является неустойчивым многообразием некоторой седловой периодической точки, которую мы обозначим через $P_{i}$. Орбиты каждой такой точки $P_{i}$ образуют тривиальное базисное множество индекса $n-1$. Поэтому имеется только конечное число таких точек, так как число базисных множеств конечно. Итак, мы имеем конечное число периодических седловых точек $P_{1}, \ldots, P_{k}$ таких, что $W^{u}\left(P_{i}\right) \cap C_{p q} \neq \varnothing, 1 \leqslant i \leqslant k$.

Согласно следствию 4.1 для данной точки $P_{i}$ пересечение $W^{u}\left(P_{i}\right) \cap C_{p q}$ состоит ровно из одной $(n-2)$-мерной сферы, скажем $S_{i}^{n-2}$, которая изотопна $S_{p}=\partial D_{p}$ и $S_{q}=\partial D_{q}$ в цилиндре $C_{p q}$. В частности, $S_{i}^{n-2}$ разбивает $C_{p q}$ на два цилиндра, гомеоморфные $\Sigma^{n-2} \times[0 ; 1]$. Поэтому можно так перенумеровать точки $P_{i}$, чтобы 


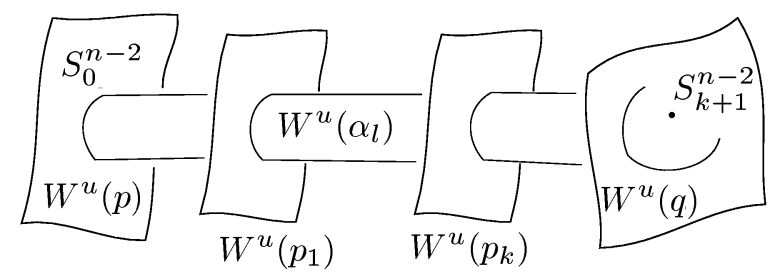

Рис. 16. Сферы $S_{i}^{n-2}=W^{u}\left(P_{i}\right) \cap C_{p q}$

соответствуюшие им сферы $S_{i}^{n-2}$ были упорядочены в цилиндре $C_{p q}$, начиная с $S_{0}^{n-2}=W^{u}(p) \cap \partial C_{p q}=\partial D_{p}$ до $S_{k+1}^{n-2}=W^{u}(q) \cap \partial C_{p q}=\partial D_{q}$.

Переобозначим также $\alpha_{p}$ и $\alpha_{q}$ на $\alpha_{0}$ и $\alpha_{k}$ соответственно (см. рис. 16).

Каждая сфера $S_{i}^{n-2}=W^{u}\left(P_{i}\right) \cap C_{p q}$ шилиндрически вложена в $W^{u}\left(P_{i}\right)$ и, следовательно, ограничивает замкнутьй $(n-1)$-мерньй шар $D_{i} \subset W^{u}\left(P_{i}\right)$, поскольку $W^{u}\left(P_{i}\right) \cong \mathbb{R}^{n-1}, i=1, \ldots, k$. Так как $S_{i}^{n-2}$ и $S_{i+1}^{n-2}$ изотопны в $C_{p q}$, то они ограничивают область $C_{i} \subset C_{p q}$, гомеоморфную открытому цилиндру $\Sigma^{n-2} \times(0 ; 1)$, $i=0, \ldots, k$. Поэтому объединение

$$
S_{i} \stackrel{\text { def }}{=} D_{i} \cup C_{i} \cup D_{i+1}
$$

является $(n-1)$-мерной сферой, цилиндрически вложенной в $M^{n}$. Согласно следствию 4.2 каждый цилиндр $C_{i}$ принадлежит $n$-мерному неустойчивому многообразию источниковой периодической точки, скажем $\alpha_{i}$. Перейдя к некоторой итерации $f$, если необходимо, мы можем считать, что все точки $p=P_{0}, P_{1}, \ldots, P_{k}$, $P_{k+1}=q, \alpha_{0}, \ldots, \alpha_{k}$ неподвижны.

Из теоремы о непрерывной зависимости инвариантных многообразий от начальных условий и из включений $C_{i} \subset W^{u}\left(\alpha_{i}\right), C_{i-1} \subset W^{u}\left(\alpha_{i-1}\right)$ вытекает, что

$$
D_{i} \subset \delta\left(W^{u}\left(\alpha_{i}\right)\right) \cap \delta\left(W^{u}\left(\alpha_{i-1}\right)\right), \quad i=1, \ldots, k .
$$

Поэтому $W^{s}\left(P_{i}\right) \cap W^{u}\left(\alpha_{i}\right) \neq \varnothing$ и $W^{s}\left(P_{i}\right) \cap W^{u}\left(\alpha_{i-1}\right) \neq \varnothing$. Заметим, что $P_{i}-$ седловая неподвижная точка индекса $n-1$. Следовательно, $W^{s}\left(P_{i}\right)$ - одномерная кривая, которая делится точкой $P_{i}$ на две кривые $W^{s-}\left(P_{i}\right)$ и $W^{s+}\left(P_{i}\right)$. Каждая из них не может пересекаться с неустойчивыми $(n-1)$-мерными многообразиями, поскольку в противном случае $C_{i-1}$ или $C_{i}$ также пересекались бы с неустойчивыми $(n-1)$-мерными многообразиями, что невозможно. Таким образом,

$$
W^{s-}\left(P_{i}\right) \cup W^{s+}\left(P_{i}\right) \subset W^{u}\left(\alpha_{i}\right) \cup W^{u}\left(\alpha_{i-1}\right) .
$$

Аналогично рассмотренному выше случаю $k=0$ покажем, что $(n-1)$-мерная сфера $S_{0}$ ограничивает $n$-мерный шар $B_{0} \subset W^{u}\left(\alpha_{0}\right)$. В силу наличия локальной структуры произведения на $\Omega$, для любой точки $x \in D_{0}-P_{0}$ дуга $\left[x, \varphi_{p q}(x)\right]_{\varnothing}^{s}$ входит в $B_{0}$. Эта дуга должна выйти из $B_{0}$, поскольку точка $\varphi_{p q}(x)$ лежит вне $B_{0}$ (напомним, что $D_{0}=D_{p}$ и $P_{0}=p$ ). Пусть $\varphi_{01}(x)$ - первая точка пересечения дуги $\left[x, \varphi_{p q}(x)\right]_{\varnothing}^{s}$ с $S_{0}=\partial B_{0}$ после $x$. В силу ориентируемости $\Omega, \varphi_{01}(x) \notin D_{0}$. Следовательно, $\varphi_{01}(x) \in D_{1}-P_{1}$. Так как устойчивые многообразия попарно не пересекаются, то отображение

$$
\varphi_{01}: D_{0}-P_{0} \rightarrow D_{1}-P_{1}
$$


взаимно однозначное. Отсюда вытекает, что обе кривые $W^{s-}\left(P_{1}\right)$ и $W^{s+}\left(P_{1}\right)$ не пересекают $D_{1}-P_{1}$. Поэтому существует ровно одна компонента множества $W^{s}\left(P_{1}\right)-P_{1}$, скажем $W^{s-}\left(P_{1}\right)$, которая лежит в $B_{0} \cap W^{u}\left(\alpha_{0}\right)$. Другая компонента $W^{s+}\left(P_{1}\right)$ лежит в $W^{u}\left(\alpha_{1}\right)$. В частности, $\alpha_{0} \neq \alpha_{1}$.

Продолжая рассуждать подобным образом, получаем, что каждая $(n-1)$-мерная сфера $S_{i}$ ограничивает $n$-мерный шар $B_{i} \subset W^{u}\left(\alpha_{i}\right)$, а точки $\alpha_{0}, \ldots, \alpha_{k}$ попарно различны. Следовательно, открытые шары int $B_{0}, \ldots$, int $B_{k}$ также попарно различны. Отсюда следует, что характеристическая сфера $S_{p q}$ ограничивает открытый $n$-мерный шар

$$
\bigcup_{i=1}^{k} \operatorname{int} B_{i} \bigcup_{i=1}^{k} \operatorname{int} D_{i} \stackrel{\text { def }}{=} B_{p q}^{n} .
$$

Согласно теореме 3.1 многообразие $M^{n}$ гомотопически эквивалентно $n$-мерному тору $T^{n}$.

В [46] доказано, что любое замкнутое $n$-мерное, $n \geqslant 5$, многообразие, гомотопически эквивалентное тору, гомеоморфно тору. Таким образом, для доказательства п. 1) осталось показать, что в случае $n=3$ многообразие $M^{3}$ гомеоморфно $T^{3}$.

Согласно лемме 2 из [65] $M^{3}$ неприводимо (т.е. любая цилиндрически вложенная 2-сфера $S^{2} \subset M^{3}$ ограничивает в $M^{3}$ шар), поскольку имеет универсальное накрьваюшее, гомеоморфиное $\mathbb{R}^{3}$. Теперь применим теорему Столлингса [67], которая утверждает, что если сушествует эпиморфизм $\pi_{1}\left(M^{3}\right)$ на $\mathbb{Z}$, ядро которого отлично от $\mathbb{Z}_{2}$, то $M^{3}$ является расслоением над окружностью $S^{1}$ со слоем, являющимся двумерной поверхностью $T$. Так как $\pi_{1}\left(M^{3}\right)$ изоморфна $\mathbb{Z}^{3}$, то такой эпиморфизм сушествует. Действительно, если $\gamma_{1}, \gamma_{2}, \gamma_{3}$ - образующие группы $\pi_{1}\left(M^{3}\right)$, то положим $\gamma_{1} \rightarrow 1$ и $\gamma_{2}, \gamma_{1} \rightarrow 0$, продолжив далее по линейности. В силу коммутативности $\pi_{1}\left(M^{3}\right)$, ядро данного эпиморфизма отлично от $\mathbb{Z}_{2}$.

Таким образом, $M^{3}$ является расслоением над $S^{1}$ со слоем $T$. Точная гомотопическая последовательность

$$
\pi_{3}\left(S^{1}\right) \rightarrow \pi_{2}(T) \rightarrow \pi_{2}\left(M^{3}\right) \rightarrow \pi_{2}\left(S^{1}\right) \rightarrow \pi_{1}(T) \rightarrow \pi_{1}\left(M^{3}\right) \rightarrow \pi_{1}\left(S^{1}\right) \rightarrow 0
$$

расслоения $M^{3} \rightarrow S^{1}$ принимает вид

$$
0 \rightarrow \pi_{2}(T) \rightarrow \pi_{2}\left(M^{3}\right) \rightarrow 0 \rightarrow \pi_{1}(T) \rightarrow \mathbb{Z}^{3} \rightarrow \mathbb{Z}^{1}
$$

Следовательно, $\pi_{2}(T)=\pi_{2}\left(M^{3}\right)=0$ и $\pi_{1}(T)$ мономорфно вкладывается в коммутативную групп $\mathbb{Z}^{3}$. Поэтому поверхность $T$ имеет коммутативную фундаментальную группу $\pi_{1}(T)$ и нулевую группу $\pi_{2}(T)$. Отсюда вытекает, что $T$ есть двумерный тор $T^{2}=S^{1} \times S^{1}$.

Теперь расслоение $M^{3}$ можно представить в виде произведения $T \times \mathbb{R}^{1}$, профакторизованному по следуюшему отношению эквивалентности: $(m, x+1) \in T \times \mathbb{R}^{1}$ отождествляется с $(f(m), x)$ для некоторого диффеоморфизма $f: T \rightarrow T$. В качестве образующих группы $\pi_{1}\left(M^{3}\right)$ можно взять элементы $a, b \in \pi_{1}(T)$ и $c$, удовлетворяющие соотношениям:

$$
a b=b a, \quad f_{*}(a)=c^{-1} a c, \quad f_{*}(b)=c^{-1} b c,
$$

где $f_{*}: \pi_{1}(T) \rightarrow \pi_{1}(T)$ - гомоморфизм, индуцированный диффеоморфизмом $f$ [43]. Так как $\pi_{1}(T)$ коммутативна, то $f_{*}(a)=a$ и $f_{*}(b)=b$. Следовательно, $f$ изотопен 
тождественному. Отсюда получаем гомеоморфность $M^{3}$ тору $T \times S^{1}=S^{1} \times S^{1} \times$ $S^{1}$. Это завершает доказательство п. 1).

Утверждение 2) следует из утверждения 1) и теоремы 3.1. Для доказательства утверждения 3) заметим, что любой ориентируемый растягивающийся аттрактор коразмерности один имеет, по крайней мере, одну 2-связку $B$. Согласно следствию 4.2 внутри характеристической сферы, соответствуюшей связке $B$, лежит периодическая точка, принадлежащая тривиальному репеллеру. Это завершает доказательство теоремы.

Следующие утверждения непосредственно вытекают из доказательства вышеприведенной теоремы.

СледСТВИЕ 5.1. Пусть $f$ - структурно устойчивый диффеоморфизм $n$-мерного замкнутого многообразия $M^{n}, n \geqslant 3$, неблуждающее множество которого содержит ориентируемый растягивающийся аттрактор $\Omega$ коразмерности один. Тогда спектральное разложсение диффеоморфизма $f$ состоит из $\Omega$, конечного (возможно, пустого) множества периодических орбит индекса $n-1$ и конечного непустого множества периодических орбит индекса $n$.

СледСТВИЕ 5.2. Пусть $f$ - структурно устойчивый диффеоморфизм $n$-мерного замкнутого многообразия $M^{n}, n \geqslant 3$, имеющий ориентируемый растягивающийся аттрактор $\Omega$ коразмерности один. Пусть $S_{p q}$ - характеристическая сфера, соответствующая 2-связке $B=W^{u}(p) \cup W^{u}(q)$ аттрактора $\Omega$, где $p, q \in \Omega$ - ассочиированнье граничнье периодические точки. Тогда $S_{p q}$ ограничивает п-мерный шар, содержащий $k_{0}+1$ источниковых периодических точек $\alpha_{1}, \ldots, \alpha_{k_{0}+1}, k_{0} \geqslant 0$, индекса $n$ и $k \geqslant 0$ седловых периодических точек $P_{1}, \ldots, P_{k}$ индекса $n-1$. Более того, выполняются следующие условия:

1) $k=k_{0}$

2) обгединение

$$
a_{p q}=\{p\} \cup W_{\varnothing}^{s}(p) \cup \alpha_{1} \cup W^{s}\left(P_{1}\right) \cup P_{1} \cup \alpha_{2} \cup \cdots \cup P_{k} \cup \alpha_{k+1} \cup W_{\varnothing}^{s}(q) \cup\{q\}
$$

является компактной дугой без самопересечений с концевыми точками $p, q$;

3) на дуге $a_{p q}$ источниковые периодические точки индекса $n$ чередуются с седловыми периодическими точками индекса $n-1$, включая $p$ u $q$ (см. puc. 17).

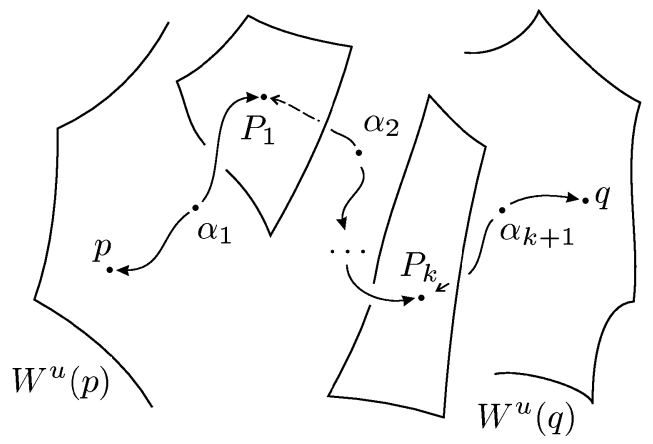

Рис. 17. Дуга $a_{p q}$ 
СЛЕДСТВИЕ 5.3. Пусть $f-A$-диффеоморфизм $n$-мерного замкнутого многообразия $M^{n}, \quad n \geqslant 3$, и пусть $\Omega$ - ориентируемый растягивающийся (сжимающийся) аттрактор (репеллер) коразмерности один диффеоморфизма $f$. Тогда следующие условия влекут структурную неустойчивость диффеоморфизма $f$ :

1) диффеоморфизм $f$ имеет нетривиальное базисное множество, отличное от $\Omega$;

2) многообразие $M^{n}$ не гомеоморфно п-мерному тору.

\section{§6. Топологическая классификация структурно устойчивых диффеоморфизмов с базисными множествами коразмерности один на торе}

После теоремы 5.1 естественным шагом по пути классификации структурно устойчивых диффеоморфизмов с базисньми множествами коразмерности один является классификация структурно устойчивых диффеоморфизмов с растягивающимися аттракторами или сжимающимися репеллерами коразмерности один на торе $T^{n}, n \geqslant 3$. Пусть $f: T^{n} \rightarrow T^{n}$ - такой диффеоморфизм. Для определенности будем считать, что $f$ имеет растягиваюшийся аттрактор $\Omega$ коразмерности один. Обозначим через

$$
f_{*}: H_{1}\left(T^{n}, \mathbb{R}^{n}\right) \rightarrow H_{1}\left(T^{n}, \mathbb{R}^{n}\right)
$$

автоморфизм одномерной группы гомологий $H_{1}\left(T^{n}, \mathbb{R}^{n}\right) \simeq \mathbb{R}^{n}$ тора $T^{n}$, индуцированный диффеоморфизмом $f$. Автоморфизм $f_{*}$ назьвается гиперболическим, если он не имеет собственных значений, по модулю равных единище. Автоморфиизм называется гиперболическим автоморфизмом коразмерности один, если он имеет единственное собственное число, либо меньшее, либо большее единицы по абсолютной величине, а остальные собственные числа лежат соответственно либо вне единичной окружности комплексной плоскости, либо внутри. Следующая теорема была доказана в [11] и [12].

ТЕОРема 6.1. Пусть $f: T^{n} \rightarrow T^{n}-A$-диффеоморфизм $n$-мерного тора $T^{n}$, $n \geqslant 3$, имеющий ориентируемыи растягивающийся аттрактор $\Omega$ коразмерности один. Тогда $f_{*}$ - гиперболический автоморфизм коразмерности один.

Следуя [39], будем называть диффеоморфизм $f: M \rightarrow M \pi_{1}$-диффеоморфизмом, если для любого гомеоморфизма $g: K \rightarrow K$ компактного $C W$-комплекса $K$ на себя и непрерывного отображения $h: K \rightarrow M$, для которых выполняется соотношение $f_{*} \circ h_{*}=h_{*} \circ g_{*}$, сушествует единственное отображение $h^{\prime}: K \rightarrow M$, переводящее базисную точку на $K$ в базисную точку на $M$, гомотопное $h$ и такое, что $f \circ h^{\prime}=h^{\prime} \circ g$.

Согласно теореме 6.1 существует алгебраический автоморфизм $A(f): T^{n} \rightarrow T^{n}$ с $f_{*}=A(f)_{*}$, который является гиперболическим. В силу предложения 2.1 из [39] гиперболический автоморфизм тора является $\pi_{1}$-диффеоморфизмом. Поэтому существует непрерывное отображение $h: T^{n} \rightarrow T^{n}$, гомотопное тождественному, такое, что $h \circ f=A(f) \circ h$. Положим

$$
P(f, h)=\left\{x \in T^{n} \mid h^{-1}(x) \text { содержит более одной точки }\right\} .
$$


ЛЕмма 6.1. Пусть $f: T^{n} \rightarrow T^{n}-A$-диффеоморфизм $n$-мерного тора $T^{n}$, $n \geqslant 3$, имеющий ориентируемый растягивающийся аттрактор $\Omega$ коразмерности один, и пусть $h: T^{n} \rightarrow T^{n}$ - непрерывное отображение, гомотопное тождественному, такое, что $h \circ f=A(f) \circ h$. Тогда $h$ удовлетворяет следуюшим условиям:

a) $h(\Omega)=T^{n}$;

б) пусть $\left\{p_{i}, q_{i}\right\}_{i=1}^{k}$ - семейство пар ассочичрованных граничных периодических точек диффеоморфизма $f ;$ тогда $h\left(p_{i}\right)=h\left(q_{i}\right)$ является периодической точкой автоморфизма $A(f)$ для каждого $i=1, \ldots, k$;

в) $h\left(W^{u}\left(p_{i}\right)\right)=h\left(W^{u}\left(q_{i}\right)\right), \quad i=1, \ldots, k$;

г) $P(f, h)=\bigcup_{i=1}^{k} h\left(W^{u}\left(p_{i}\right)\right)$;

д) пусть $K_{i}$ - компонента множества $T^{n}-\Omega$; тогда $h\left(K_{i}\right)$ есть неустойчивое многообразие $W^{u}\left(h\left(p_{i}\right)\right)=W^{u}\left(h\left(q_{i}\right)\right)$ автоморфизма $A(f)$, где $p_{i}$, $q_{i}$ - ассочиированные граничные периодические точки такие, что $\delta\left(K_{i}\right)=$ $W^{u}\left(p_{i}\right) \cup W^{u}\left(p_{i}\right)$, более того, $h\left(K_{i} \cup \delta\left(K_{i}\right)\right)=W^{u}\left(h\left(p_{i}\right)\right)$;

е) пусть $\breve{\Omega} \subset \Omega$ - оббединение неустойчивых многообразий, не содержащих граничных периодических точек; тогда ограничение $h \mid \breve{\Omega}$ является гомеоморфизмом на свой образ.

ДоКАЗАТЕЛЬСТво аналогично доказательствам теоремы 2 из [11] и леммы 4 из [12]. Мы его опускаем.

СлЕДСТВИЕ 6.1. Пусть $f: T^{n} \rightarrow T^{n}$ - структурно устойчивый диффеоморфизм $n$-мерного, $n \geqslant 3$, тора $T^{n}$, имеющий ориентируемый растягивающийся аттрактор $\Omega$ коразмерности один. Тогда $A(f)$ является алгебрачческим автоморфизмом Аносова коразмерности один.

ДОКАЗАТЕЛЬСТВО следует из соотношения $h \circ f=A(f) \circ h$ и леммы 6.1.

Пусть $\Omega$ - ориентируемый растягиваюшийся аттрактор коразмерности один структурно устойчивого диффеоморфизма $f$, и пусть $S_{p q}$ - характеристическая сфера, соответствуюшая 2-связке $B=W^{u}(p) \cup W^{u}(q)$ аттрактора $\Omega$, где $p, q \in \Omega$ ассоциированные граничные периодические точки. Согласно следствию 5.2 внутри сферы $S_{p q}$ лежит $d \geqslant 1$ периодических точек индекса $n$ и $d-1 \geqslant 0$ периодических точек индекса $n-1$. Положим $d(p, q)=d$. Если $\Omega$ - репеллер, то через $d(p, q)$ обозначим число периодических точек индекса 0 , лежащих внутри $S_{p q}$. В обоих случаях число $d(p, q)$ корректно определено, так как не зависит от выбора характеристической сферы $S_{p q}$. Нетрудно видеть, что точки $f^{j}(p), f^{j}(q)$ являются ассоциированными граничными и периодическими, а число периодических точек одного индекса внутри сфер $S_{p q}$ и $f^{j}\left(S_{p q}\right)=S_{f^{j}(p), f^{j}(q)}$ одинаковое для любого $j \in \mathbb{Z}$. Поэтому можно приписать число $d(O(p, q)) \stackrel{\text { def }}{=} d(p, q)$ объединению $O(p, q)=O(p) \cup O(q)$ орбит точек $p, q$.

Граничные периодические точки разбиваются на пары $\left\{O\left(p_{i}, q_{i}\right)\right\}_{i=1}^{k}$ орбит ассоциированных граничных точек. Пусть $\left\{d\left(O\left(p_{i}, q_{i}\right)\right)\right\}_{i=1}^{k}-$ соответствуюшие им определенные выше числа, указывающие на количество периодических точек индекса $n$ (если $\Omega$ - аттрактор) или индекса 0 (если $\Omega$ - репеллер) в соответствующих характеристических сферах. Согласно лемме $6.1 h\left(O\left(p_{i}\right)\right)=h\left(O\left(q_{i}\right)\right)$ является периодической орбитой автоморфизма $A(f)$. Припишем каждой орбите $h\left(O\left(p_{i}\right)\right)=h\left(O\left(q_{i}\right)\right)$ число $d\left(O\left(p_{i}, q_{i}\right)\right)$. Семейство $\left\{h\left(O\left(p_{i}\right)\right), d\left(O\left(p_{i}, q_{i}\right)\right)\right\}_{i=1}^{k}$ называется $d$-сигнатурой диффеоморфизма $f$ и обозначается через $\mathscr{D}(f, h)$. 
Пусть $A$ - гиперболический автоморфизм коразмерности один тора $T^{n}$, и пусть $\left\{O_{j}\right\}_{j=1}^{r}-$ конечный набор периодических орбит $O_{j}$ автоморфизма $A$. Каждой орбите $O_{j}$ припишем произвольным образом натуральное число $d_{j} \in \mathbb{N}$. Семейство $\left\{O_{j}, d_{j}\right\}_{j=1}^{r}$ называется допустимой $d$-сигнатурой автоморфизма $A$. Согласно теореме $5.1 d$-сигнатура структурно устойчивого диффеоморфизма $f$ является допустимой.

Пусть $\left\{O_{j}^{1}, d_{j}^{1}\right\}_{j=1}^{r_{1}}, \quad\left\{O_{j}^{2}, d_{j}^{2}\right\}_{j=1}^{r_{2}}-$ допустимые $d$-сигнатуры гиперболических автоморфизмов $A_{1}$ и $A_{2}$ соответственно. Эти сигнатуры называются әквивалентнымми, если существует линейньй диффеоморфизм (т.е. композиция автоморфизма и сдвига) $\psi: T^{n} \rightarrow T^{n}$ такой, что $\psi\left(\bigcup_{j=1}^{r_{1}}\left(O_{j}^{1}\right)\right)=\bigcup_{j=1}^{r_{2}}\left(O_{j}^{2}\right), d\left(\psi\left(O_{j}\right)\right)=d\left(O_{j}\right)$ для всех $1 \leqslant j \leqslant r_{1}$ и выполняется соотношение $\psi \circ A_{1}=A_{2} \circ \psi$. Непосредственно из определения следует, что $r_{1}=r_{2}$.

Следуюшая теорема решает задачу топологической сопряженности в классе структурно устойчивых диффеоморфизмов на торе $T^{n}, n \geqslant 3$, имеющих ориентируемые растягиваюшиеся аттракторы или сжимаюшиеся репеллеры коразмерности один. Она показывает, что $d$-сигнатура является полным инвариантом сопряженности в данном классе диффеоморфизмов.

Teорема 6.2. Пусть $f_{1}, f_{2}: T^{n} \rightarrow T^{n}$ - структурно устойчивые диффеоморфизмы, имеющие ориентируемые растягивающиеся аттракторы коразмерности один $\Omega_{1}$ и $\Omega_{2}$ соответственно. Диффеоморфизмы $f_{1}, f_{2}$ сопряженьи тогда и только тогда, когда их d-сигнатурь $\mathscr{D}\left(f_{1}, h_{1}\right), \mathscr{D}\left(f_{2}, h_{2}\right)$ әквивалентны, где $h_{i}: T^{n} \rightarrow T^{n}, i=1,2,-$ гомотопные тождественному непрерывные отображения такие, что $h_{i} \circ f_{i}=A\left(f_{i}\right) \circ h_{i}$.

ДоКАЗАТЕЛЬСТво проводится по схеме доказательств теоремы 3 из [11] и теоремы 2 из [12], где рассматривалась сопряженность диффеоморфизмов в окрестностях базисных множеств без предположения о структурной устойчивости (см. также [26, теорема 2.5$]$ и [ $[17$, теорема 4]). В данном случае лемма 6.1 и следствие 5.2 позволяют получить доказательство сопряженности не только в окрестности базисных множеств, но и на всем торе.

Следуюшая теорема решает задачу реализации в классе структурно устойчивых диффеоморфизмов на торе $T^{n}, n \geqslant 3$, имеюших ориентируемые базисные множества коразмерности один (растягивающиеся аттракторы или сжимающиеся репеллеры). Именно, для каждого допустимого топологического инварианта ( $d$-сигнатуры) строится структурно устойчивый диффеоморфизм с данным инвариантом.

Теорема 6.3. Пусть А: $T^{n} \rightarrow T^{n}$ - гиперболический автоморфизм с неустойчивым расслоением коразмерности один в каждом слое касательного расслоения тора $T^{n}, \quad n \geqslant 3$ (это означает, что устойчивые многообразия всех точек одномерные). Для любой допустимой $d$-сигнатуры $\left\{O_{j}, d_{j}\right\}_{j=1}^{r}$ автоморфизма А существует структурно устойчивый диффеоморфизм $f: T^{n} \rightarrow$ $T^{n}$, имеющий ориентируемый растягивающийся аттрактор коразмерности один такой, что $\mathscr{D}(f, h)=\left\{O_{j}, d_{j}\right\}_{j=1}^{r}$, әде $f_{*}=A_{*} u h: T^{n} \rightarrow T^{n}$ - непрерывное гомотопное тождественному отображение, удовлетворяющее соотношению $h \circ f=A \circ h$. 
ДОКАЗАТЕЛЬСТВО проведем, используя так назьваемую "хирургическую операцию", описанную Смейлом [66] и Вильямсом [68] при построении $D A$-диффеоморфизма с одномерным базисным множеством на 2-мерном торе. Строгое изложение конструкции имеется в [48] (для 2-мерного тора), в [40] (для 3-мерного тора) и в [26] (для $n$-мерного, $n \geqslant 2$, тора). Согласно теореме 2.3 из [26] сушествует структурно устойчивый $D A$-диффеоморфизм $f^{\prime}: T^{n} \rightarrow T^{n}$, имеющий единственный ориентируемый растягиваюшийся аттрактор коразмерности один $\Omega^{\prime}$ с $d$-сигнатурой $\left\{O_{j}, 1\right\}_{j=1}^{r}$. Затем, учитывая следствие 5.2 , можно модифицировать $f^{\prime}$ внутри характеристических сфер таким образом, чтобы получить требуемый диффеоморфизм $f$ с $d$-сигнатурой $\left\{O_{j}, d_{j}\right\}_{j=1}^{r}$ и базисным множеством $\Omega=\Omega^{\prime}$.

\section{Список литературы}

1. Аносов Д. В. Геодезические потоки на замкнутых римановых многообразиях отрицательной кривизны // Тр. МИАН СССР. 1967. Т. 90.

2. Аносов Д. В. Об одном классе инвариантных множеств гладких динамических систем // Тр. пятой международной конференции по нелинейным колебаниям. Т. 2. Качественные методы. Киев: Ин-т математики АН УССР, 1970. С. 39-45.

3. Аносов Д. В. Грубые системы // Тр. МИАН СССР. 1985. Т. 169. С. 59-93.

4. Аносов Д. В., Солодов В.В. Гиперболические множества // Современные проблемы математики. Фундаментальные направления / Ред. Д. В. Аносов. Т. 66. Динамические системы-9. М.: ВИНИТИ, 1991. С. 12-99.

5. Арансон C.X., Гринес В.З. Каскады на поверхностях // Современные проблемы математики. Фундаментальные направления / Ред. Д. В. Аносов. Т. 66. Динамические системы-9. М.: ВИНИТИ, 1991. С. 148-187.

6. Арансон C.X., Жужома Е. В. О структуре квазиминимальных множеств слоений на поверхностях // Матем. сб. 1994. Т. 185. С. 31-62.

7. Гринес В.З. О топологической сопряженности диффеоморфизмов двумерного многообразия на одномерных базисных множествах // УМН. 1974. Т. 29. №6. С. 163-164.

8. Гринес В.З. О топологической сопряженности диффеоморфизмов двумерного многообразия на одномерных ориентируемых базисных множествах. 1 // Тр. ММО. 1975. Т. 32. C. $35-60$.

9. Гринес В.З. О топологической сопряженности диффеоморфизмов двумерного многообразия на одномерных ориентируемых базисных множествах. 2 // Тр. ММО. 1977. Т. 34. C. $243-252$.

10. Гринес В.З. О топологической классификации структурно устойчивых диффеоморфизмов поверхностей с одномерными аттракторами и репеллерами // Матем. сб. 1997. T. 188 . C. $57-94$.

11. Гринес В.З., Жужсма Е.В. О топологической классификации ориентируемых аттракторов на $n$-мерном торе // УМН. 1979. Т. 34. № 4. С. 185-186.

12. Гринес B.З., Жужсма E.B. Необходимые и достаточные условия топологической эквивалентности ориентируемых аттракторов на $n$-мерном торе // Дифференциальные и интегральные уравнения: Сб. научных трудов. Горький: Изд-во Горьк. гос. ун-та, 1981. C. 89-93.

13. Гринес В.З., Калай X.X. Диффеоморфизмы двумерных многообразий с просторно расположенными базисными множествами // УМН. 1985. Т. 40. № 1. С. 189-190.

14. Гринес В.З., Калай X. Х. О топологической эквивалентности диффеоморфизмов с нетривиальными базисными множествами на двумерных многообразиях // Методы качественной теории дифференциальных уравнений / Ред. Е.А. Леонтович-Андронова. Горький: Из-во Горьк. гос. ун-та, 1988. С. 40-49.

15. Гринес В.З., Калай X. X. Топологическая классификация базисных множеств без пар сопряженных точек $A$-диффеоморфизмов на поверхностях // Деп. ВИНИТИ 10.02.88. 1137 - В88. Горький, 1988. С. 1-95.

16. Жиров А. Ю. Гиперболические аттракторы диффеоморфизмов ориентируемых поверхностей. Ч. 1. Кодирование, классификация и накрытия // Матем. сб. 1994. Т. 185. №6. 
С. 3-50; Ч. 2. Перечисление и применение к псевдоаносовским диффеоморфизмам // Матем. сб. 1994. Т. 185. №9. С. 29-80; Ч. 3. Алгоритм классификации // Матем. сб. 1995. Т. 186. № 9. С. 59-82.

17. Жужсома Е.В. Ориентируемые базисные множества коразмерности $1 / /$ Изв. вузов. Математика. 1982. Т. 5. С. 16-21.

18. Келдыш Л.В. Топологические вложения в евклидово пространство // Тр. МИАН CCCP. 1966. T. 81.

19. Нитецки З. Введение в дифференциальную динамику. М.: Мир, 1975.

20. Новиков С. П. Топология слоений // Тр. ММО. 1965. Т. 14. С. 248-278.

21. Палис Ж., Ди Мелу В. Геометрическая теория динамических систем. Введение. М.: Мир, 1986.

22. Плькин Р. В. О топологии базисных множеств диффеоморфизмов Смейла // Матем. сб. 1971. T. 84. № 9. C. 301-312.

23. Пльљин Р. В. Источники и стоки $A$-диффеоморфизмов поверхностей // Матем. сб. 1974. T. 94. № 6. C. 243-264.

24. Плькин Р. В. О существовании притягивающих (отталкивающих) периодических точек $A$-диффеоморфизмов проективной плоскости и бутылки Клейна // УМН. 1977. Т. 32. №3. C. 179 .

25. Плькин Р. В. О гиперболических аттракторах диффеоморфизмов // УМН. 1980. Т. 35. № 3. C. $94-104$.

26. Плькин Р. В. О геометрии гиперболических аттракторов гладких каскадов // УМН. 1984. T. 39. № 6. C. 75-113.

27. Плькин Р. В., Сатаев Е. А., Шлячков С.В. Странные аттракторы // Современные проблемы математики. Фундаментальные направления / Ред. Д.В. Аносов. Т. 66. Динамические системы-9. М.: ВИНИТИ, 1991. С. 100-148.

28. Постников M. М. Лекции по алгебраической топологии. Основы теории гомотопий. М.: Наука, 1984.

29. Рохлин В. А., Фукс Д. Б. Начальный курс топологии. Геометрические главы. М.: Наука, 1977.

30. Стинрод Н. Топология косых произведений. М.: ИЛ, 1953.

31. Тамура И. Топология слоений. М.: Мир, 1979.

32. Хирш М. Дифференциальная топология. М.: Мир, 1979.

33. Aranson S., Belitsky G., Zhuzhoma E. Introduction to Qualitative Theory of Dynamical Systems on Closed Surfaces. Translations of Math. Monographs // Amer. Math. Soc. 1996. V. 153. P. 331.

34. Bonatti Ch., Langevin R. Difféomorphismes de Smale des surfaces // Astérisque. 1998. V. 250.

35. Bowen R. Periodic points and measures for axiom A-diffeomorfisms // Transactions of the American. Math. Soc. 1971. V. 154. P. 337-397.

36. Brown M. A proof of generalized Shoenflies theorem // Bull. Amer. Math. Soc. 1960. V. 66. № 2. P. 74-76.

37. Brown M., Gluck H. Stable structure on manifolds // Ann. of Math. 1964. V. 79. № 1. P. $1-58$.

38. Farrell F., Jones L. New attractors in hyperbolic dynamics // J. Diff. Geom. 1980. V. 15. P. 107-133.

39. Френкс Джс. Диффеоморфизмы Аносова // Гладкие динамические системы / Ред. Д. В. Аносов. М.: Мир, 1977. С. 32-86.

40. Franks J., Robinson C. A quasi-Anosov diffeomorphism that is not Anosov // Trans. AMS. 1976. V. 223. P. 267-278.

41. Guckenheimer J. Endomorphisms of Riemann sphere // Global Analisys. Proc. Symp. in Pure Math. AMS. 1970. V. 14. P. 95-123.

42. Hadamard $J$. Sur l'iteration et les solutions asymptotiques des équations différentielles // Bull. Soc. Math. France. 1901. V. 29. P. 224-228.

43. Hempel J. 3-manifolds // Annals Math. Studies. 1976. V. 86. P. 196. 
44. Hirch M., Pugh C. Stable manifolds and hyperbolic sets // Global Analysis. Proc. Symp. Amer. Math. Soc. 1970. V. 14. P. 133-163.

45. Hirch M., Palis J., Pugh C., Shub M. Neighborhoods of hyperbolic sets // Invent. Math. 1970. V. 9. P. 121-134.

46. Hsiang W. C., Wall C. T. C. On homotopy tori. II // Bull. London Math. Soc. 1969. V. 1. P. 341-342.

47. Jones L. Locally strange hyperbolic sets // Trans. Amer. Math. Soc. 1983. V. 275. № 1. P. 153-162.

48. Katok A., Hasselblatt B. Introduction to the Modern Theory of Dynamical Systems // Encyclopedia of Math. and its Appl. Cambridge: Cambridge Univ. Press, 1994.

49. Kirby R., Siebenmann L. On the triangulation of manifolds and Hauptvermutung // Bull. Amer. Math. Soc. 1979. V. 75. № 4. P. 742-749.

50. Kollmer H. On hyperbolic attractors of codimension one // Lect. Notes Math. 1977. V. 597. P. 330-334.

51. Mañé R. A proof of $C^{1}$ stability conjecture // Publ. Math. IHES. 1988. V. 66. P. 161-210.

52. Manning A. There are no new Anosov diffeomorphisms on tori // Amer. J. of Math. 1974. V. 96. P. $422-429$.

53. Mazur B. On embedding of spheres // Bull. Amer. Math. Soc. 1959. V. 65. P. 59-65.

54. Morgan J. W., Shalen P. Valuations, trees, and degenerations of hyperbolic structure, II: measured laminations in 3-manifolds // Annals of Math. 1988. V. 127. P. 403-465.

55. Newhouse S. E. On codimension one Anosov diffeomorphisms // Amer. J. Math. 1970. V. 92. № 3. P. 761-770.

56. Palis J. On Morse-Smale dynamical systems // Topology. 1969. V. 8. № 4. P. 385-404.

57. Perron $O$. Über Stabilität und asymptotisches Verhalten der Integrale von Differentialgleichungs systemen // Math. Z. 1928. V. 29. P. 129-160.

58. Perron O. Die Stabilitätsfrage bei Differentialgleichungen // Math. Z. 1930. V. 32. P. 703-728.

59. Plante J. On the existence of exceptional minimal sets in foliations of codimension one // J. Diff. Equat. 1974. V. 15. P. 178-194.

60. Plante J. The homology class of an expanded invariant manifolds // Lect. Notes Math. 1975. V. 468. P. 251-256.

61. Reeb G. Sur certaines propriétés topologiques des variétés feuilletées // Actual. Sci. Ind. № 1183. Paris: Hermann, 1952.

62. Robinson C. Structural stability of $C^{1}$ diffeomorphisms // J. Diff. Equat. 1976. V. 22. № 1. P. 28-73.

63. Robinson C. Dynamical Systems: stability, symbolic dynamics, and chaos // Studies in Adv. Math., Sec. edition, Boca Raton, FL. CRC Press, 1999. P. 506.

64. Robinson R. C., Williams R.F. Finite stability is not generic // Dynamical Systems: Proc. Symp. (Brazil, 1971). N. Y.-London: Academic Press, 1973. P. 451-462.

65. Rosenberg H. Foliations by planes // Topology. 1968. V. 7. P. 131-138.

66. Smale S. Differentiable dynamical systems // Bull. Amer. Math. Soc. 1967. V. 73. № 1. Р. 741-817; Смейл C. Дифференцируемые динамические системы // УМН. 1970. Т. 25. № 1(151). C. 113-185.

67. Stallings J. On fibering certain 3-manifolds // Topology of 3-manifolds. Proc. Univ. of Georgia Institute. N.J.: Printice-Hall, 1962. P. 95-100.

68. Williams R. F. The DA-maps of Smale and structural stability // Proc. Berk. Symp. 1970. V. 14. P. 329-334.

69. Williams R. Expanding attractors // Publ. Math. I.H.E.S. 1974. V. 43. P. 169-203. 